Supporting Information (SI)

\title{
Catch-Release System for Dosing and Recycling Silver(I) Catalyst with Status of Catalytic Activity Reported by Fluorescence
}

\author{
Indrajit Paul, ${ }^{\mathrm{a}}$ Nikita Mittal, ${ }^{\mathrm{a}}$ Soumen De, ${ }^{\mathrm{a}}$ Michael Bolte, ${ }^{\mathrm{b}}$ Michael Schmittel*,a \\ ${ }^{\text {a }}$ Center of Micro and Nanochemistry and Engineering, Organische Chemie I, Universität Siegen, Adolf- \\ Reichwein Str. 2, D-57068 Siegen, Germany. \\ ${ }^{\mathrm{b}}$ Institut für Anorganische und Analytische Chemie, Goethe-Universität Frankfurt, Max-von-Laue-Strasse \\ 7, D-60438 Frankfurt (Main), Germany. \\ E-mail: schmittel@,chemie.uni-siegen.de
}

Fax: (+49) 271-740-3270

\section{$\underline{\text { Table of Contents }}$}

$\begin{array}{lll}\text { 1. Synthesis } & \text { S2-S4 }\end{array}$

2. Characterization and metal complexes of nanoswitch $1 \quad$ S4-S6

$\begin{array}{lll}3 . & \text { Synthesis of ligand } 2 & \text { S6-S10 }\end{array}$

4. Synthesis and characterization of substrate for the catalysis S11-S12

5. Synthesis and characterization of Model complexes: S12-S14

$\begin{array}{ll}\text { 6. Model studies } & \text { S14-S15 }\end{array}$

7. NMR spectra: ${ }^{1} \mathrm{H}$ NMR, ${ }^{13} \mathrm{C}$ NMR, ${ }^{1} \mathrm{H}-{ }^{1} \mathrm{H}$ COSY S15-S29

$\begin{array}{ll}\text { 8. Catalytic Experiments } & \text { S29-S38 }\end{array}$

9. ESI-MS Spectra S39-S45

10. UV-Vis and Fluorescence Data S46-S52

$\begin{array}{ll}\text { 11. X-ray crystallography } & \text { S52-S53 }\end{array}$

12. References $\quad$ S54 


\section{Synthesis}

\subsection{General Remarks}

All solvents were dried by distillation prior to use whereas commercial reagents $(\mathbf{3}, \mathbf{8}, \mathbf{9}, 16$ hexacyclen) were used without any further purification. ${ }^{1} \mathrm{H}$ and ${ }^{13} \mathrm{C}$ NMR spectra were recorded on a Bruker Avance (400 MHz) spectrometer using a deuterated solvent as the lock and residual protiated solvent as internal reference $\left(\mathrm{CDCl}_{3}: \delta_{\mathrm{H}} 7.26 \mathrm{ppm}, \delta_{\mathrm{C}} 77.0 \mathrm{ppm} ; \mathrm{CD}_{2} \mathrm{Cl}_{2}: \delta_{\mathrm{H}} 5.32 \mathrm{ppm}\right.$, $\delta_{\mathrm{C}} 53.8 \mathrm{ppm}$, THF-d $\left.8: \delta_{\mathrm{H}} 1.72 \mathrm{ppm}, 3.58 \mathrm{ppm}, \delta_{\mathrm{C}} 25.3 \mathrm{ppm}, 67.2 \mathrm{ppm}\right)$. The following abbreviations were used to define NMR peak patterns: $\mathrm{s}=$ singlet, $\mathrm{d}=$ doublet, $\mathrm{t}=$ triplet, $\mathrm{dd}=$ doublet of doublets, ddd $=$ doublet of doublets of doublets, $\mathrm{td}=$ triplet of doublets, $\mathrm{br}=$ broad, $\mathrm{m}=$ multiplet. The coupling constants are given in Hertz $(\mathrm{Hz})$ and, wherever possible, assignment of protons is made. The carbons in the molecular skeletons were not necessarily numbered following the IUPAC nomenclature rules; numeration was exclusively done for assigning NMR signals. All electrospray ionization (ESI-MS) spectra were recorded on a Thermo-Quest LCQ deca and the theoretical isotopic distributions of the mass signals were calculated using IsoPro 3.0 software. Melting points of compounds were measured on a BÜCHI 510 instrument and are not corrected. Infrared spectra were recorded on a Varian 1000 FT-IR instrument. Elemental analysis was performed using the EA-3000 CHNS analyzer. UV-vis spectra were recorded on Cary Win 50 (298 K) and Cary Win 600 (298 K) spectrometer. Binding constants were determined through UV-vis titrations assuming a 1:1 binding scheme or with SPECFIT/32TM global analysis system from Spectrum Software Associates (Marlborough, MA). Column chromatography was performed either on silica gel (60-400 mesh) or neutral alumina (Fluka, 0.05-0.15 mm, Brockmann Activity 1). Merck silica gel (60 F254) or neutral alumina (150 F254) sheets were used for thin layer chromatography (TLC). Metal complexes were prepared directly in the NMR tube using $\mathrm{CD}_{2} \mathrm{Cl}_{2}$ and $\mathrm{CD}_{3} \mathrm{CN}$ as solvent(S). Compounds $\left(\mathbf{1 3}^{1}, \mathbf{1 4}^{2}\right.$, and $\left.\mathbf{1 5}^{3}\right)$ were synthesized according to literature known procedures. 


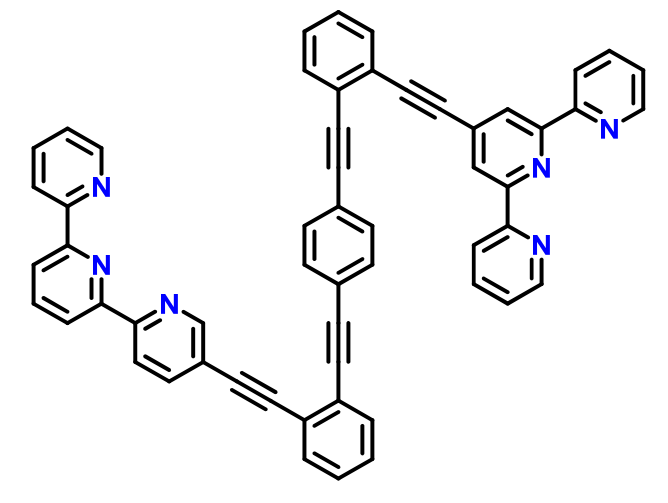

1

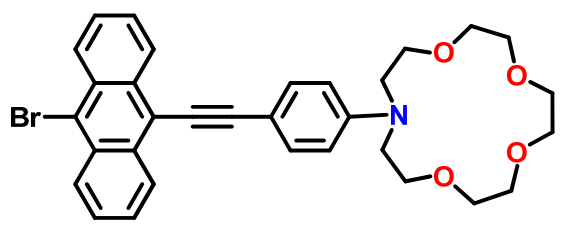

2<smiles>c1ccc(-c2cccc(-c3ccccn3)n2)nc1</smiles>

3

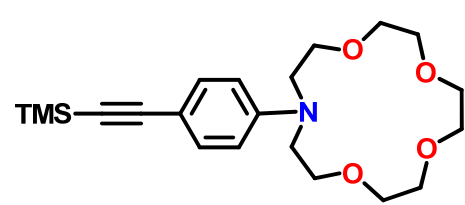

4<smiles>CC(C)(C)C#Cc1ccccc1/C=N/O</smiles>

A<smiles>CC(C)(C)c1cc2ccccc2c[n+]1[O-]</smiles>

B

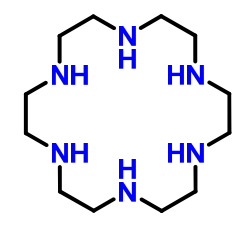

Hexacyclen

Chart 1. Ligands used in the present study.<smiles>OCN(CO)c1ccccc1</smiles>

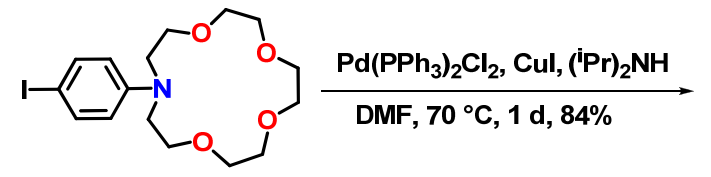

11

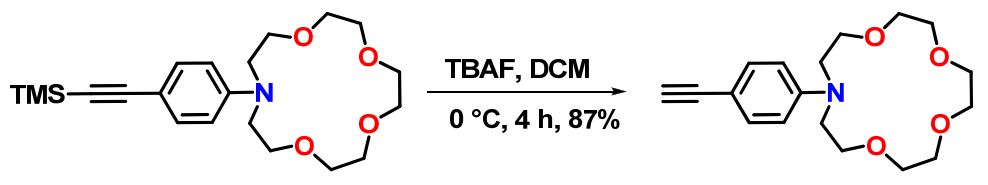

4<smiles>C#Cc1ccc(N2CCOCCOCCOCCOCC2)cc1</smiles>

12

Scheme S1. Synthetic scheme used in the preparation of ligand 2 


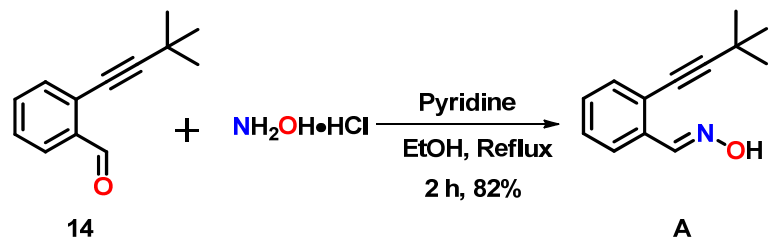<smiles>O=Cc1ccccc1C#Cc1ccccc1</smiles>

15

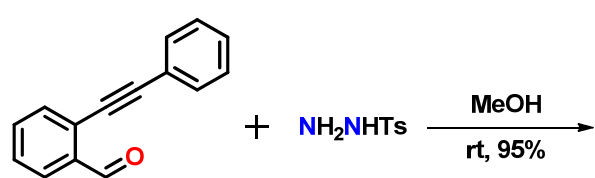

15

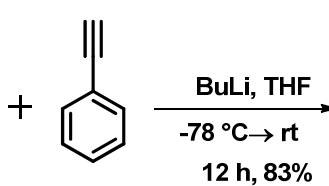<smiles>OC(C#Cc1ccccc1)c1ccccc1C#Cc1ccccc1</smiles><smiles>O=S(=O)(N/N=C/c1ccccc1C#Cc1ccccc1)c1ccccc1</smiles>

E

Scheme S2. Synthetic scheme used in the preparation substrate for catalysis.

\section{Characterization and metal complexes of nanoswitch 1}

\subsection{Characterization of nanoswitch 1}

The preparation of this and related nanoswitches will be presented in a separate manuscript. For inspection all analytical characterization data are provided below.

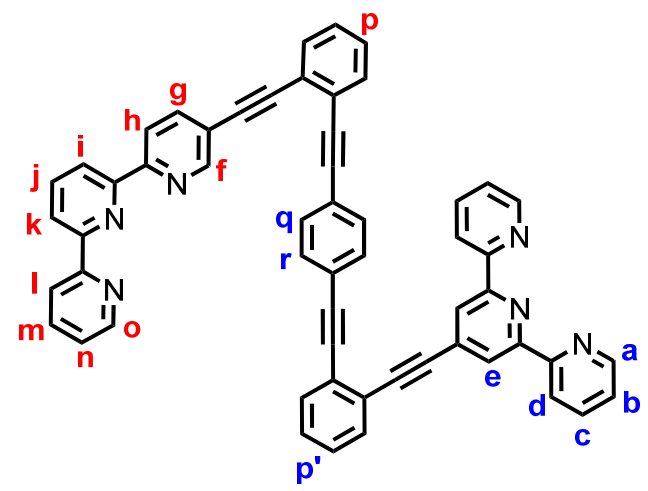

Mp: $220{ }^{\circ} \mathrm{C} .{ }^{1} \mathbf{H}$ NMR (400 MHz, $\left.\mathbf{C D C l}_{3}\right): \delta=7.24-7.28$ (m, 3H, b-, n-H), 7.37-7.41 (m, 4H, p'-H), 7.60-7.66 (m, 6H, p-, q-H), 7.72-7.78 (m, 5H, c-, m-, r-H), 7.87-7.91 (m, 2H, g-, j-H), $8.39\left(\mathrm{dd},{ }^{3} \mathrm{~J}=8.0 \mathrm{~Hz},{ }^{4} \mathrm{~J}=1.2 \mathrm{~Hz}, 2 \mathrm{H}, \mathrm{i}-, \mathrm{k}-\mathrm{H}\right), 8.46-8.51$ (m, 4H, d-, 1-, h-H), 8.63-8.65 (m, 3H, e-, o-H), $8.68\left(\mathrm{ddd},{ }^{3} J=5.2 \mathrm{~Hz},{ }^{4} J=1.6 \mathrm{~Hz},{ }^{5} J=0.8 \mathrm{~Hz}, 2 \mathrm{H}, \mathrm{a}-\mathrm{H}\right), 8.80\left(\mathrm{dd},{ }^{4} J=1.6 \mathrm{~Hz},{ }^{5} J=\right.$ $0.8 \mathrm{~Hz}, 1 \mathrm{H}, \mathrm{f}-\mathrm{H}) \mathrm{ppm} .{ }^{13} \mathbf{C}$ NMR (100 $\left.\mathbf{M H z}, \mathbf{C D C l}_{3}\right): \delta=90.0,90.2,90.7,91.7,92.3,92.4,93.8$, 
93.9, 119.2, 120.1, 120.4, 121.0, 121.1, 121.2, 122.8, 123.1, 123.4, 123.7, 124.0, 124.9, 125.1, 125.3, 125.8, 126.2, 128.3, 128.6, 128.9, 131.7, 131.9, 132.0 (2 C), 132.1, 132.3, 133.1, 136.7, 136.8, 137.8, $139.2149 .0,149.1,151.5,154.5,155.0,155.4,155.5,155.6,155.9$ ppm. ESI-MS: $m / z(\%)=789.2(100)[1+\mathrm{H}]^{+}$. Elemental analysis: Calcd. for $\mathrm{C}_{56} \mathrm{H}_{32} \mathrm{~N}_{6} \bullet 0.2 \mathrm{CH}_{2} \mathrm{Cl}_{2}: \mathrm{C}, 83.76$; H, 4.05; N, 10.43. Found C, 83.77; H, 3.94; N, 10.29 .

\subsection{Synthesis and characterization metal complexes of nanoswitch 1:}

\section{Complex $[\operatorname{Ag}(1)]^{+}$}

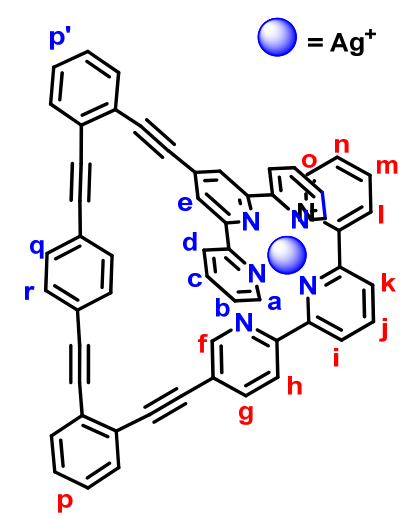

To switch $1(720 \mu \mathrm{g}, 912 \mathrm{nmol})$ placed in an NMR tube, then $\mathrm{AgBF}_{4}(177 \mu \mathrm{g}, 912 \mathrm{nmol})$ in 10 $\mu \mathrm{L}$ of $\mathrm{CD}_{3} \mathrm{CN}$ was added. Afterwards $500 \mu \mathrm{L}$ of $\mathrm{CD}_{2} \mathrm{Cl}_{2}$ was inserted and ${ }^{1} \mathrm{H}$ NMR was recorded. Yield: Quantitative; ${ }^{1} \mathbf{H}$ NMR (400 $\left.\mathbf{~ M H z}, \mathbf{C D}_{\mathbf{2}} \mathbf{C l}_{\mathbf{2}}\right): \delta=7.15\left(\mathrm{ddd},{ }^{3} J=7.6 \mathrm{~Hz},{ }^{3} \mathrm{~J}=\right.$ $\left.4.8 \mathrm{~Hz},{ }^{4} J=1.2 \mathrm{~Hz}, 1 \mathrm{H}, \mathrm{n}-\mathrm{H}\right), 7.20\left(\mathrm{ddd},{ }^{3} J=8.0 \mathrm{~Hz},{ }^{3} J=5.2 \mathrm{~Hz},{ }^{4} J=1.6 \mathrm{~Hz}, 2 \mathrm{H}, \mathrm{b}-\mathrm{H}\right), 7.36-$ 7.43 (m, 4H, q/r-,p'-H), 7.49 (dd, $\left.{ }^{4} J=2.0 \mathrm{~Hz},{ }^{5} J=0.8 \mathrm{~Hz}, 1 \mathrm{H}, \mathrm{f}-\mathrm{H}\right), 7.45-7.48$ (m, 4H, r/q-, p'H), 7.49- 7.52 (m, 1H, h-H), 7.60-7.69 (m, 4H, p-H), 7.70 (ddd, ${ }^{3} J=8.0 \mathrm{~Hz},{ }^{3} J=7.6 \mathrm{~Hz},{ }^{4} J=1.6$ $\mathrm{Hz}, 2 \mathrm{H}, \mathrm{c}-\mathrm{H}), 7.80\left(\mathrm{ddd},{ }^{3} J=8.0 \mathrm{~Hz},{ }^{3} J=7.6 \mathrm{~Hz},{ }^{4} \mathrm{~J}=1.4 \mathrm{~Hz}, 2 \mathrm{H}, \mathrm{m}-\mathrm{H}\right), 7.85\left(\mathrm{dd},{ }^{3} J=5.2 \mathrm{~Hz}\right.$, $\left.{ }^{4} J=1.6 \mathrm{~Hz}, 2 \mathrm{H}, \mathrm{a}-\mathrm{H}\right), 7.98-8.05$ (m, 2H, g-, 1-H), 8.06-8.10 (m, $\left.2 \mathrm{H}, \mathrm{d}-\mathrm{H}\right), 8.13$ (dd, ${ }^{3} J=4.8 \mathrm{~Hz}$, $\left.{ }^{4} J=1.4 \mathrm{~Hz}, 1 \mathrm{H}, \mathrm{o}-\mathrm{H}\right), 8.24-8.28$ (m, 2H, j-,i/k-H), 7.30 (s, 2H, e-H), 8.34 (m, 1H, k/i-H) ppm; ESI-MS: $m / z \quad(\%)=895.8(100) \quad[\operatorname{Ag}(\mathbf{1})]^{+}$. Elemental analysis: Calcd. for $\mathrm{C}_{56} \mathrm{H}_{32} \mathrm{AgBF}_{4} \mathrm{~N}_{6} \bullet 0.5 \mathrm{CH}_{2} \mathrm{Cl}_{2}: \mathrm{C}, 66.14 ; \mathrm{H}, 3.24 ; \mathrm{N}$, 8.19. Found: C, 66.32; H, 3.10; N, 8.37. 


\section{Complex $[\operatorname{Zn}(1)]^{2+}$}

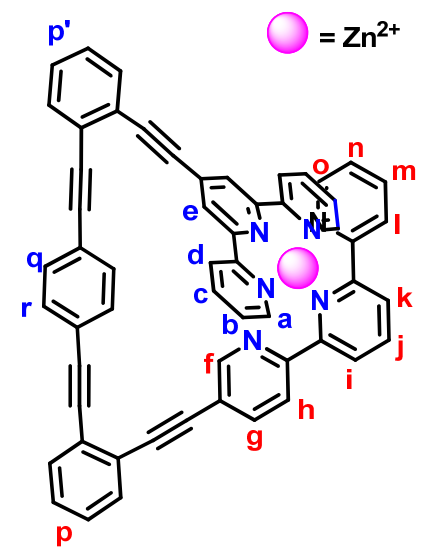

A solution of $\mathrm{Zn}(\mathrm{OTf})_{2}(299 \mu \mathrm{g}, 823 \mathrm{nmol})$ in $\mathrm{CD}_{3} \mathrm{CN}$ was added to switch 1 (650 $\left.\mu \mathrm{g}, 823 \mathrm{nmol}\right)$ in an NMR tube. To this mixture, $500 \mu \mathrm{L}$ of $\mathrm{CD}_{2} \mathrm{Cl}_{2}$ was added and subsequently the NMR was recorded. Yield: Quantitative; ${ }^{1} \mathbf{H}$ NMR (400 MHz, $\left.\mathbf{C D}_{2} \mathbf{C l}_{2}\right): \delta=7.27\left(\mathrm{dd},{ }^{4} J=2.0 \mathrm{~Hz},{ }^{5} \mathrm{~J}=0.8\right.$ Hz, 1H, f-H), 7.29 (d, $\left.{ }^{3} J=8.0 \mathrm{~Hz}, 2 \mathrm{H}, \mathrm{r} / \mathrm{q}-\mathrm{H}\right), 7.37$ (d, $\left.{ }^{3} J=8.0 \mathrm{~Hz}, 2 \mathrm{H}, \mathrm{q} / \mathrm{r}-\mathrm{H}\right), 7.39-7.44$ (m, 3H, n-, p'-H), 7.46- 7.52 (m, 4H, c-, p'-H), 7.54- 7.58 (m, 2H, p-H), 7.65-7.69 (m, 2H, p-H), 7.74 $\left(\mathrm{dd},{ }^{3} J=7.6 \mathrm{~Hz},{ }^{5} J=0.8 \mathrm{~Hz}, 1 \mathrm{H}, \mathrm{h}-\mathrm{H}\right), 7.82\left(\mathrm{ddd},{ }^{3} \mathrm{~J}=5.2 \mathrm{~Hz},{ }^{4} \mathrm{~J}=1.6 \mathrm{~Hz},{ }^{5} \mathrm{~J}=0.8 \mathrm{~Hz}, 2 \mathrm{H}\right.$, aH), 7.05-8.10 (m, 2H, m-, g-H), 8.15 (ddd, $\left.{ }^{3} J=8.0 \mathrm{~Hz},{ }^{3} J=5.2 \mathrm{~Hz},{ }^{4} J=1.6 \mathrm{~Hz}, 2 \mathrm{H}, \mathrm{b}-\mathrm{H}\right), 8.45$ $\left(\mathrm{dd},{ }^{3} J=7.6 \mathrm{~Hz},{ }^{4} J=2.0 \mathrm{~Hz}, 1 \mathrm{H}, 1-\mathrm{H}\right), 8.49\left(\mathrm{td},{ }^{3} J=8.0 \mathrm{~Hz},{ }^{4} J=0.8 \mathrm{~Hz}, 2 \mathrm{H}, \mathrm{d}-\mathrm{H}\right), 8.71\left(\mathrm{dd},{ }^{3} J=\right.$ $\left.7.6 \mathrm{~Hz},{ }^{4} J=1.2 \mathrm{~Hz}, 1 \mathrm{H}, \mathrm{i} / \mathrm{k}-\mathrm{H}\right), 7.78\left(\mathrm{t},{ }^{3} J=7.6 \mathrm{~Hz}, 1 \mathrm{H}, \mathrm{j}-\mathrm{H}\right), 8.83\left(\mathrm{dd},{ }^{3} J=4.8 \mathrm{~Hz},{ }^{4} J=2.0 \mathrm{~Hz}\right.$, 1H, o-H), 8.84 (s, 2H, e-H), $8.98\left(\mathrm{dd},{ }^{3} J=7.6 \mathrm{~Hz},{ }^{4} J=1.2 \mathrm{~Hz}, 1 \mathrm{H}, \mathrm{k} / \mathrm{i}-\mathrm{H}\right)$ ppm; ESI-MS: $m / z$ $(\%)=895.8(100)[\mathrm{Zn}(\mathbf{1})]^{2+} . \mathrm{C}_{58} \mathrm{H}_{32} \mathrm{~F}_{6} \mathrm{~N}_{6} \mathrm{O}_{6} \mathrm{~S}_{2} \mathrm{Zn} \bullet \mathrm{CH}_{2} \mathrm{Cl}_{2}: \mathrm{C}, 57.27 ; \mathrm{H}, 2.77 ; \mathrm{N}, 6.79$. Found: $\mathrm{C}$, $57.12 ; \mathrm{H}, 2.41 ; \mathrm{N}, 6.91$.

\section{Synthesis of ligand 2}

\section{3-Phenyl-1,4,7,10-tetraoxa-13-azacyclopentadecane (10)}

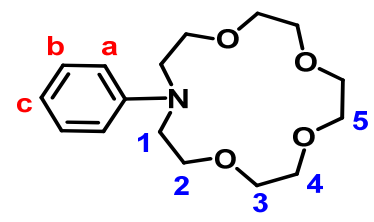

A two-necked flask equipped with a dropping funnel was evacuated and refilled with nitrogen, and then $350 \mathrm{ml}$ dry THF and $\mathrm{NaH}(521 \mathrm{mg}, 13.04 \mathrm{mmol})$ were added and heated up. At reflux temperature, 2,2'-(phenylazanediyl)diethanol (1.00 g, $6.52 \mathrm{mmol})$ and (ethane-1,2-diylbis(oxy))- 
bis(ethane-2,1-diyl) bis(4-methylbenzenesulfonate) $(2.99 \mathrm{~g}, 6.52 \mathrm{mmol})$ were dissolved in 200 $\mathrm{mL}$ of dry THF and then slowly added dropwise to the reaction mixture. Addition was completed in $5 \mathrm{~h}$, and refluxing was continued for another $24 \mathrm{~h}$. After cooling, the solid was filtered and washed with THF. After concentrating the filtrate the brown residue was dissolved in dichloromethane and purified by column chromatography on silica gel (silica gel, EtOAc/DCM $\left.=1: 9, R_{\mathrm{f}}=0.3\right)$ providing $1.15 \mathrm{~g}$ of $\mathbf{1 0}$ as white solid (3.91 mmol, 60\%). Mp: $45^{\circ} \mathrm{C}$. IR (KBr): $\widetilde{v}=979,1005,1038,1059,1091,1122,1132,1176,1192,1226,1243,1269,1292,1323,1344$, 1356, 1379, 1393, 1452, 1470, 1506, 1572, 1599, 2798, 2868, 2889, 2947, 2990, 3024, 3061, $3086 \mathrm{~cm}^{-1}$. ${ }^{1} \mathbf{H}$ NMR (400 MHz, $\left.\mathbf{C D C l}_{3}\right): \delta=3.59\left(\mathrm{t},{ }^{3} J=6.0 \mathrm{~Hz}, 4 \mathrm{H}, 1-\mathrm{H}\right), 3.62-3.70(\mathrm{~m}, 12 \mathrm{H}$, 3-, 4-, 5-H), $3.76\left(\mathrm{t},{ }^{3} \mathrm{~J}=6.0 \mathrm{~Hz}, 4 \mathrm{H}, 2-\mathrm{H}\right), 6.63-6.69$ (m, 3H, a-,c-H), 7.17-7.23 (m, 2H, b-H) ppm; ${ }^{13} \mathbf{C}$ NMR (100 MHz, $\left.\mathbf{C D C l}_{3}\right): \delta=52.33$ 68.47, 70.03, 70.05, 71.19, 111.23, 115.57, 129.15, 147.41 ppm; . ESI-MS: $m / z(\%)=296.4(100)[10+H]^{+}$; Elemental analysis: Calcd. for $\mathrm{C}_{16} \mathrm{H}_{25} \mathrm{NO}_{4} \bullet 0.1 \mathrm{H}_{2} \mathrm{O}: \mathrm{C}, 64.67 ; \mathrm{H}, 8.55 ; \mathrm{N}, 4.71$. Found: C, 64.41; H, 8.64; N, 4.93.

\section{3-(4-Iodophenyl)-1,4,7,10-tetraoxa-13-azacyclopentadecane (11)}

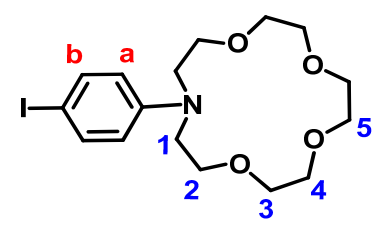

To a solution of compound $\mathbf{1 0}(2.00 \mathrm{~g}, 6.77 \mathrm{mmol})$ in $\mathrm{CH}_{2} \mathrm{Cl}_{2}(30 \mathrm{~mL})$ were added $\mathrm{KI}(1.34 \mathrm{~g}$, $8.13 \mathrm{mmol})$ and $\mathrm{H}_{5} \mathrm{IO}_{6}\left(1.34 \mathrm{~g}, 8.13 \mathrm{mmol}\right.$, dissolved in $30 \mathrm{~mL}$ of $\left.\mathrm{H}_{2} \mathrm{O}\right)$. The mixture was stirred vigorously for $30 \mathrm{~min}$ at r.t. After completion of reaction, which was indicated by TLC analysis, the mixture was washed with a $10 \%$ aq. $\mathrm{Na}_{2} \mathrm{~S}_{2} \mathrm{O}_{3}$ soln. $(30 \mathrm{ml})$. The aqueous fraction was extracted with $\mathrm{CH}_{2} \mathrm{Cl}_{2}(3 \times 20 \mathrm{ml})$. The organic layer was dried $\left(\mathrm{Na}_{2} \mathrm{SO}_{4}\right)$, and the solvent was removed by simple distillation to give a crude product. Further purification was carried out by crystallization from cold hexane to afford the product 11 (2.56 g, $90 \%$ ). Mp: $52{ }^{\circ} \mathrm{C}$. IR (KBr): $\tilde{v}=1007,1041,1052,1095,1125,1132,1176,1192,1226,1243,1269,1292,1323,1344$, 1356, 1379, 1393, 1452, 1470, 1506, 1572, 1599, 2798, 2868, 2889, 2947, 2990, 3024, $3061 \mathrm{~cm}^{-}$ 1. ${ }^{1} \mathbf{H}$ NMR (400 MHz, $\left.\mathbf{C D C l}_{3}\right): \delta=3.53\left(\mathrm{t},{ }^{3} J=6.0 \mathrm{~Hz}, 4 \mathrm{H}, 2-\mathrm{H}\right), 3.61-3.69$ (m, 12H, 3-, 4-, 5H), $3.72\left(\mathrm{t},{ }^{3} J=6.0 \mathrm{~Hz}, 4 \mathrm{H}, 1-\mathrm{H}\right), 6.45\left(\mathrm{~d},{ }^{3} J=8.8 \mathrm{~Hz}, 2 \mathrm{H}, \mathrm{a}-\mathrm{H}\right), 7.43\left(\mathrm{~d},{ }^{3} J=8.8 \mathrm{~Hz}, 2 \mathrm{H}, \mathrm{b}-\mathrm{H}\right)$ 
ppm; ${ }^{13} \mathbf{C}$ NMR (100 MHz, $\left.\mathbf{C D C l}_{3}\right): \delta=52.4,68.2,70.0,70.1,71.3,113.79,137.68$ (2C), 147.10 ppm. . ESI-MS: $m / z(\%)=422.08(100)[11+H]^{+}$. Elemental analysis: Calcd. for $\mathrm{C}_{16} \mathrm{H}_{24} \mathrm{INO}_{4}$ : C, 45.62; H, 5.74; N, 3.32. Found: C, 45.51; H, 5.71; N, 3.20.

\section{3-(4-((Trimethylsilyl)ethynyl)phenyl)-1,4,7,10-tetraoxa-13-azacyclopentadecane (4)}

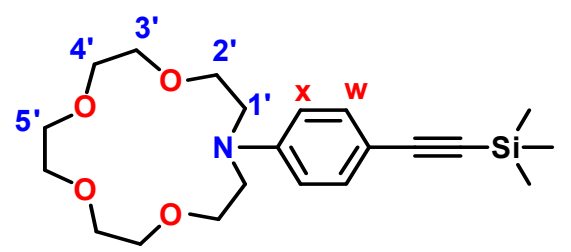

Compound 11 (220 mg, $522 \mu \mathrm{mol}), \mathrm{Pd}\left(\mathrm{PPh}_{3}\right)_{2} \mathrm{Cl}_{2}$ (37.0 mg, $\left.52.2 \mu \mathrm{mol}\right)$ and $\mathrm{CuI}(10 \mathrm{mg}, 52.2$ $\mu \mathrm{mol})$ were loaded in a Schlenk flask under nitrogen atmosphere. Into the mixture, $15 \mathrm{~mL}$ of DMF, $5 \mathrm{~mL}$ of di-isopropylamine and trimethylsilylacetylene $(0.371 \mathrm{~mL}, 2.61 \mathrm{mmol})$ were added and was heated at $70{ }^{\circ} \mathrm{C}$ for $24 \mathrm{~h}$. Thereafter the solvent was removed and the resulting solid was dissolved in DCM and then it was purified by column chromatography $\left(\mathrm{SiO}_{2}\right)$ using DCM as mobile phase $\left(R_{\mathrm{f}}=0.4\right.$ in $50 \% \mathrm{DCM}$ in $\left.\mathrm{SiO}_{2}\right)$ to afford $180 \mathrm{mg}(460 \mu \mathrm{mol}, 88 \%)$ of pure product 4. $\mathbf{M p}: 48{ }^{\circ} \mathrm{C}$. IR (KBr): $\tilde{v}=877,939,985,1124,1181,1248,1294,1354,1387$, 1470, 1516, 1607, 2096, 2873, 2959, 3041, $3278 \mathrm{~cm}^{-1}$. ${ }^{1} \mathbf{H}$ NMR (400 MHz, $\left.\mathbf{C D C l}_{3}\right): \delta=0.22$ (s, 9H, $\left.\mathrm{SiMe}_{3}-\mathrm{H}\right), 3.58\left(\mathrm{t},{ }^{3} J=6.0 \mathrm{~Hz}, 4 \mathrm{H}, 2^{\prime}-\mathrm{H}\right), 3.62-3.69$ (m, 12H, 3'-, 4'-, 5'-H), 3.73 (t, ${ }^{3} J=$ $6.0 \mathrm{~Hz}, 4 \mathrm{H}, 1$ '-H), 6.54 (d, $\left.{ }^{3} \mathrm{~J}=8.8 \mathrm{~Hz}, 2 \mathrm{H}, \mathrm{x}-\mathrm{H}\right), 7.29$ (d, $\left.{ }^{3} J=8.8 \mathrm{~Hz}, 2 \mathrm{H}, \mathrm{w}-\mathrm{H}\right)$ ppm. ${ }^{13} \mathbf{C}$ NMR $\left(\mathbf{1 0 0} \mathbf{~ M H z}, \mathbf{C D C l}_{3}\right): \delta=0.2,52.5,68.3,70.1,70.2,71.3,91.1,106.4,109.5,111.0,133.2,147.5$ ppm. ESI-MS: $m / z(\%)=392.6(100)[4+\mathrm{H}]^{+}$. Elemental analysis: Calcd. for $\mathrm{C}_{21} \mathrm{H}_{33} \mathrm{NO}_{4} \mathrm{Si}: \mathrm{C}$, 64.41; H, 8.41; N, 3.58. Found: C, 64.33; H, 8.15; N, 3.35.

\section{3-(4-Ethynylphenyl)-1,4,7,10-tetraoxa-13-azacyclopentadecane (12)}

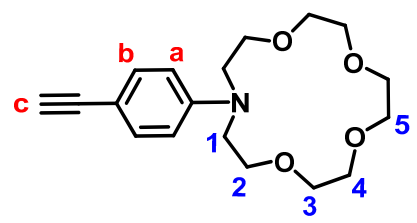


Compound 4 (170 mg, $434 \mu \mathrm{mol})$ was dissolved in $50 \mathrm{~mL}$ of DCM. Then TBAF (397 mg, 1.51 mmol) in DCM $(10 \mathrm{~mL})$ was added at $0{ }^{\circ} \mathrm{C}$ and the reaction mixture was stirred for $4 \mathrm{~h}$ at the same temperature. Then the reaction mixture was poured into a separating funnel containing 50 $\mathrm{mL}$ of DCM and $30 \mathrm{~mL}$ of $\mathrm{H}_{2} \mathrm{O}$. The organic layer was separated, dried with $\mathrm{Na}_{2} \mathrm{SO}_{4}$, and filtered. The solution was concentrated under vacuum to furnish a white solid that was purified by column chromatography (silica gel, EtOAc/hexane $=1: 9, \mathrm{R}_{f}=0.4$ ) to afford $124 \mathrm{mg}$ (317 $\mu \mathrm{mol}, \mathbf{9 0 \%}$ ) of the product 12 as white solid; mp: $40{ }^{\circ} \mathrm{C}$. IR (KBr): $\tilde{v}=817,875,941,982$, 1120, 1175, 1249, 1290, 1348, 1395, 1468, 1512, 1601, 2870, 2951, 3045, $3270 \mathrm{~cm}^{-1}$. ${ }^{\mathbf{1}} \mathbf{H}$ NMR (400 MHz, $\left.\mathbf{C D C l}_{3}\right): \delta=2.95(\mathrm{~s}, 1 \mathrm{H}, \mathrm{c}-\mathrm{H}), 3.58\left(\mathrm{t},{ }^{3} J=6.0 \mathrm{~Hz}, 4 \mathrm{H}, 2-\mathrm{H}\right), 3.62-3.69(\mathrm{~m}, 12 \mathrm{H}, 3-$, 4-, 5-H), $3.73\left(\mathrm{t},{ }^{3} \mathrm{~J}=6.0 \mathrm{~Hz}, 4 \mathrm{H}, 1-\mathrm{H}\right), 6.55\left(\mathrm{~d},{ }^{3} \mathrm{~J}=8.8 \mathrm{~Hz}, 2 \mathrm{H}, \mathrm{a}-\mathrm{H}\right), 8.8\left(\mathrm{~d},{ }^{3} J=7.43 \mathrm{~Hz}, 2 \mathrm{H}\right.$, b-H) ppm. ${ }^{13} \mathbf{C}$ NMR (100 MHz, $\left.\mathbf{C D C l}_{3}\right): \delta=52.4,68.2,70.0,70.1,71.2,74.7,84.7,108.3$, 111.0, 133.3, 147.7 ppm. ESI-MS: $m / z(\%)=320.5(100)[12+H]^{+}$; Elemental analysis: Calcd. for $\mathrm{C}_{18} \mathrm{H}_{25} \mathrm{NO}_{4} \bullet 0.5 \mathrm{H}_{2} \mathrm{O}: \mathrm{C}, 5.83 ; \mathrm{H}, 7.98 ; \mathrm{N}, 4.27$. Found: C, 65.87; H, 7.68; N, 4.53.

\section{3-(4-((10-Bromoanthracen-9-yl)ethynyl)phenyl)-1,4,7,10-tetraoxa-13-azacyclopentadecane}

(2)

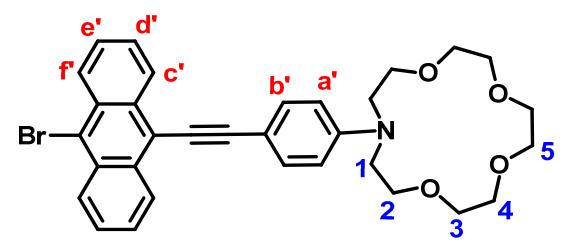

Under argon atmosphere 9-bromo-10-iodoanthracene (13) (155 mg, $407 \mu \mathrm{mol})$ and compound $12(100 \mathrm{mg}, 313 \mu \mathrm{mol})$ were dissolved in $15 \mathrm{~mL}$ of dry DMF and $15 \mathrm{~mL}$ of di-isopropylamine. Subsequently the solution was deaerated by bubbling nitrogen through the solution for $1 \mathrm{~h}$. Then the solution was charged with $\mathrm{Pd}\left(\mathrm{PPh}_{3}\right)_{4}(36.0 \mathrm{mg}, 31.3 \mu \mathrm{mol})$ and heated at $70{ }^{\circ} \mathrm{C}$ for $24 \mathrm{~h}$. The resulting solution was evaporated to dryness and the crude product was purified by column chromatography (silica gel, EtOAc/hexane $=4: 6, \mathrm{R}_{f}=0.5$ ) providing $125 \mathrm{mg}$ of 2 as reddish solid $(217 \mu \mathrm{mol}, 70 \%)$; mp: $82{ }^{\circ} \mathrm{C}$. IR (KBr): $\tilde{v}=536,576,602,622,753,815,876,903,938$, 992, 1119, 1191, 1255, 1285, 1347, 1390, 1436, 1473, 1518, 1605, 2191, 2891, 2858, $3077 \mathrm{~cm}^{-1}$. ${ }^{1} \mathbf{H}$ NMR (400 MHz, $\left.\mathbf{C D}_{2} \mathbf{C l}_{2}\right): \delta=3.59(\mathrm{~s}, 4 \mathrm{H}, 5-\mathrm{H}), 3.63\left(\mathrm{t},{ }^{3} \mathrm{~J}=6.0 \mathrm{~Hz}, 4 \mathrm{H}, 1-\mathrm{H}\right), 3.64(\mathrm{~s}, 8 \mathrm{H}$, 3-, 4-H), 3.77 (t, $\left.{ }^{3} J=6.0 \mathrm{~Hz}, 4 \mathrm{H}, 2-\mathrm{H}\right), 6.75$ (d, ${ }^{3} J=8.8 \mathrm{~Hz}, 2 \mathrm{H}, \mathrm{a}$ '-H), 7.60-7.69 (m, 6H, b'-, d', e'-H), 8.52-8.58 (m, 2H, c'/f'-H), 8.70-8.75 (m, 2H, f'/c'-H) ppm. ${ }^{13}$ C NMR (100 MHz, 
$\left.\mathbf{C D}_{\mathbf{2}} \mathbf{C l}_{2}\right): \delta=53.0,68.7,70.2,70.6,71.4,84.4,104.4,109.6,148.6,111.9,119.9,122.8,126.9$, $127.8,127.9,128.3,130.7,132.9,133.3 \mathrm{ppm}$. ESI-MS: $m / z(\%)=576.2(100)[2+\mathrm{H}]^{+}$; Elemental analysis: Calcd. for $\mathrm{C}_{32} \mathrm{H}_{32} \mathrm{BrNO}_{4}$ : C, 66.90; H, 5.61; N, 2.44. Found: C, 66.88; H, $5.58 ; \mathrm{N}, 2.36$.

\subsection{Synthesis and characterization of metal complexes of ligand 2}

\section{Complex $[\operatorname{Ag}(2)]^{+}$}

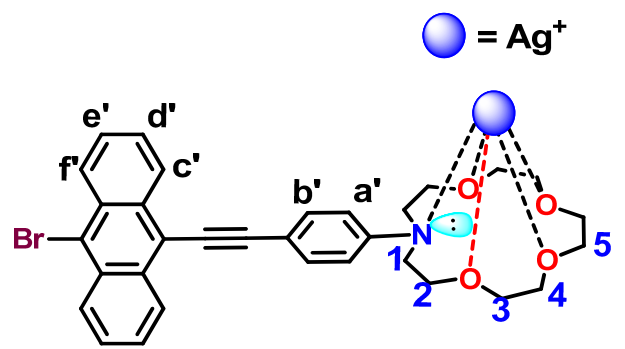

Ligand $2(500 \mu \mathrm{g}, 0.870 \mu \mathrm{mol})$ was placed into an $\mathrm{NMR}$ tube and $\mathrm{AgBF}_{4}(169 \mu \mathrm{g}, 0.870 \mu \mathrm{mol})$ in $10 \mu \mathrm{L}$ of $\mathrm{CD}_{3} \mathrm{CN}$ was added. After insertion of $500 \mu \mathrm{L}$ of $\mathrm{CD}_{2} \mathrm{Cl}_{2}$ the NMR was recorded. Yield: Quantitative. ${ }^{1} \mathbf{H}$ NMR (400 MHz, $\left.\mathbf{C D}_{2} \mathbf{C l}_{2}\right): \delta=3.54\left(\mathrm{t},{ }^{3} \mathrm{~J}=4.0 \mathrm{~Hz}, 4 \mathrm{H}, 1-\mathrm{H}\right), 3.66$ (s, 4H, 5-H), 3.68 (s, 8H, 3-, 4-H), 3.72 (t, $\left.{ }^{3} J=4.0 \mathrm{~Hz}, 4 \mathrm{H}, 2-\mathrm{H}\right), 7.05$ (d, $\left.{ }^{3} J=7.2 \mathrm{~Hz}, 2 \mathrm{H}, \mathrm{a}-\mathrm{H}\right)$, 7.63-7.69 (m, 4H, d'-, e'-H), 7.71 (d, $\left.{ }^{3} J=7.2 \mathrm{~Hz}, 2 \mathrm{H}, \mathrm{b}^{\prime}-\mathrm{H}\right), 8.55-8.60$ (m, 2H, f'/c'-H), 8.69$8.73\left(\mathrm{~m}, 2 \mathrm{H}, \mathrm{c}^{\prime} / \mathrm{f}^{\prime}-\mathrm{H}\right)$. ESI-MS: $m / z(\%)=682.2(100)[\operatorname{Ag}(2)]^{+}$. Elemental analysis: Calcd. for $\mathrm{C}_{32} \mathrm{H}_{32} \mathrm{AgBBrF}_{4} \mathrm{NO}_{4}$ : C, 49.97; H, 4.19; N, 1.82. Found: C, 50.18; H, 4.51; N, 1.91 .

\section{Synthesis and characterization of substrate for the catalysis}

(E)-2-(3,3-Dimethylbut-1-yn-1-yl)benzaldehyde oxime (A) ${ }^{4}$

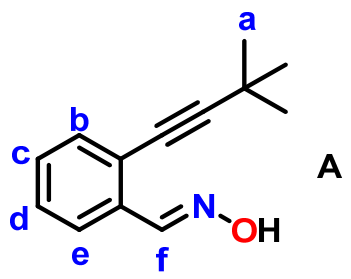

A solution of 2-alkynylbenzaldehyde (14) $(150 \mathrm{mg}, 805 \mu \mathrm{mol})$, hydroxylamine hydrochloride (111 mg $1.61 \mathrm{mmol})$, and pyridine $(129 \mu \mathrm{L}, 1.61 \mathrm{mmol})$ in $\mathrm{C}_{2} \mathrm{H}_{5} \mathrm{OH}(10 \mathrm{~mL})$ was stirred under 
reflux for $2 \mathrm{~h}$. After completion of the reaction as indicated by thin-layer chromatography (TLC), the solvent was evaporated and extracted with AcOEt $(2 \times 30 \mathrm{~mL})$, followed by drying over anhydrous $\mathrm{Na}_{2} \mathrm{SO}_{4}$. After evaporation of the solvent, purification by chromatography (silica gel, $\left.\mathrm{EtOAc} / \mathrm{DCM}=1: 4, \mathrm{R}_{f}=0.5\right)$ provided $132 \mathrm{mg}$ of pure $\mathbf{A}(660 \mu \mathrm{mol}, 82 \%)$. IR $(\mathbf{K B r}): \tilde{v}=491$, $525,559,574,634,665,719,761,791,873,918,935,947,968,1013,1187,1210,1281,1306$, 1360, 1387, 1449, 1471, 1488, 1594, 1932, 2233, 2866, 2927, 2966, 3002, $3256 \mathrm{~cm}^{-1}$. ${ }^{1} \mathbf{H}$ NMR (400 MHz, $\left.\mathbf{C D}_{2} \mathbf{C l}_{2}\right): \delta=1.25(\mathrm{~s}, 9 \mathrm{H}, \mathrm{a}-\mathrm{H}), 7.15-7.25$ (m, 2H, c-, d-H), 7.31-7.35 (m, 1H, b-H), 7.72-7.76 (m, $1 \mathrm{H}, \mathrm{e}-\mathrm{H}), 8.55(\mathrm{~s}, 1 \mathrm{H}, \mathrm{f}-\mathrm{H}) \mathrm{ppm}$. Elemental analysis: Calcd. for $\mathrm{C}_{13} \mathrm{H}_{15} \mathrm{NO}$ : $\mathrm{C}$, 77.58; H, 7.51; N, 6.96. Found: C, 77.41; H, 7.62.; N, 6.81.

\section{3-Phenyl-1-(2-(phenylethynyl)phenyl)prop-2-yn-1-ol (C) ${ }^{5}$}

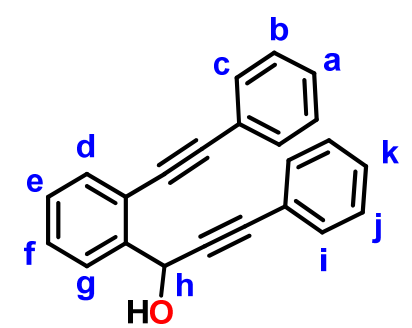

To a solution of ethynylbenzene (16) $(990 \mathrm{mg}, 9.68 \mathrm{mmol})$ in THF $(50 \mathrm{~mL})$ was added $n$-BuLi (2.5 $\mathrm{M}$ in hexane, $3.82 \mathrm{~mL}, 9.68 \mathrm{mmol})$ at $-78{ }^{\circ} \mathrm{C}$. After stirring for $1 \mathrm{~h}$ at this temperature, compound 15 (1.00 g, $4.84 \mathrm{mmol})$ was added and after warming up the resulting mixture was stirred at room temperature for $2 \mathrm{~h}$. After the starting material had been consumed, the mixture was quenched with water, extracted with ethyl acetate, washed with brine and dried over anhydrous $\mathrm{Na}_{2} \mathrm{SO}_{4}$. The solvent was evaporated and the residue was purified by column chromatography (silica gel, EtOAc/Hexane = 1:5) to afford $1.34 \mathrm{~g}(4.35 \mathrm{mmol}, 90 \%)$ of the desired product $\mathbf{C}$ as yellow oil. IR (KBr): $\tilde{v}=525,544,555,584,634,690,755,811,852$, 915, 963, 997, 1031, 1069, 1096, 1159, 1185, 1278, 1310, 1383, 1442, 1491, 1570, 1599, 1623, 1697, 1954, 2216, 3058, $3200 \mathrm{~cm}^{-1} .{ }^{1} \mathbf{H}$ NMR (400 MHz, $\left.\mathbf{C D C l}_{3}\right): \delta=7.26-7.38$ (m, 5H, a-, b-, k-, j-, d-H), 7.41 (td, $\left.{ }^{3} J=7.6 \mathrm{~Hz},{ }^{4} J=1.6 \mathrm{~Hz}, 1 \mathrm{H}, \mathrm{e}-\mathrm{H}\right), 7.44-7.47$ (m, 2H, c-H), 7.54-7.61 (m, $3 \mathrm{H}, \mathrm{f}-, \mathrm{i}-\mathrm{H}), 7.79\left(\mathrm{dd},{ }^{3} J=7.6 \mathrm{~Hz},{ }^{4} J=1.6 \mathrm{~Hz}, 1 \mathrm{H}, \mathrm{g}-\mathrm{H}\right) \mathrm{ppm}$. 
(E)-N'-(2-(Phenylethynyl)benzylidene)benzenesulfonohydrazide (E) ${ }^{6}$

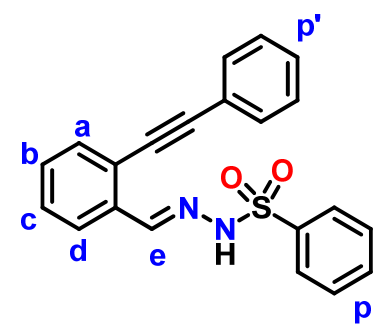

Compound 15 (100 mg, $484 \mu \mathrm{mol})$ and benzenesulfonyl hydrazide $(83.0 \mathrm{mg}, 484 \mu \mathrm{mol})$ in $5 \mathrm{~mL}$ of EtOH were stirred for $12 \mathrm{~h}$. After completion of the reaction the solid precipitate was washed with cold EtOH and dried over vacuum. Product E (141 mg, $387 \mu \mathrm{mol}, 80 \%)$ was isolated as white solid. IR (KBr): $\tilde{v}=531,570,601,622,753,1007,1041,1052,1095,1125,1132,1176$, 1192, 1226, 1243, 1269, 1292, 1323, 1344, 1356, 1379, 1393, 1452, 1470, 1506, 1572, 1599, 1930, 2201, 2798, 2868, 2889, 2947, 2990, 3024, 3061 $\mathrm{cm}^{-1} .{ }^{\mathbf{1}} \mathbf{H}$ NMR (400 MHz, $\left.\mathbf{C D C l}_{3}\right): \delta=$ 7.30-7.40 (m, 5H, p'/p-H), 7.48-7.52 (m, 5H, p/p'-H), 7.57-7.62 (m, 1H, a-H), 7.86 (s, 1H, N-H), 7.92-7.97 (m, 1H, d-H), 7.99-8.03 (m, 2H, b-, c-H), 8.35 (s, 1H, e-H) ppm.

\section{Synthesis and characterization of model complexes}

All solid compounds were placed in an NMR tube and then dissolved in $\mathrm{CD}_{2} \mathrm{Cl}_{2}$. Subsequently, NMR spectra were measured at $298 \mathrm{~K}$. $\mathrm{Zn}(\mathrm{OTf})_{2}$ and $\mathrm{AgBF}_{4}$ were added as a standard solution in $\mathrm{CD}_{3} \mathrm{CN}$.

Model complex $5=\left[\operatorname{Ag}(3)_{2}\right]^{+}$

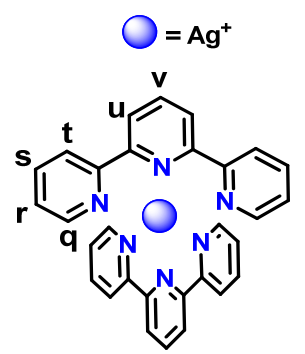

In an NMR tube, 2,2':6',2"-terpyridine (3) $(723 \mu \mathrm{g}, 3.09 \mu \mathrm{mol})$ and $\mathrm{AgBF}_{4}(301 \mu \mathrm{g}, 1.55 \mu \mathrm{mol})$ were dissolved in $500 \mu \mathrm{L}$ of $\mathrm{CD}_{2} \mathrm{Cl}_{2}$ and $\mathrm{CD}_{3} \mathrm{CN}$ (50:1). Yield by NMR: quantitative; IR (KBr): 
$\tilde{v}=521,533,622,631,656,733,765,770,832,895,989,1039,1083,1104,1123,1264,1423$, 1454, 1469, 15621581, 3012, $3050 \mathrm{~cm}^{-1}$. ${ }^{\mathbf{1}} \mathbf{H}$ NMR (400 MHz, $\left.\mathbf{C D C l}_{3}\right): \delta=7.27\left(\mathrm{ddd},{ }^{3} J=7.6\right.$ $\left.\mathrm{Hz},{ }^{3} J=4.8 \mathrm{~Hz},{ }^{4} J=1.2 \mathrm{~Hz}, 4 \mathrm{H}, \mathrm{r}-\mathrm{H}\right), 7.80\left(\mathrm{ddd},{ }^{3} J=8.0 \mathrm{~Hz},{ }^{3} J=7.6 \mathrm{~Hz},{ }^{4} J=2.0 \mathrm{~Hz}, 4 \mathrm{H}, \mathrm{s}-\mathrm{H}\right)$, 8.13-8.17 (m, 5H, t-,v-H), 8.19-8.22 (m, 8H, q-,u-H), ppm. ESI-MS: $m / z(\%)=573.1(100)$ $\left[\left[\operatorname{Ag}(3)_{2}^{+}\right]\right.$.

Model complex $6=\left[\operatorname{Zn}(3)_{2}\right]^{2+}$

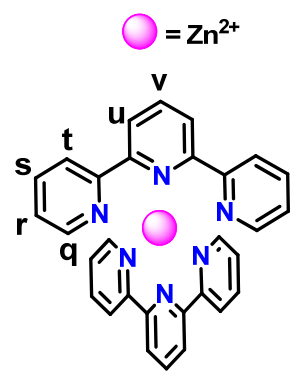

In an NMR tube, 2,2':6',2"-terpyridine (3) (709 $\mu \mathrm{g}, 3.03 \mu \mathrm{mol})$, and Zn(OTf) $2(550 \mu \mathrm{g}, 1.51$ $\mu \mathrm{mol})$ were dissolved in $500 \mu \mathrm{L}$ of $\mathrm{CD}_{2} \mathrm{Cl}_{2}$ and $\mathrm{CD}_{3} \mathrm{CN}$ (50:1). Yield by NMR: quantitative; IR (KBr): $\tilde{v}=517,571,636,651,740,752,766,787,1017,1031,1053,1095,1129,1141,1154$, $1188,1225,1273,1325,1384,1423,1443,1456,1477,1497,1565,1587,1599,3080,3110 \mathrm{~cm}^{-}$ ${ }^{1}{ }^{1} \mathbf{H}$ NMR (400 MHz, $\left.\mathbf{C D C l}_{3}\right): \delta=7.35\left(\mathrm{ddd},{ }^{3} J=7.6 \mathrm{~Hz},{ }^{3} \mathrm{~J}=5.2 \mathrm{~Hz},{ }^{4} \mathrm{~J}=1.2 \mathrm{~Hz}, 4 \mathrm{H}, \mathrm{r}-\mathrm{H}\right)$, $7.65\left(\mathrm{ddd},{ }^{3} J=5.2 \mathrm{~Hz},{ }^{4} J=1.6 \mathrm{~Hz},{ }^{5} J=0.8 \mathrm{~Hz}, 4 \mathrm{H}, \mathrm{q}-\mathrm{H}\right), 8.13-8.17\left(\mathrm{ddd},{ }^{3} J=8.0 \mathrm{~Hz},{ }^{3} J=7.6\right.$ $\left.\mathrm{Hz},{ }^{5} J=1.6 \mathrm{~Hz}, 4 \mathrm{H}, \mathrm{s}-\mathrm{H}\right), 8.51\left(\mathrm{td},{ }^{3} J=8.0 \mathrm{~Hz},{ }^{4} J=0.8 \mathrm{~Hz}, 4 \mathrm{H}, \mathrm{t}-\mathrm{H}\right), 8.65-8.74(\mathrm{~m}, 6 \mathrm{H}, \mathrm{u}-, \mathrm{v}-\mathrm{H})$ ppm. ESI-MS: $m / z(\%)=295.9(15)[6], 679.1(100)\left[6+\right.$ OTf $\left.^{-}\right]$.

\section{Model complex $7=[\operatorname{Ag}(4)]^{+}$}

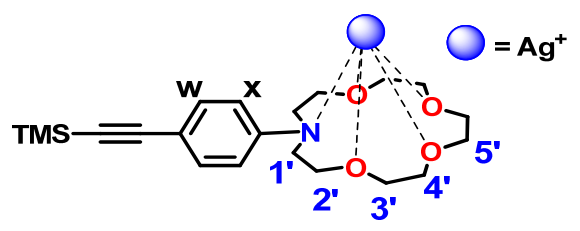

In an NMR tube, compound $4(600 \mu \mathrm{g}, 1.53 \mu \mathrm{mol})$ and $\mathrm{AgBF}_{4}(298 \mu \mathrm{g}, 1.53 \mu \mathrm{mol})$ were dissolved in $500 \mu \mathrm{L}$ of $\mathrm{CD}_{2} \mathrm{Cl}_{2}$ and $\mathrm{CD}_{3} \mathrm{CN}$ (50:1). Yield by NMR: quantitative; IR (KBr): $\widetilde{v}=$ 979, 1005, 1038, 1059, 1091, 1122, 1132, 1176, 1192, 1226, 1243, 1269, 1292, 1323, 1344, 1356, 1379, 1393, 1452, 1470, 1506, 1572, 1599, 2798, 2868, 2889, $2947 \mathrm{~cm}^{-1}$. ${ }^{\mathbf{1}}$ H NMR (400 MHz, $\left.\mathbf{C D C l}_{3}\right): \delta=0.21\left(\mathrm{~s}, 9 \mathrm{H}, \mathrm{SiMe}_{3}-\mathrm{H}\right), 3.43\left(\mathrm{t},{ }^{3} J=4.4 \mathrm{~Hz}, 4 \mathrm{H}, 1\right.$ '-H ), $3.62\left(\mathrm{t},{ }^{3} J=4.4 \mathrm{~Hz}\right.$, 
4H, 2'-H), 3.64 (s, 4H, 5'-H), 3.66 (s, 8H, 3'-, $\left.4^{\prime}-\mathrm{H}\right), 6.95$ (d, $\left.{ }^{3} J=7.2 \mathrm{~Hz}, 2 \mathrm{H}, \mathrm{x}-\mathrm{H}\right), 7.36$ (d, ${ }^{3} J=$ $7.2 \mathrm{~Hz}, 2 \mathrm{H}, \mathrm{w}-\mathrm{H})$. ESI-MS: $m / z(\%)=498.2(100)$ [7].

\section{Model studies}

Metal-dependent self-sorting was tested by mixing with ligand $3(370 \mu \mathrm{g}, 1.58 \mu \mathrm{mol})$, ligand 4 $(622 \mu \mathrm{g}, 1.58 \mu \mathrm{mol}), \mathrm{AgBF}_{4}(307 \mu \mathrm{g}, 1.58 \mu \mathrm{mol})$ and $\mathrm{Zn}(\mathrm{OTf})_{2}(287 \mu \mathrm{g}, 0.790 \mu \mathrm{mol})$ in a ratio of 2:1:1:1 in $500 \mu \mathrm{L}$ of $\mathrm{CD}_{2} \mathrm{Cl}_{2}$ and $\mathrm{CD}_{3} \mathrm{CN}$ (50:1). The ${ }^{1} \mathrm{H}$ NMR spectrum was compared with those of the individual ligands and complexes. Accordingly, $[\operatorname{Ag}(4)]^{+}$and $\left[\operatorname{Zn}(3)_{2}\right]^{2+}$ were formed quantitatively.
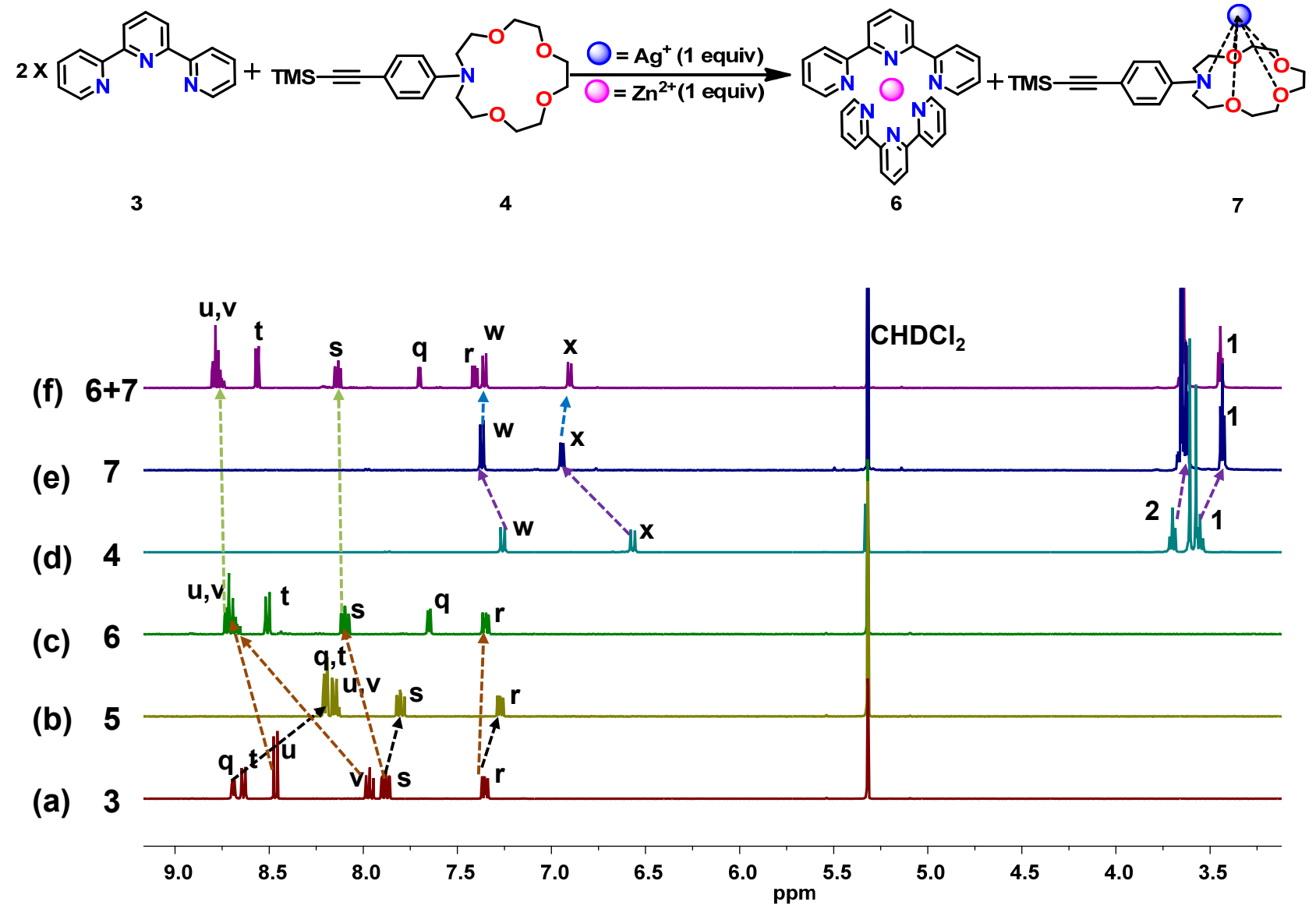

Figure S1. ${ }^{1} \mathrm{H}$ NMR (400 MHz, $298 \mathrm{~K}$ ) of (a) 3; (b) complex $\mathbf{5}=\left[\mathrm{Ag}(\mathbf{3})_{2}\right]^{+}$; (c) complex $\mathbf{6}=\left[\mathrm{Zn}(\mathbf{3})_{2}\right]^{2+}$; (d) $\mathbf{4}$; (e) complex $\mathbf{7}=[\mathrm{Ag}(\mathbf{4})]^{+}$; (f) after mixing of $\mathbf{3}, \mathbf{4}, \mathrm{AgBF}_{4}, \mathrm{Zn}(\mathrm{OTf})_{2}$ in a ratio of 2:1:1:1. 
7. NMR spectra: ${ }^{1} \mathrm{H}$ NMR, ${ }^{13} \mathrm{C}$ NMR, ${ }^{1} \mathrm{H}-{ }^{1} \mathrm{H}$ COSY

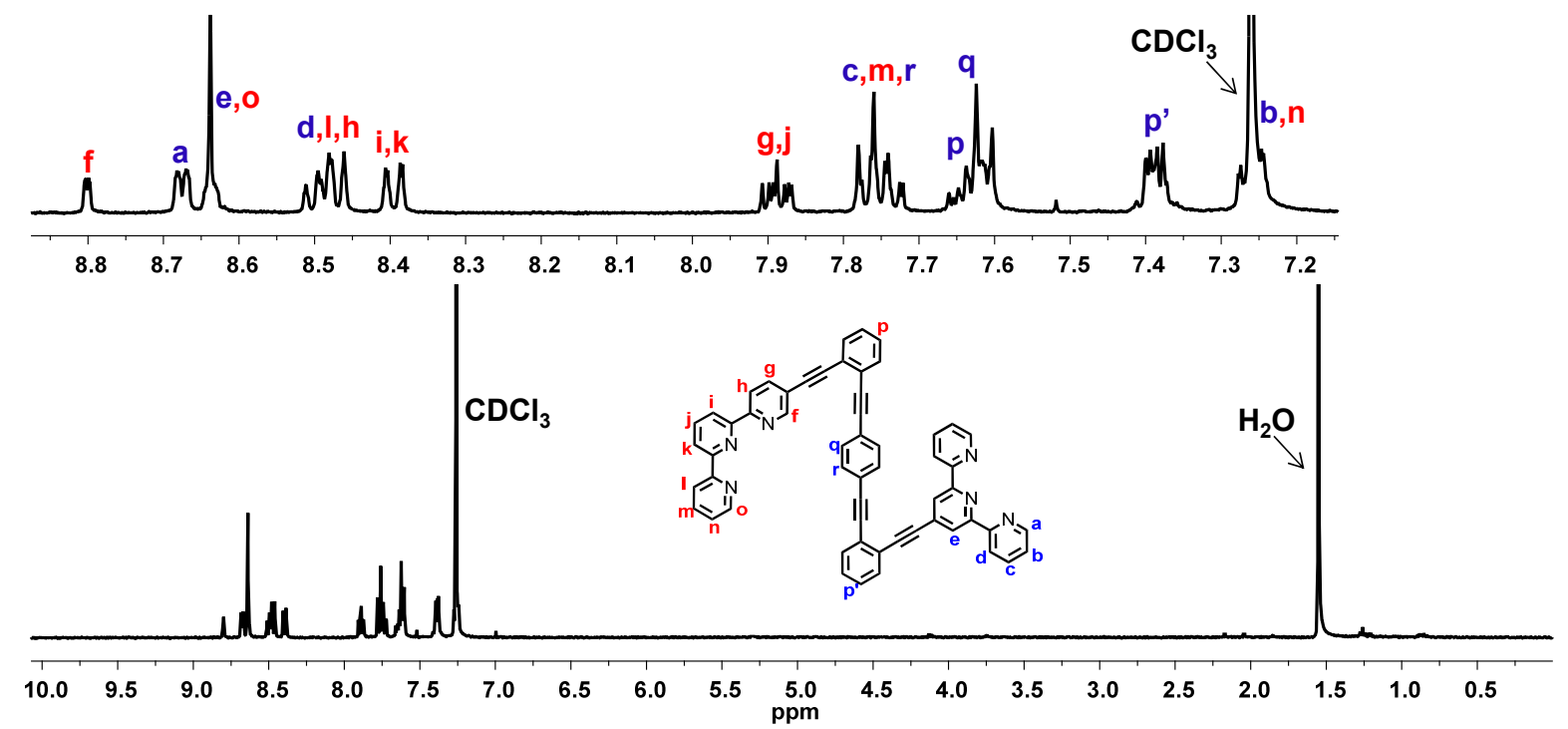

Figure S2. ${ }^{1} \mathrm{H}$ NMR spectrum of 1 in $\mathrm{CDCl}_{3}(400 \mathrm{MHz}, 298 \mathrm{~K})$.

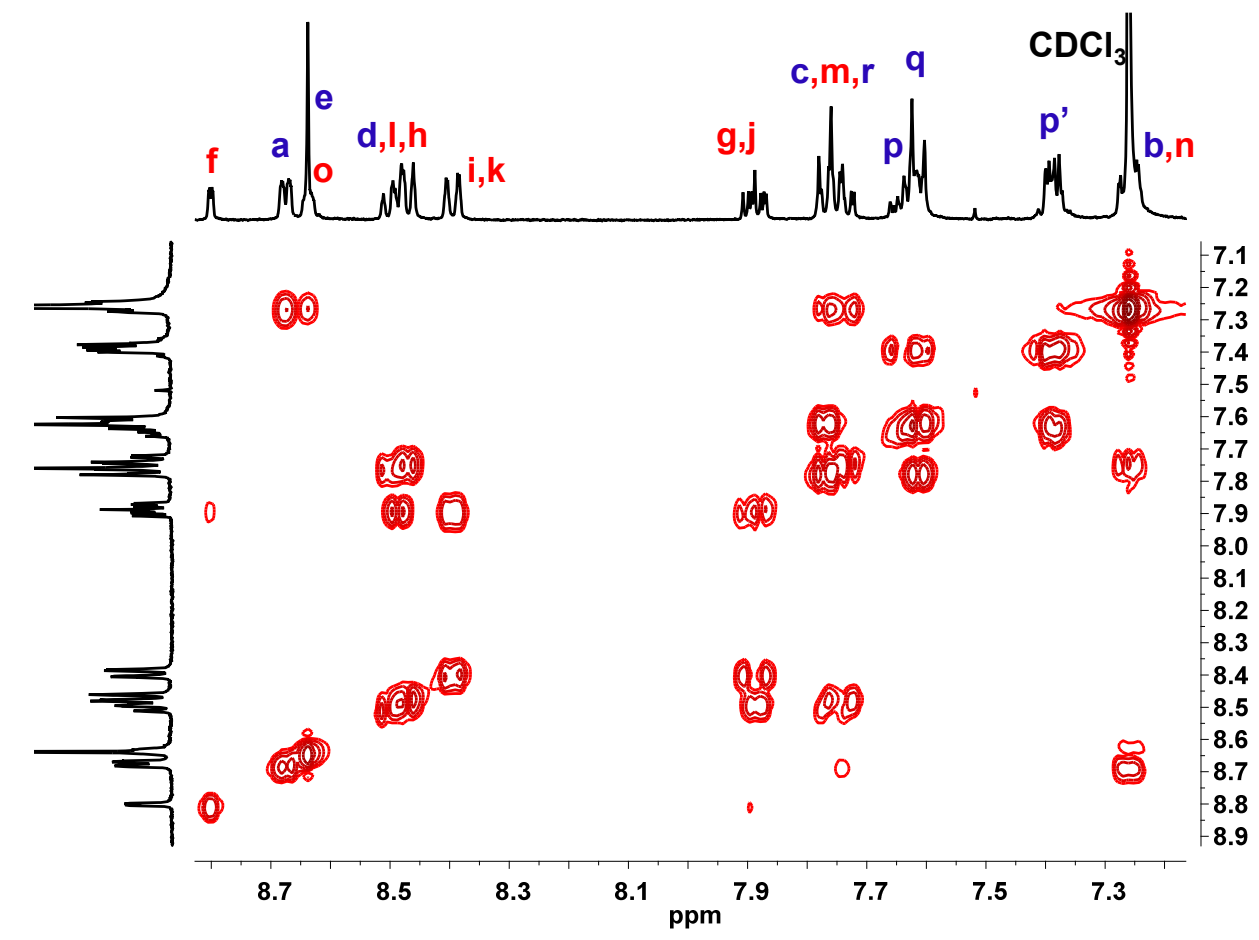

Figure S3. ${ }^{1} \mathrm{H}-{ }^{1} \mathrm{H}$ COSY spectrum of 1 in $\mathrm{CDCl}_{3}(400 \mathrm{MHz}, 298 \mathrm{~K})$. 


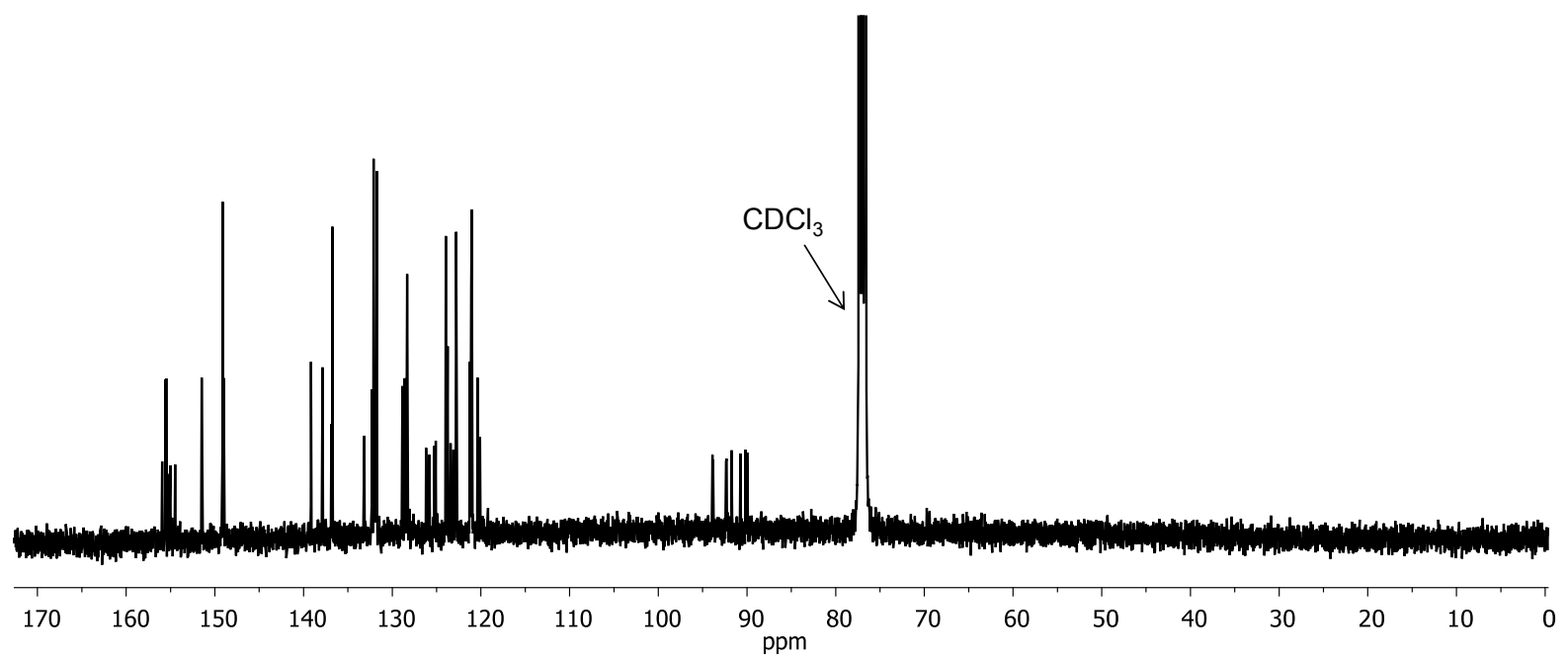

Figure S4. ${ }^{13} \mathrm{C}$ NMR spectrum of $\mathbf{1}$ in $\mathrm{CDCl}_{3}(100 \mathrm{MHz}, 298 \mathrm{~K})$.
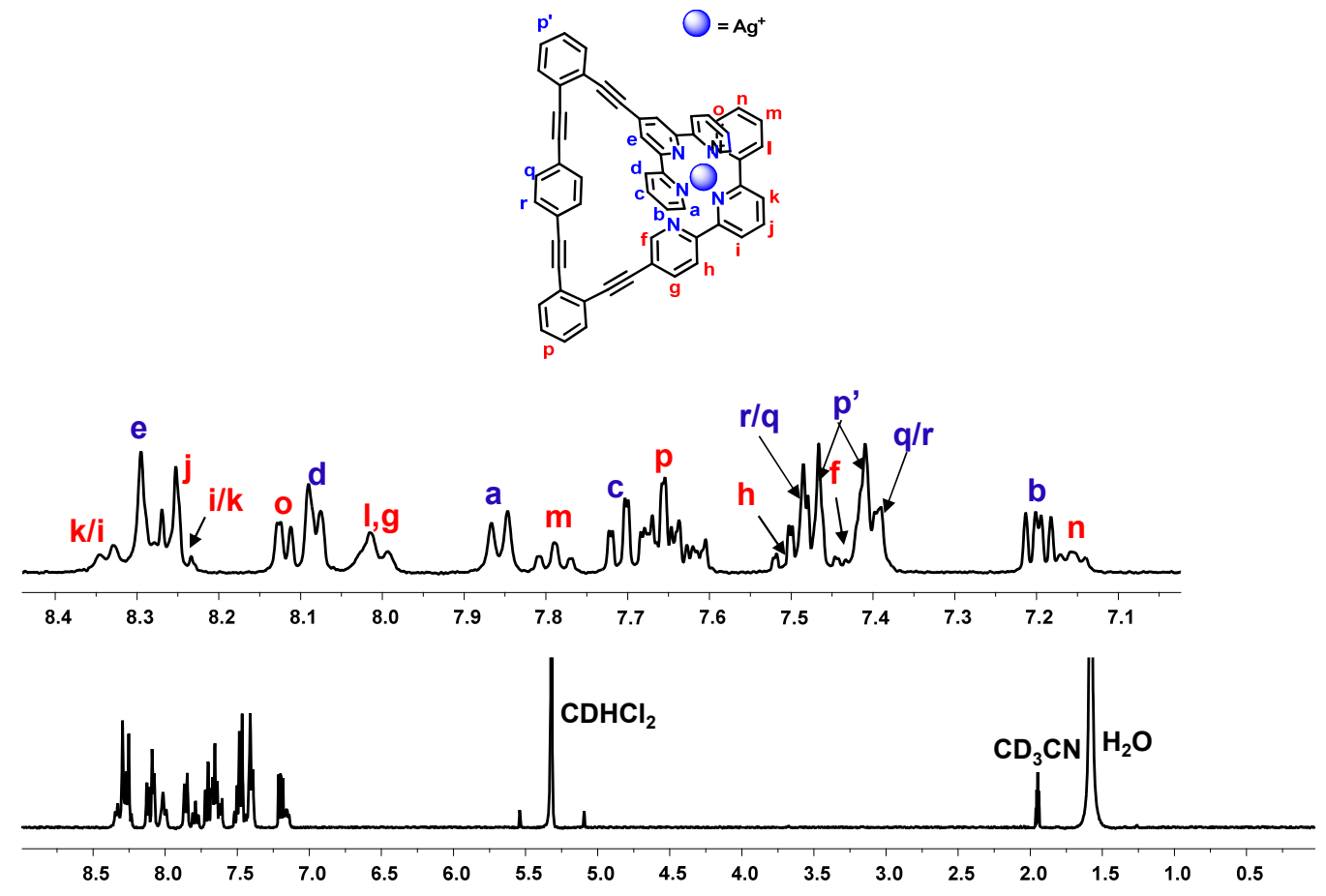

Figure S5. ${ }^{1} \mathrm{H}$ NMR spectrum of complex $[\mathrm{Ag}(\mathbf{1})]^{+}$in $\mathrm{CD}_{2} \mathrm{Cl}_{2}(400 \mathrm{MHz}, 298 \mathrm{~K})$. 


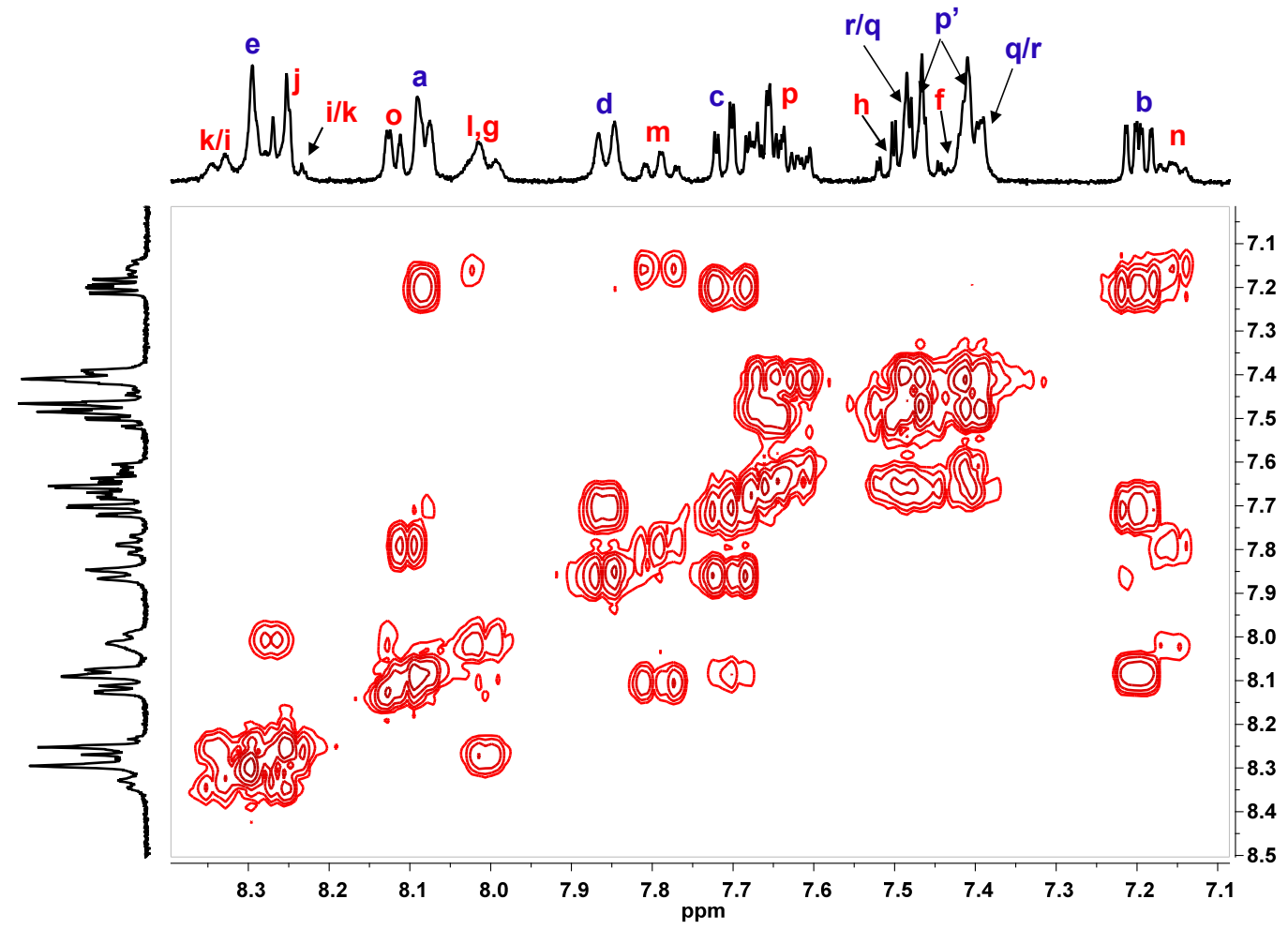

Figure S6. ${ }^{1} \mathrm{H}-{ }^{1} \mathrm{H}$ COSY spectrum of complex $[\mathrm{Ag}(\mathbf{1})]^{+}$in $\mathrm{CD}_{2} \mathrm{Cl}_{2}(400 \mathrm{MHz}, 298 \mathrm{~K})$.

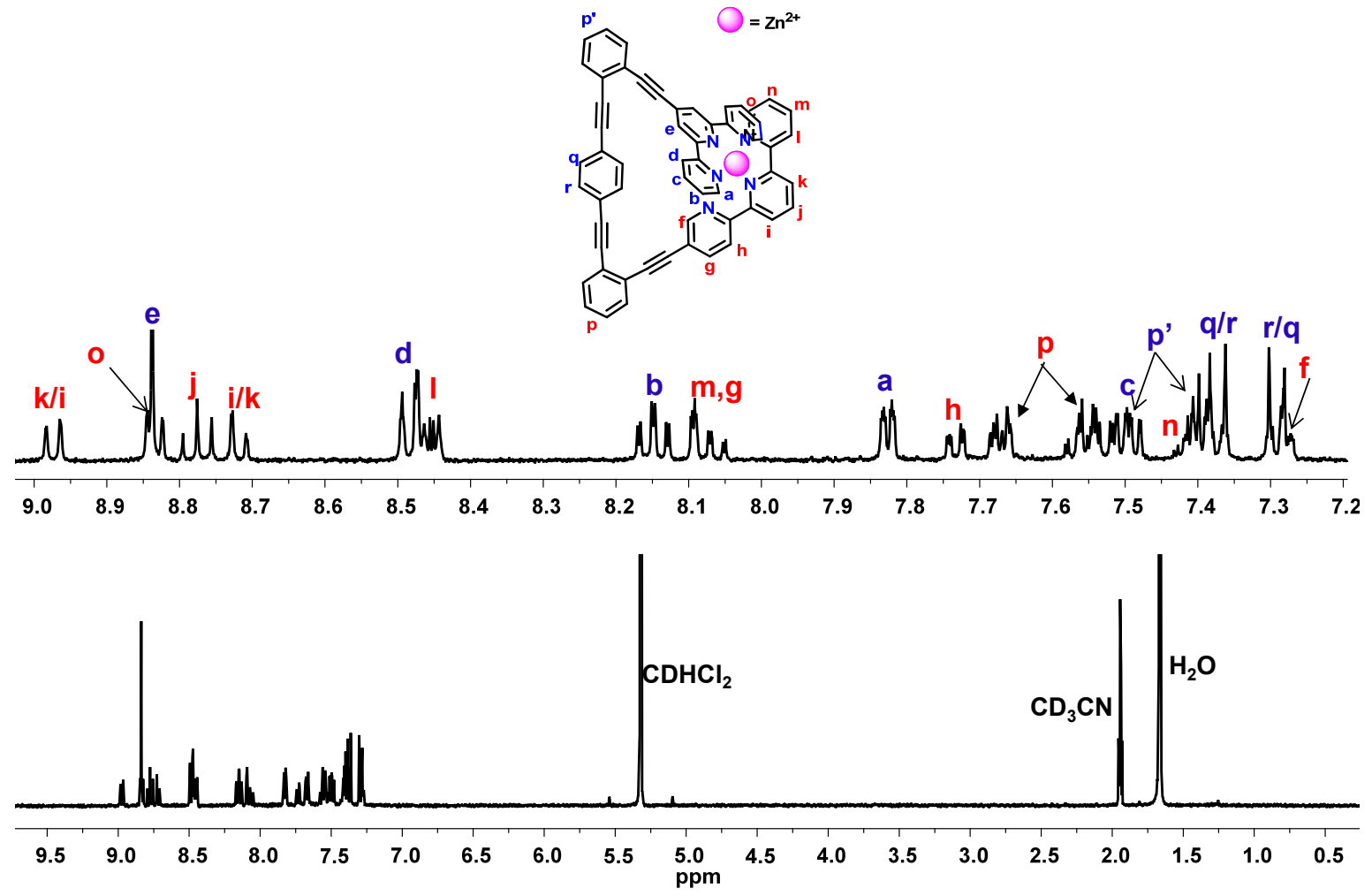

Figure S7. ${ }^{1} \mathrm{H}$ NMR spectrum of complex $[\mathrm{Zn}(\mathbf{1})]^{2+}$ in $\mathrm{CD}_{2} \mathrm{Cl}_{2}(400 \mathrm{MHz}, 298 \mathrm{~K})$. 


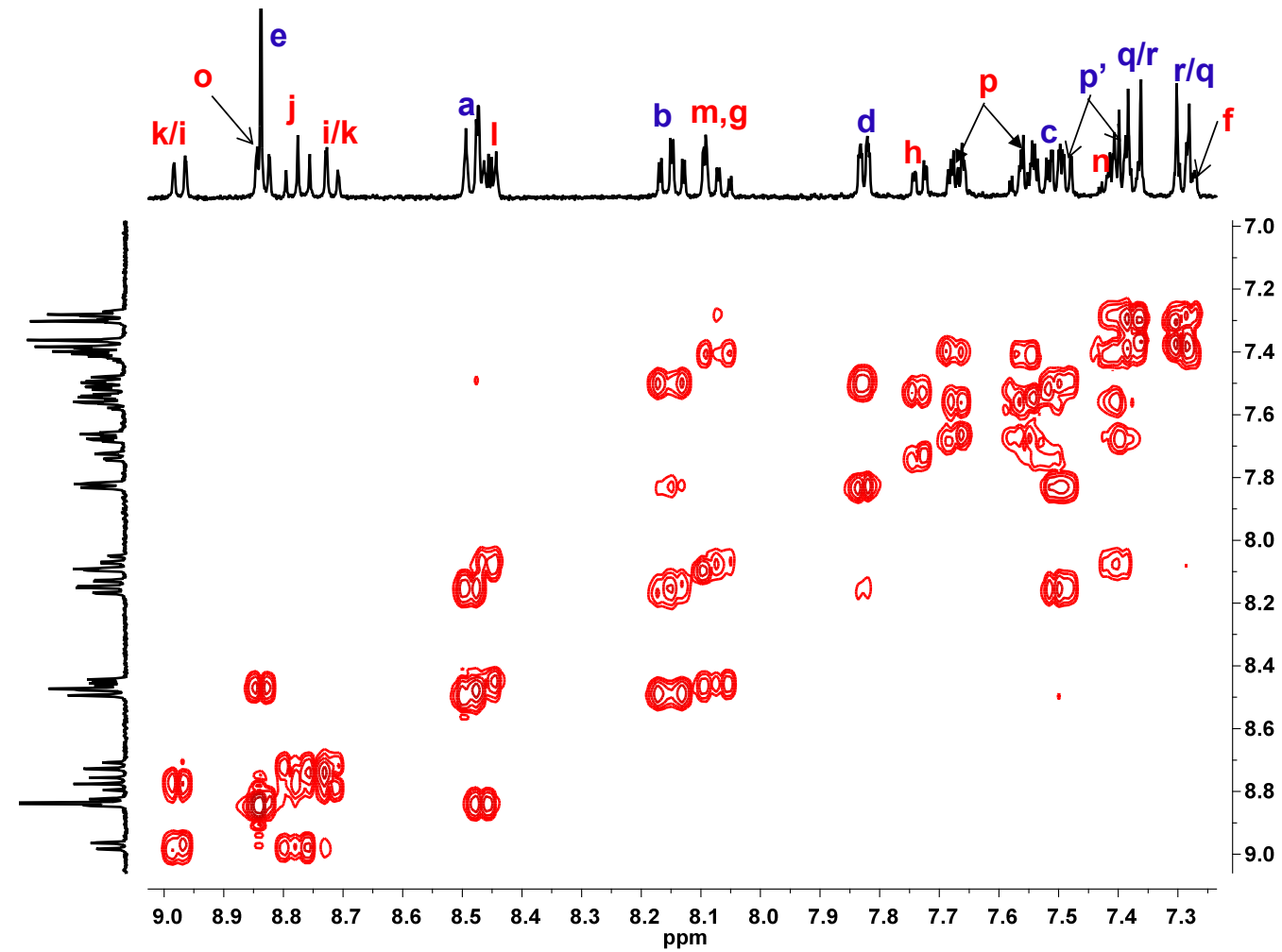

Figure S8. ${ }^{1} \mathrm{H}-{ }^{1} \mathrm{H}$ COSY spectrum of complex $[\mathrm{Zn}(\mathbf{1})]^{2+}$ in $\mathrm{CD}_{2} \mathrm{Cl}_{2}(400 \mathrm{MHz}, 298 \mathrm{~K})$.

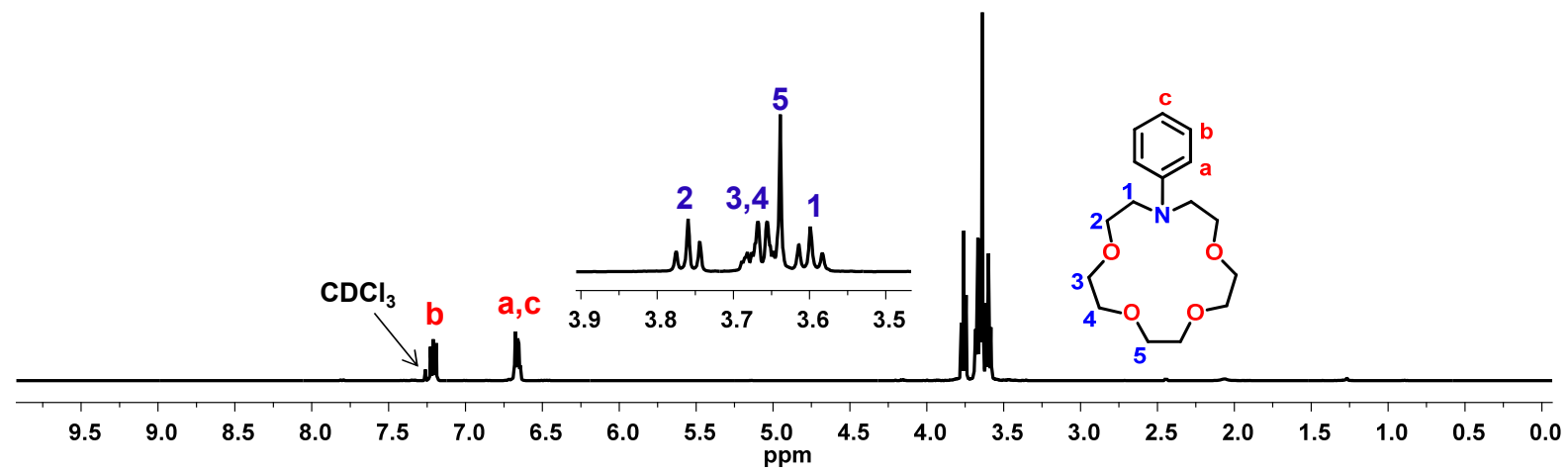

Figure S9. ${ }^{1} \mathrm{H}$ NMR spectrum of 21 in $\mathrm{CDCl}_{3}(400 \mathrm{MHz}, 298 \mathrm{~K})$. 


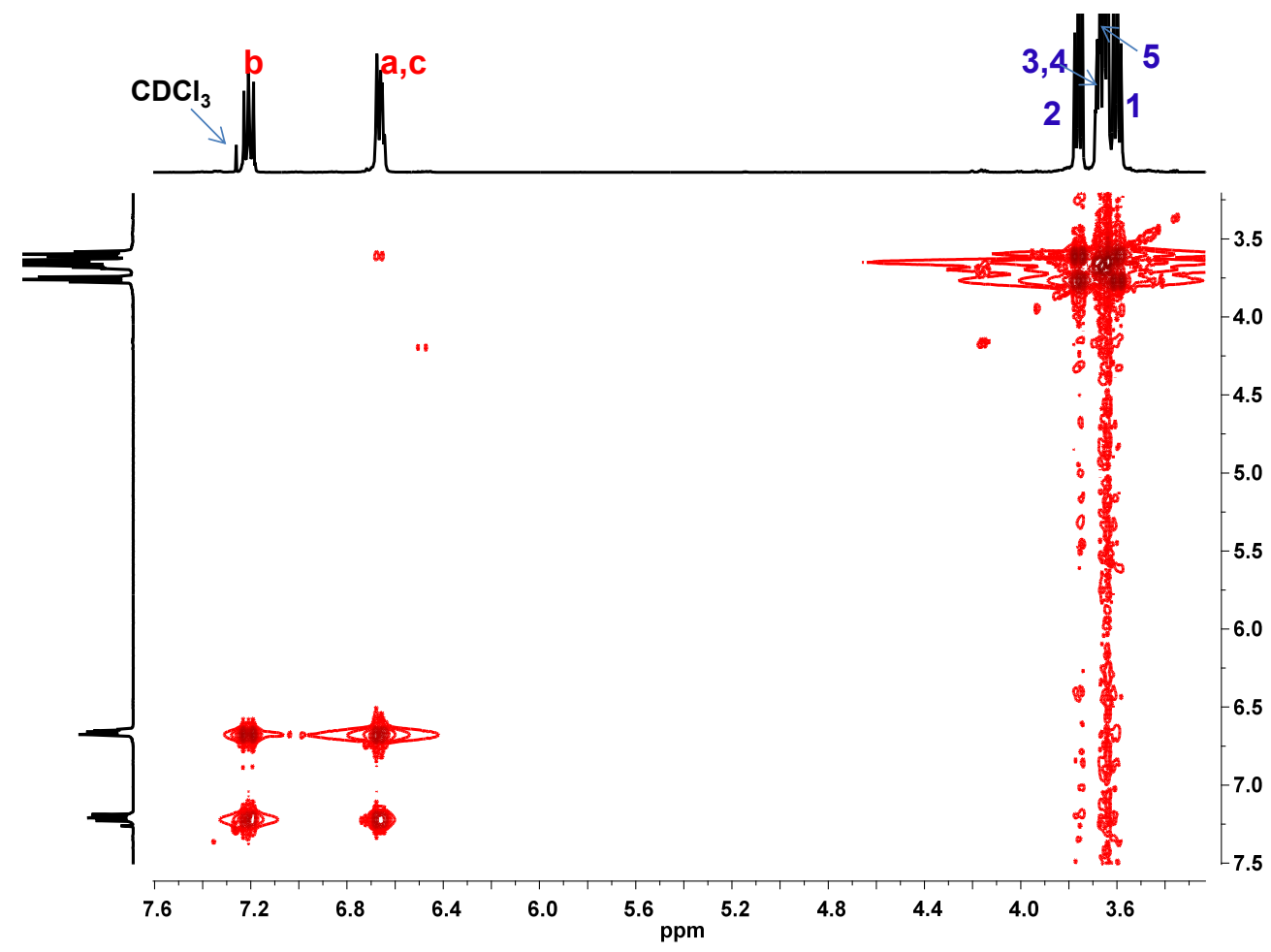

Figure S10. ${ }^{1} \mathrm{H}-{ }^{1} \mathrm{H}$ COSY spectrum of 21 in $\mathrm{CDCl}_{3}(400 \mathrm{MHz}, 298 \mathrm{~K})$.

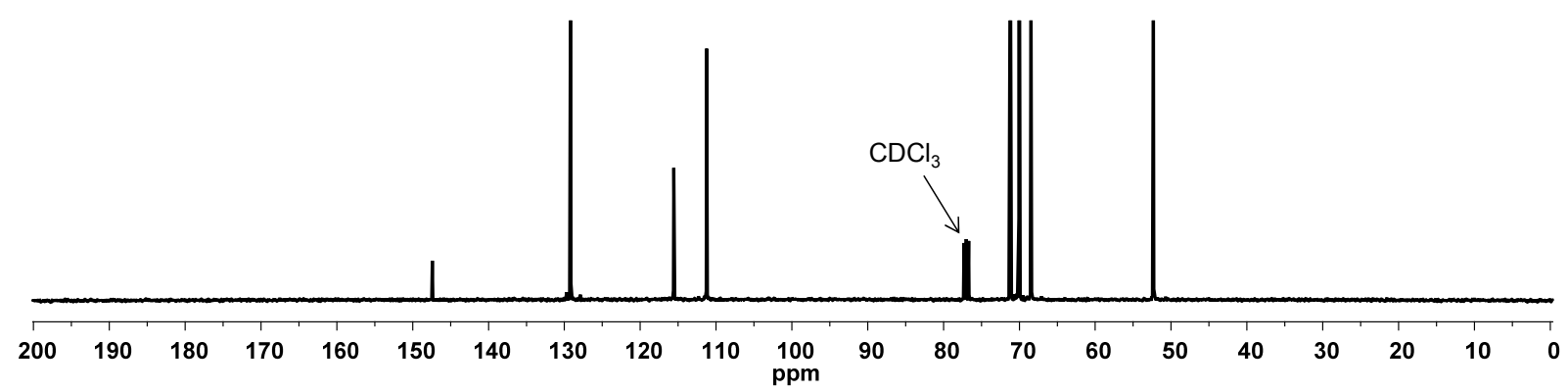

Figure S11. ${ }^{13} \mathrm{C}$ NMR spectrum of 21 in $\mathrm{CDCl}_{3}(100 \mathrm{MHz}, 298 \mathrm{~K})$. 


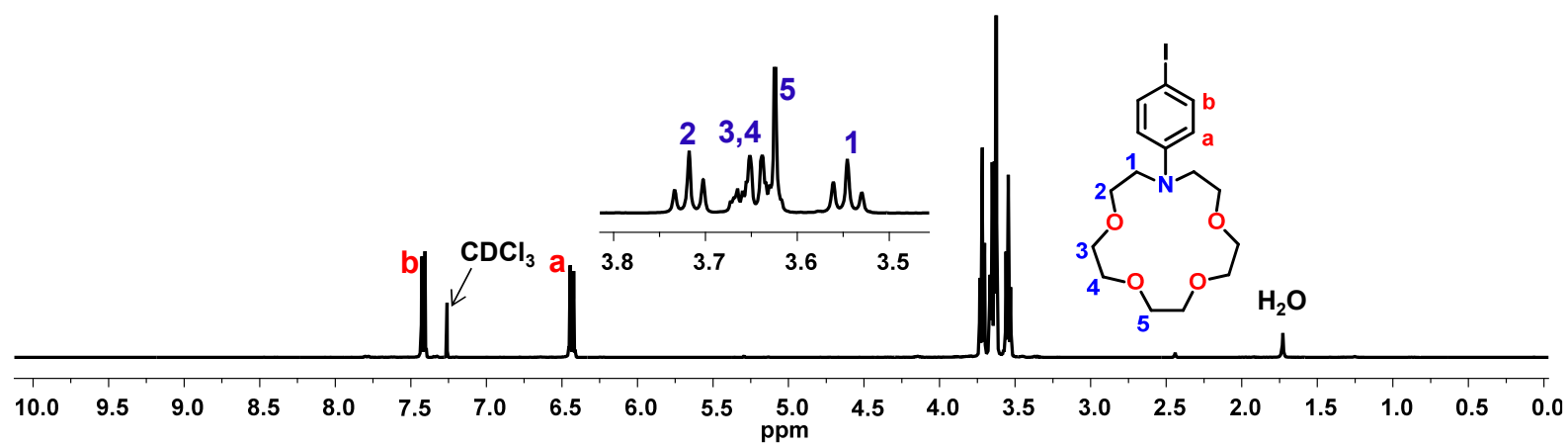

Figure S12. ${ }^{1} \mathrm{H}$ NMR spectrum of 22 in $\mathrm{CDCl}_{3}(400 \mathrm{MHz}, 298 \mathrm{~K})$.

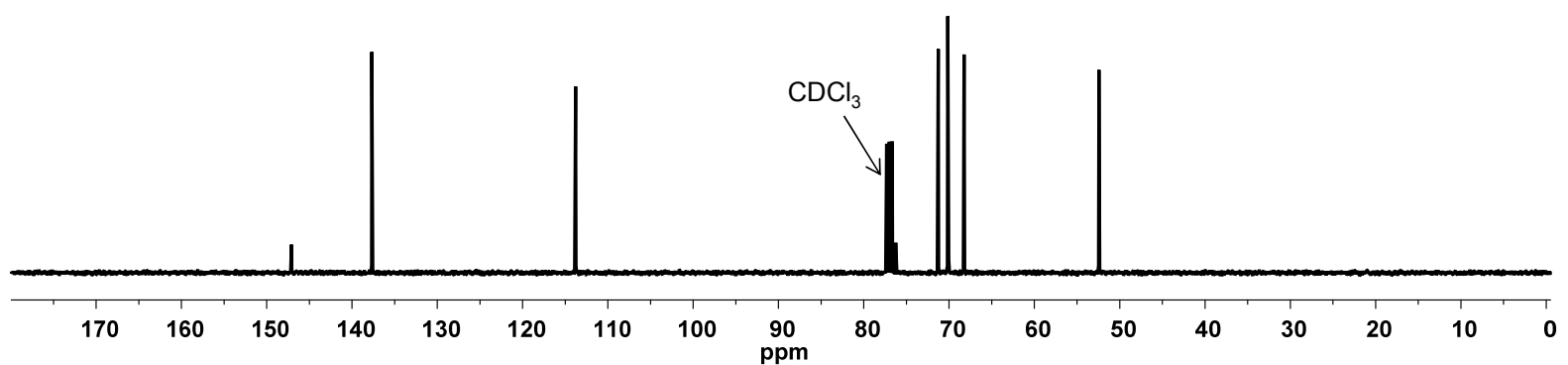

Figure S13. ${ }^{13} \mathrm{C}$ NMR spectrum of 22 in $\mathrm{CDCl}_{3}(100 \mathrm{MHz}, 298 \mathrm{~K})$.

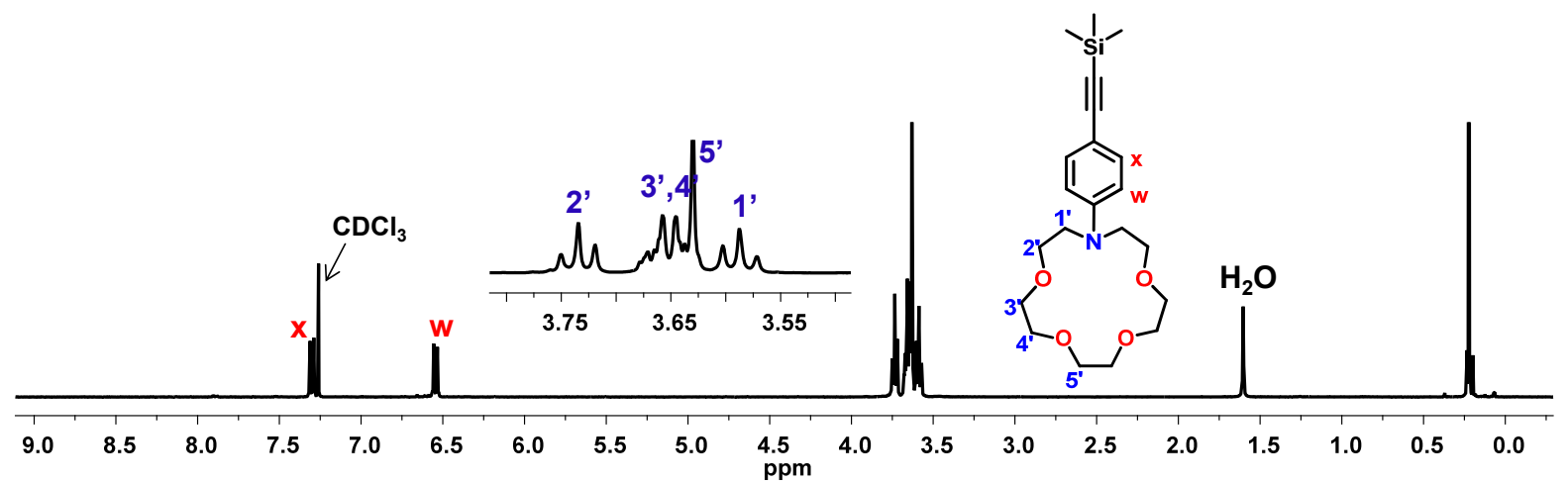

Figure S14. ${ }^{1} \mathrm{H}$ NMR spectrum of 4 in $\mathrm{CDCl}_{3}(400 \mathrm{MHz}, 298 \mathrm{~K})$. 


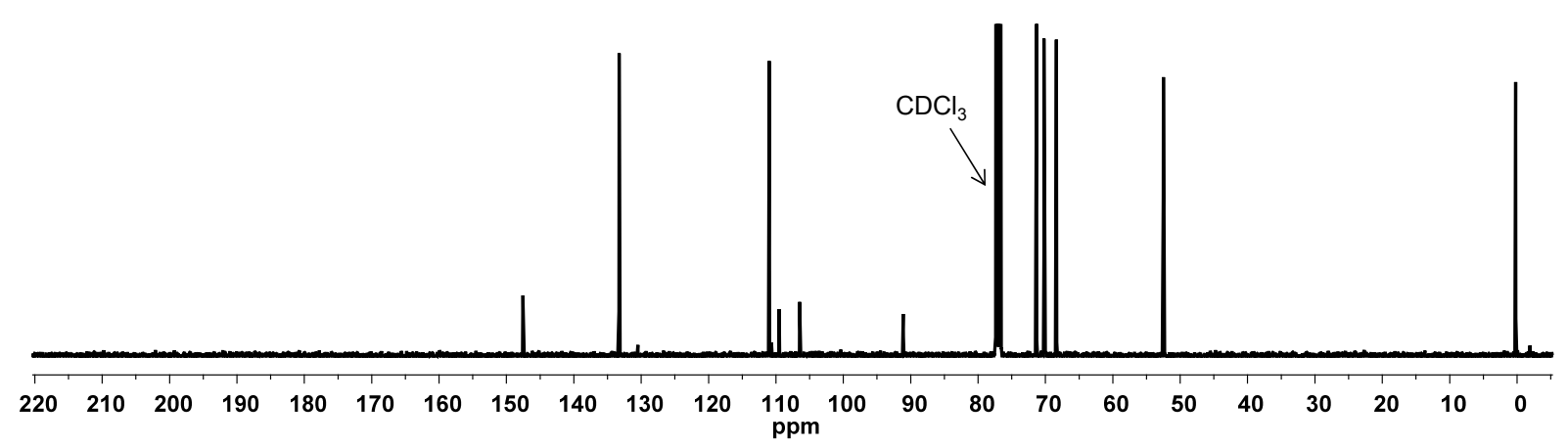

Figure S15. ${ }^{13} \mathrm{C}$ NMR spectrum of 4 in $\mathrm{CDCl}_{3}(100 \mathrm{MHz}, 298 \mathrm{~K})$.

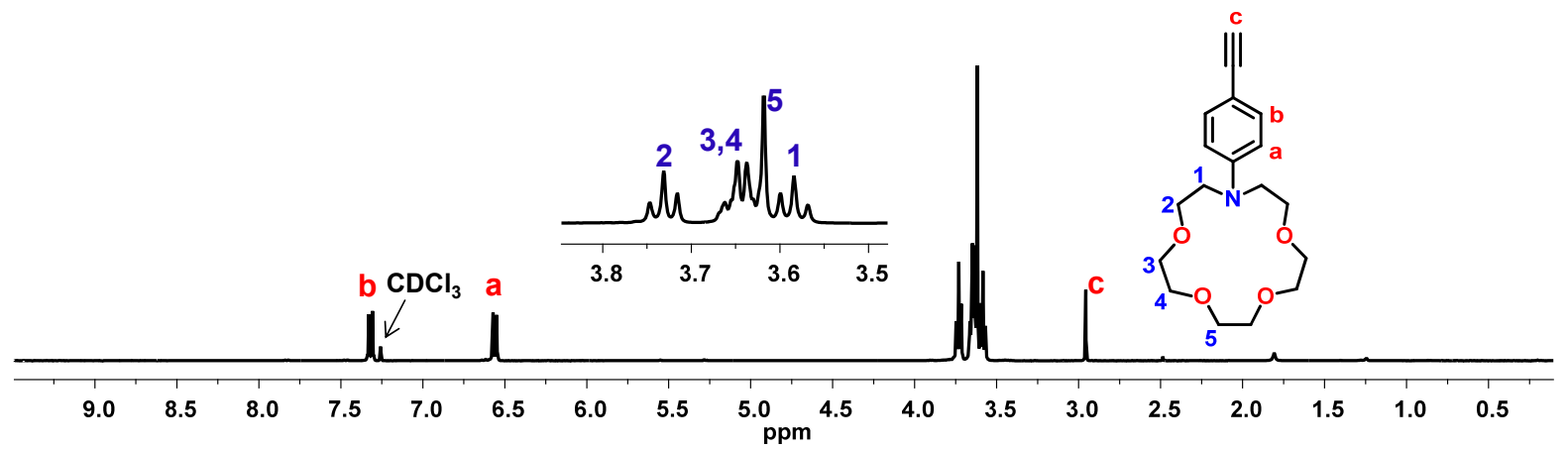

Figure S16. ${ }^{1} \mathrm{H}$ NMR spectrum of 23 in $\mathrm{CDCl}_{3}(400 \mathrm{MHz}, 298 \mathrm{~K})$.

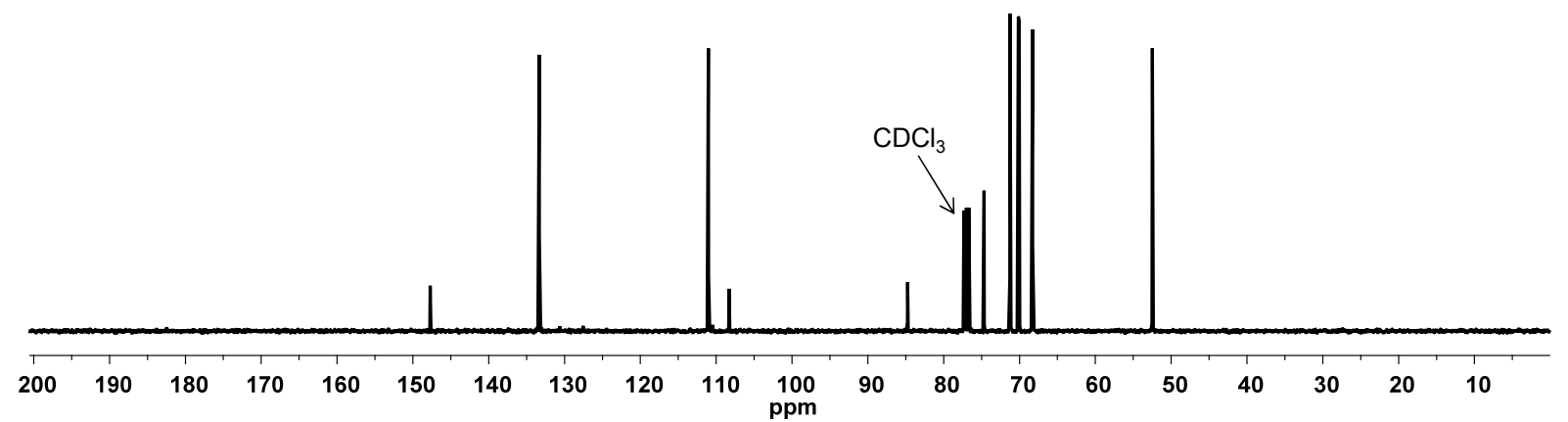

Figure S17. ${ }^{13} \mathrm{C}$ NMR spectrum of 23 in $\mathrm{CDCl}_{3}(100 \mathrm{MHz}, 298 \mathrm{~K})$. 


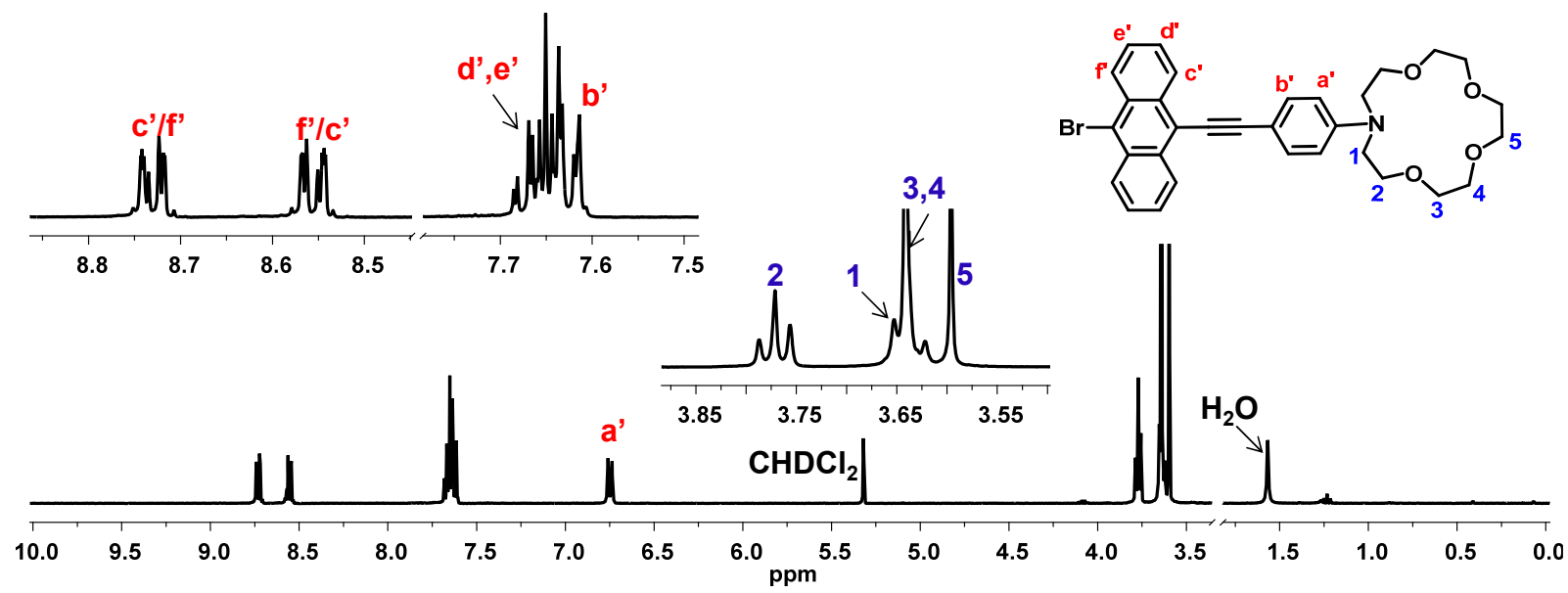

Figure S18. ${ }^{1} \mathrm{H}$ NMR spectrum of 2 in $\mathrm{CD}_{2} \mathrm{Cl}_{2}(400 \mathrm{MHz}, 298 \mathrm{~K})$.

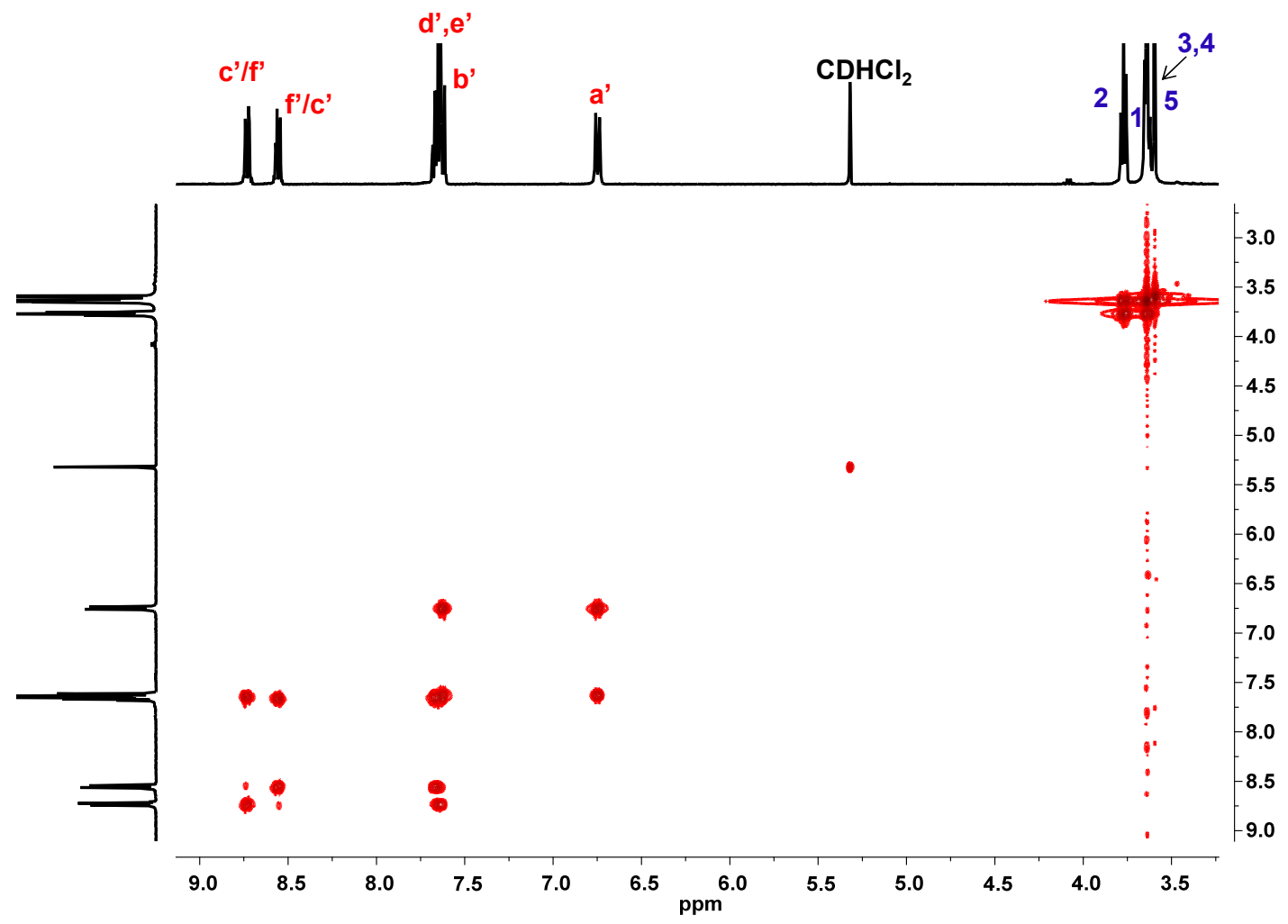

Figure S19. ${ }^{1} \mathrm{H}-{ }^{1} \mathrm{H}$ COSY spectrum of 2 in $\mathrm{CD}_{2} \mathrm{Cl}_{2}(400 \mathrm{MHz}, 298 \mathrm{~K})$. 


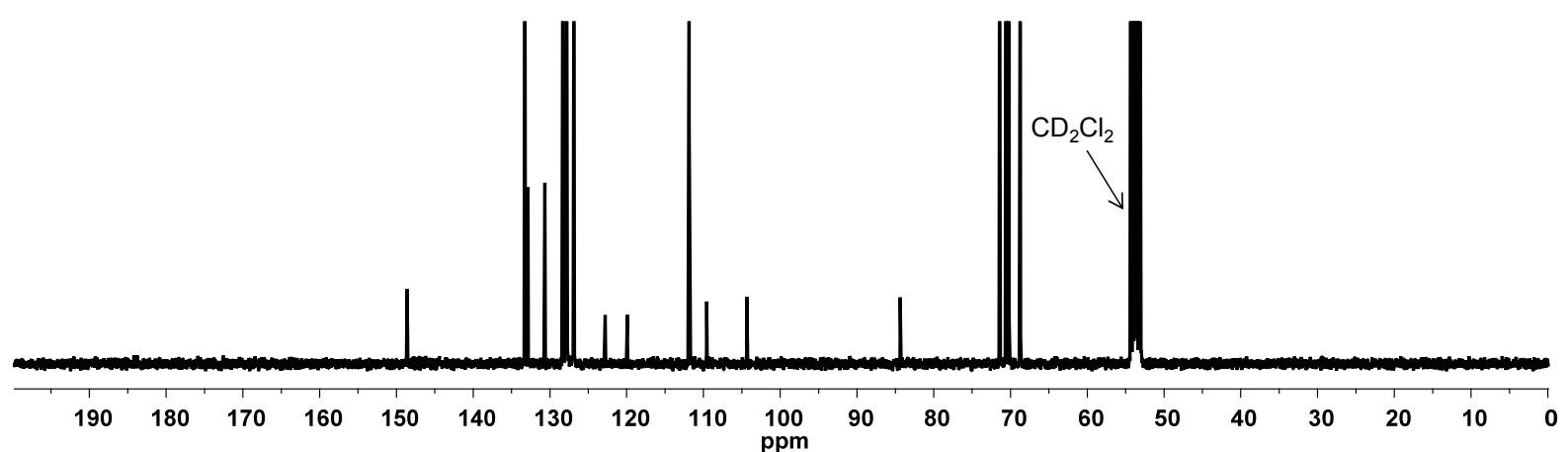

Figure S20. ${ }^{13} \mathrm{C}$ NMR spectrum of 2 in $\mathrm{CD}_{2} \mathrm{Cl}_{2}(100 \mathrm{MHz}, 298 \mathrm{~K})$.

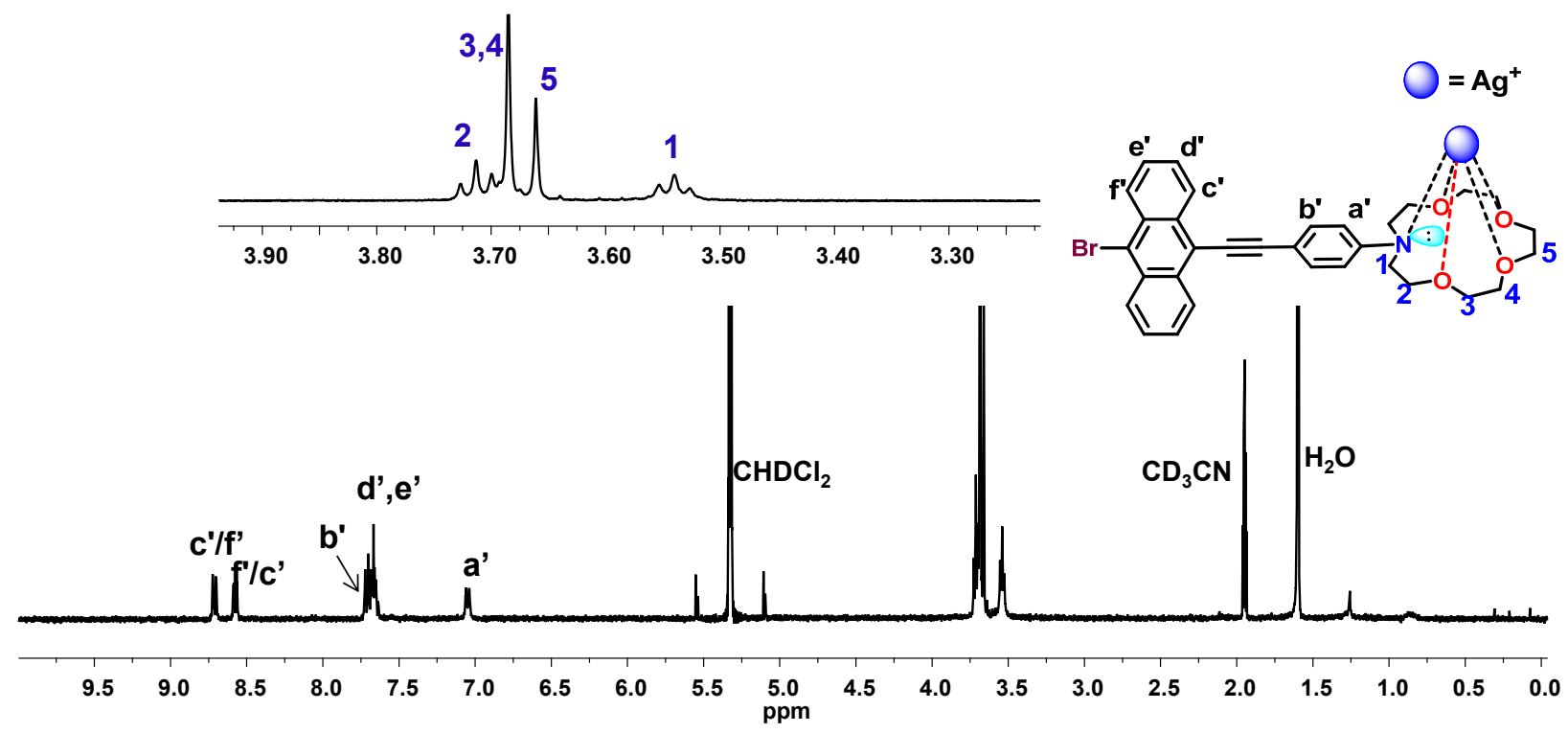

Figure S21. ${ }^{1} \mathrm{H}$ NMR spectrum of complex $[\mathrm{Ag}(\mathbf{2})]^{+}$in $\mathrm{CD}_{2} \mathrm{Cl}_{2}(400 \mathrm{MHz}, 298 \mathrm{~K})$. 


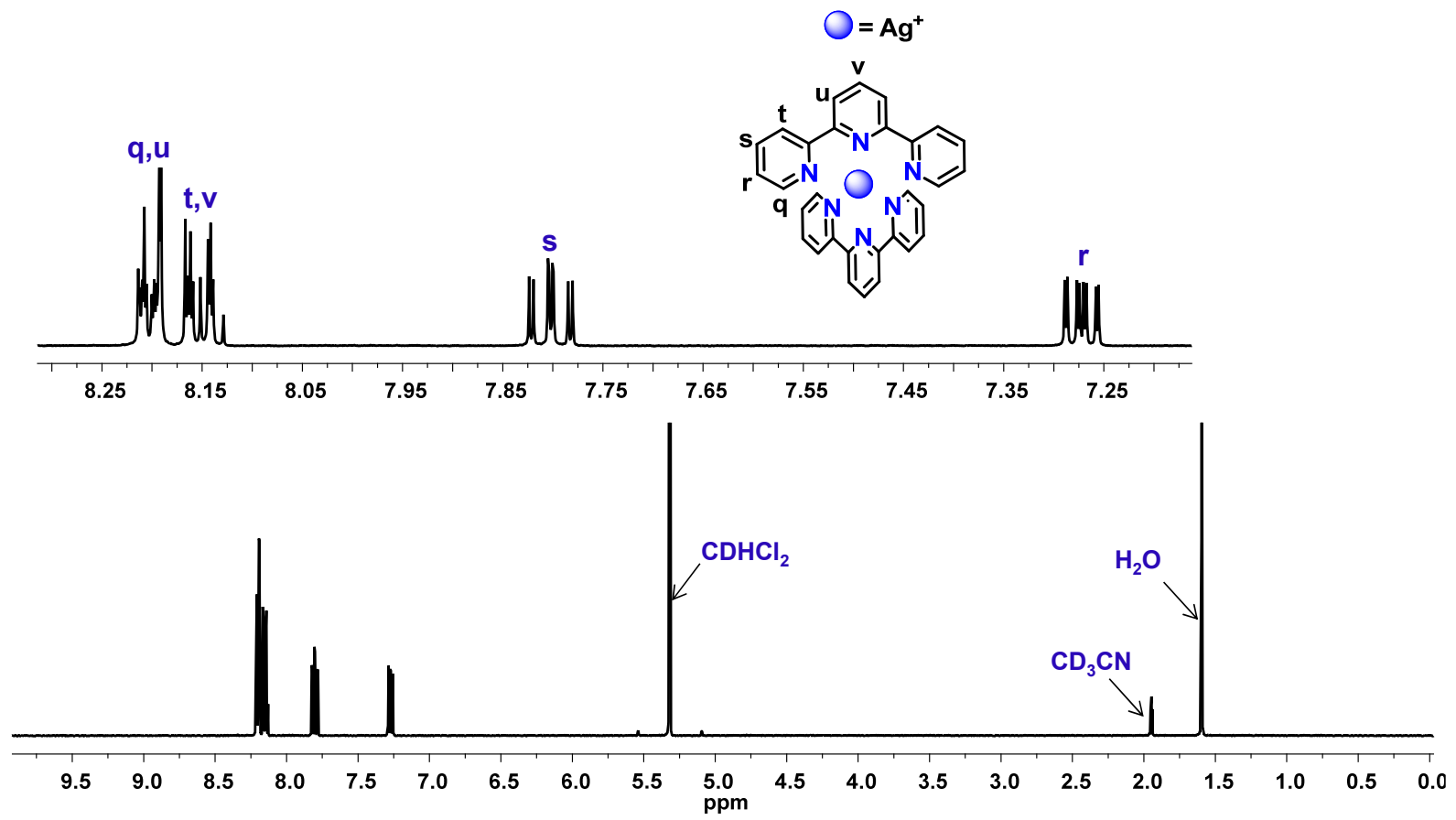

Figure S22. ${ }^{1} \mathrm{H}$ NMR spectrum of complex 5 in $\mathrm{CD}_{2} \mathrm{Cl}_{2}(400 \mathrm{MHz}, 298 \mathrm{~K})$.

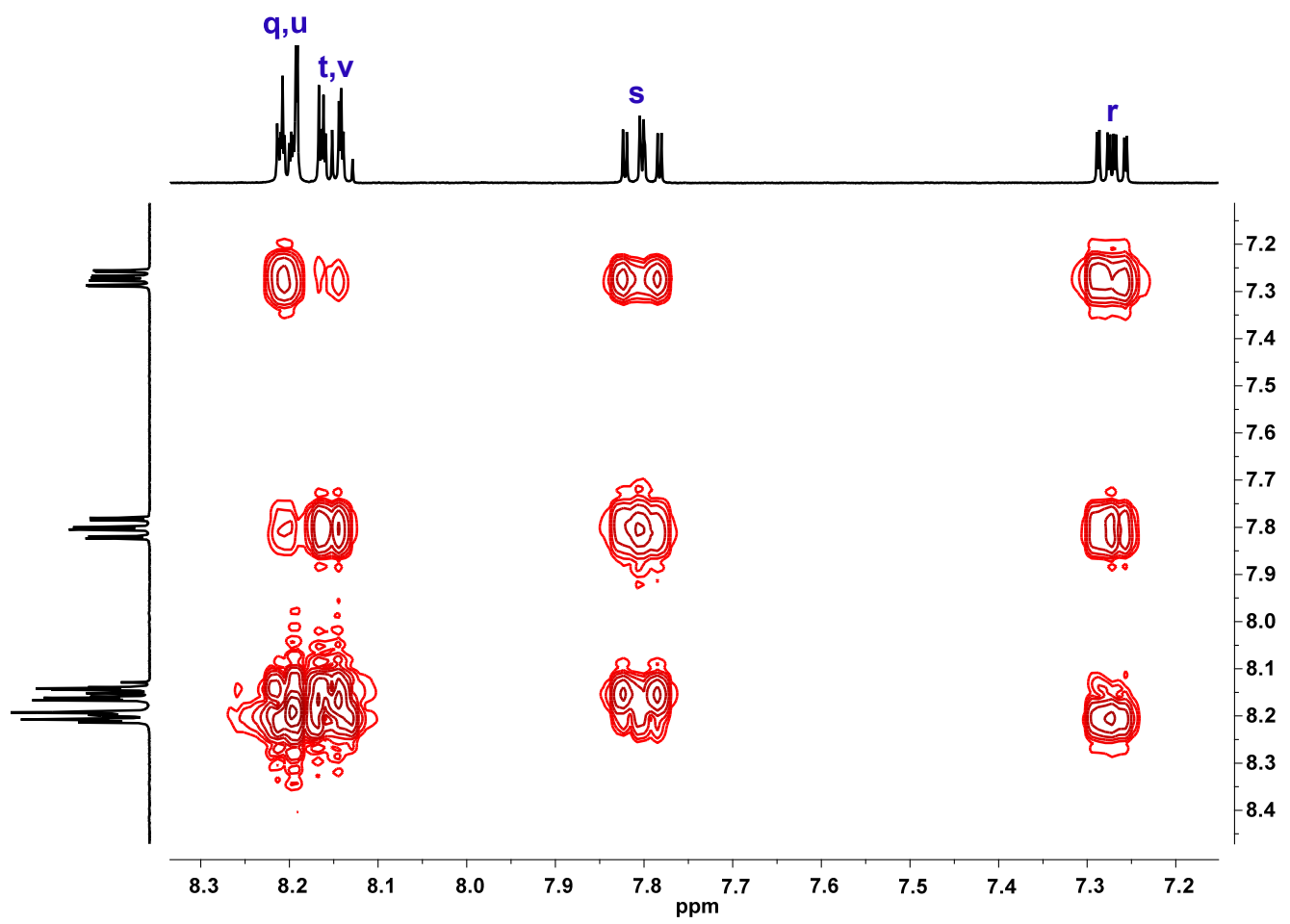

Figure S23. ${ }^{1} \mathrm{H}-{ }^{1} \mathrm{H}$ COSY spectrum of complex 5 in $\mathrm{CD}_{2} \mathrm{Cl}_{2}(400 \mathrm{MHz}, 298 \mathrm{~K})$. 


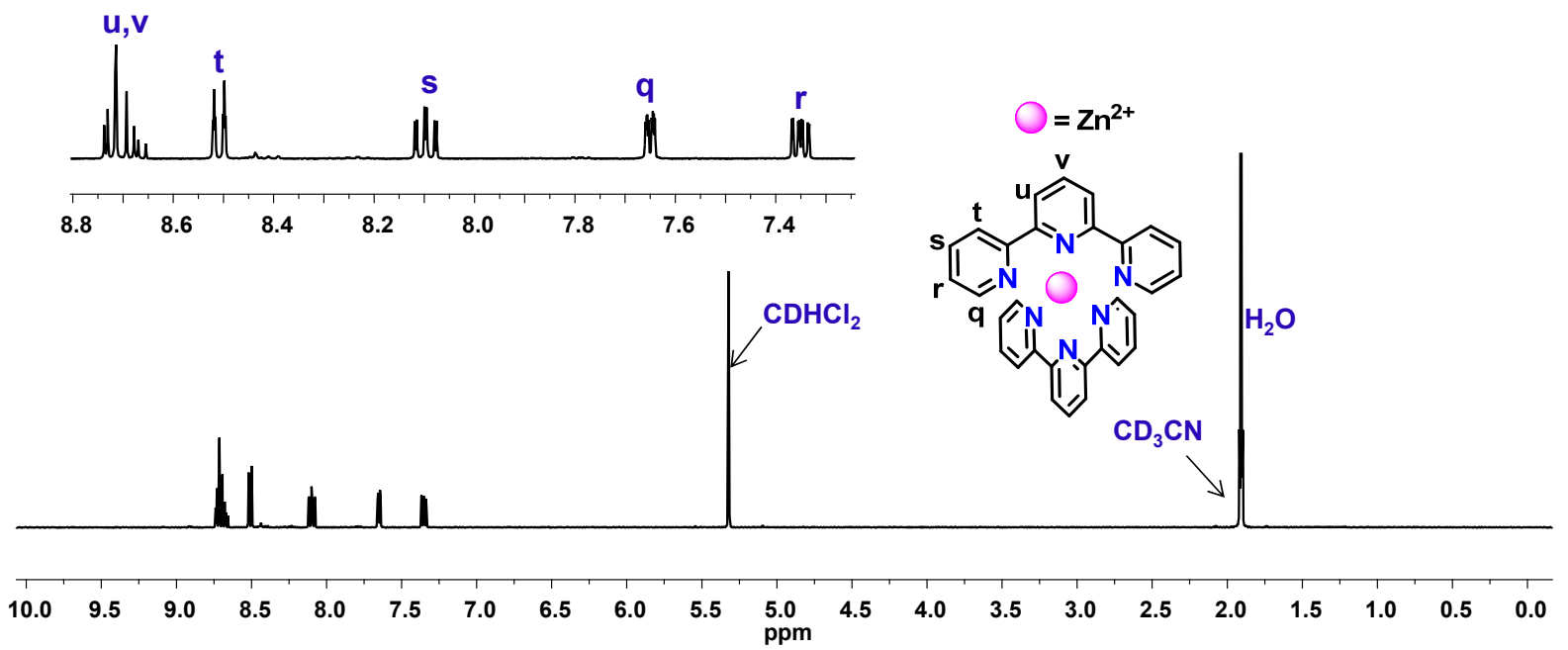

Figure S24. ${ }^{1} \mathrm{H}$ NMR spectrum of complex 6 in $\mathrm{CD}_{2} \mathrm{Cl}_{2}(400 \mathrm{MHz}, 298 \mathrm{~K})$.

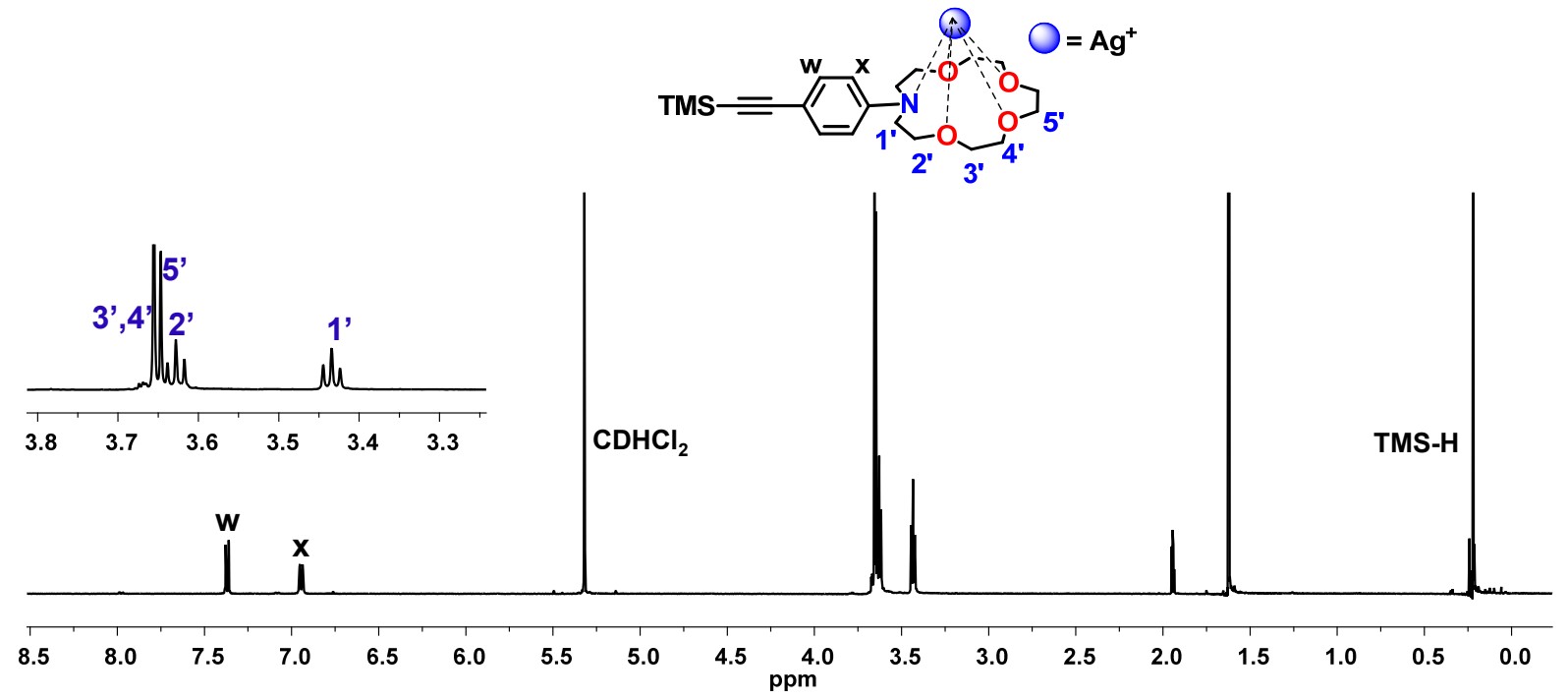

Figure S25. ${ }^{1} \mathrm{H}$ NMR spectrum of complex 7 in $\mathrm{CD}_{2} \mathrm{Cl}_{2}(400 \mathrm{MHz}, 298 \mathrm{~K})$. 

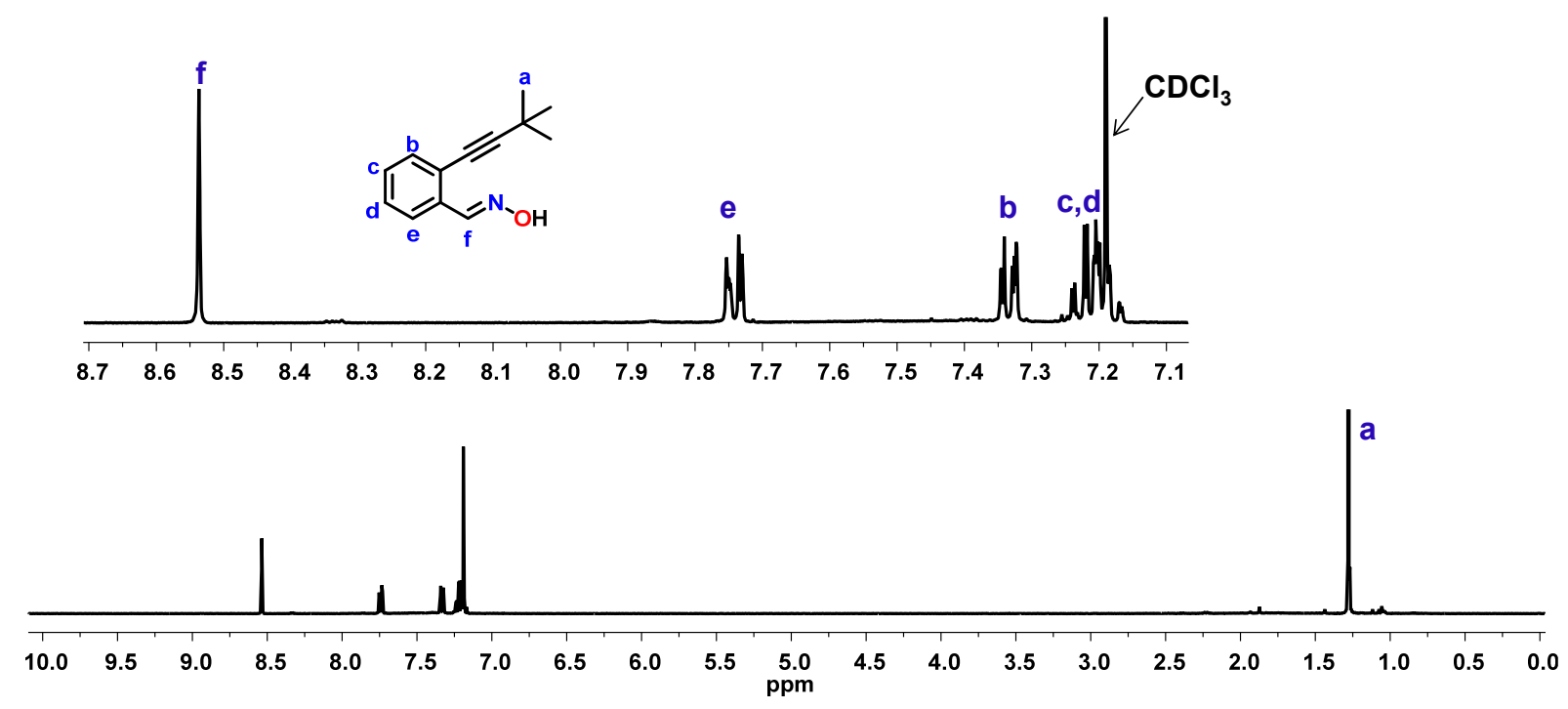

Figure S26. ${ }^{1} \mathrm{H}$ NMR spectrum of $\mathbf{A}$ in $\mathrm{CDCl}_{3}(400 \mathrm{MHz}, 298 \mathrm{~K})$.

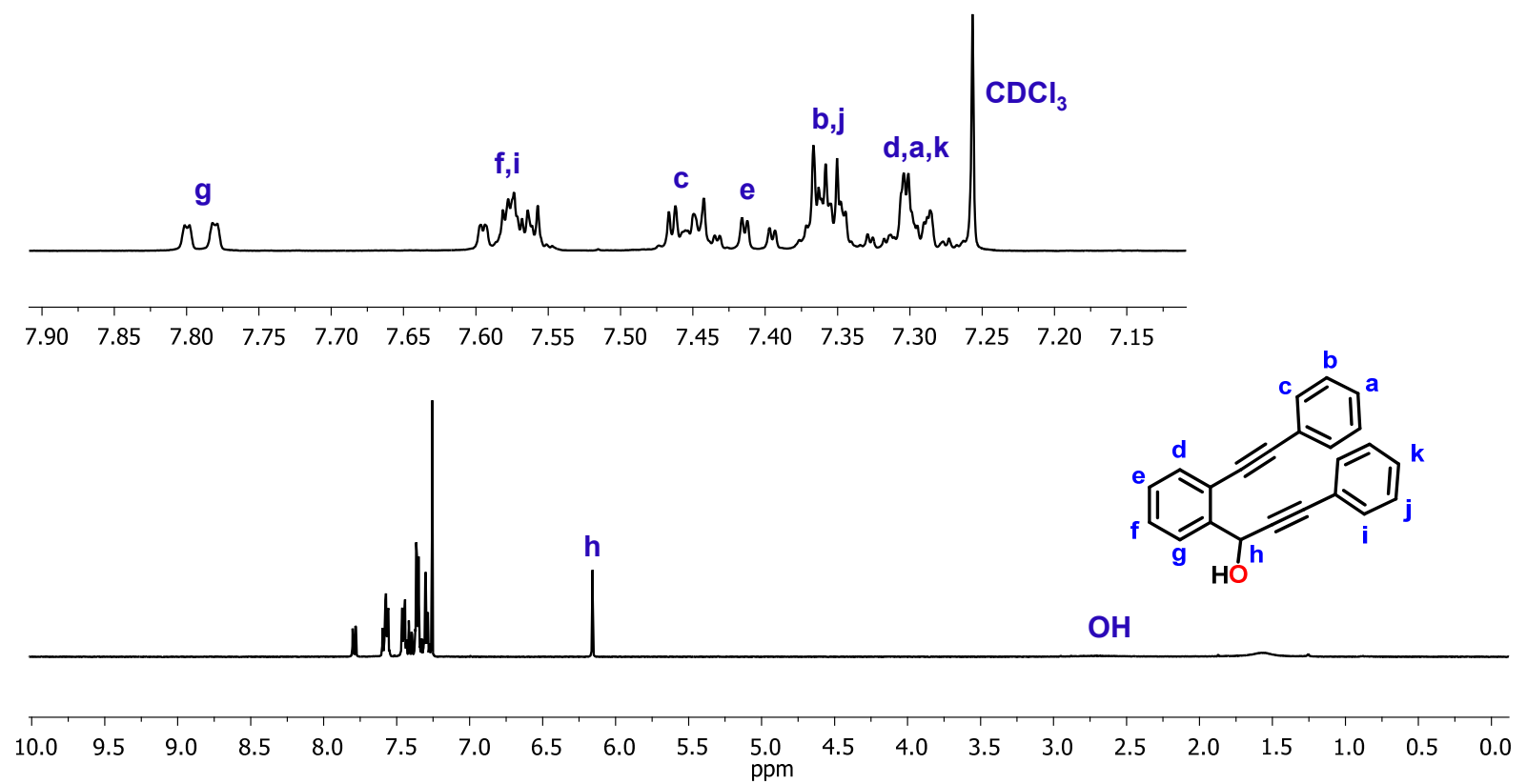

Figure S27. ${ }^{1} \mathrm{H}$ NMR spectrum of $\mathbf{C}$ in $\mathrm{CDCl}_{3}(400 \mathrm{MHz}, 298 \mathrm{~K})$. 

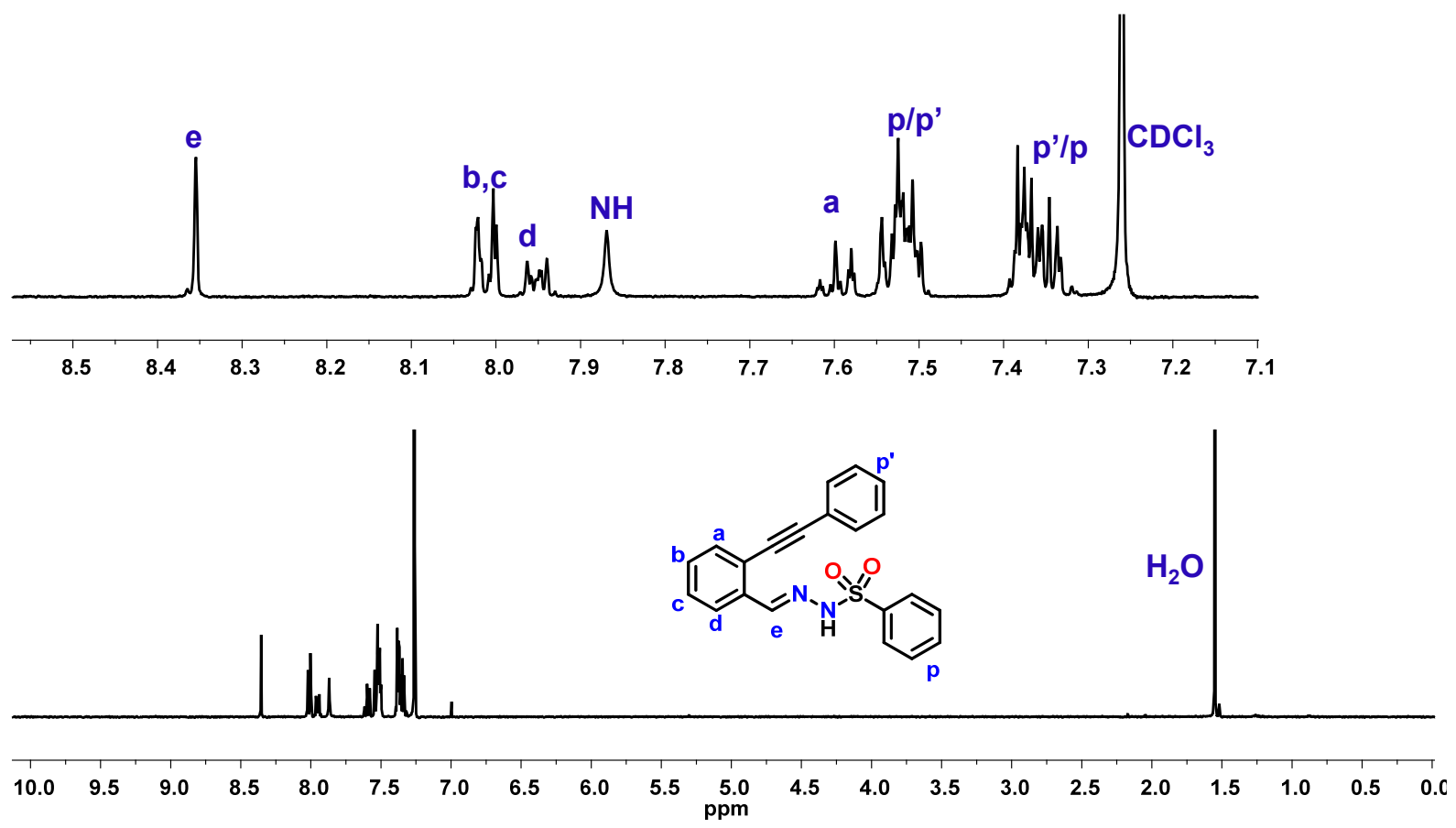

Figure S28. ${ }^{1} \mathrm{H}$ NMR spectrum of $\mathbf{E}$ in $\mathrm{CDCl}_{3}(400 \mathrm{MHz}, 298 \mathrm{~K})$.

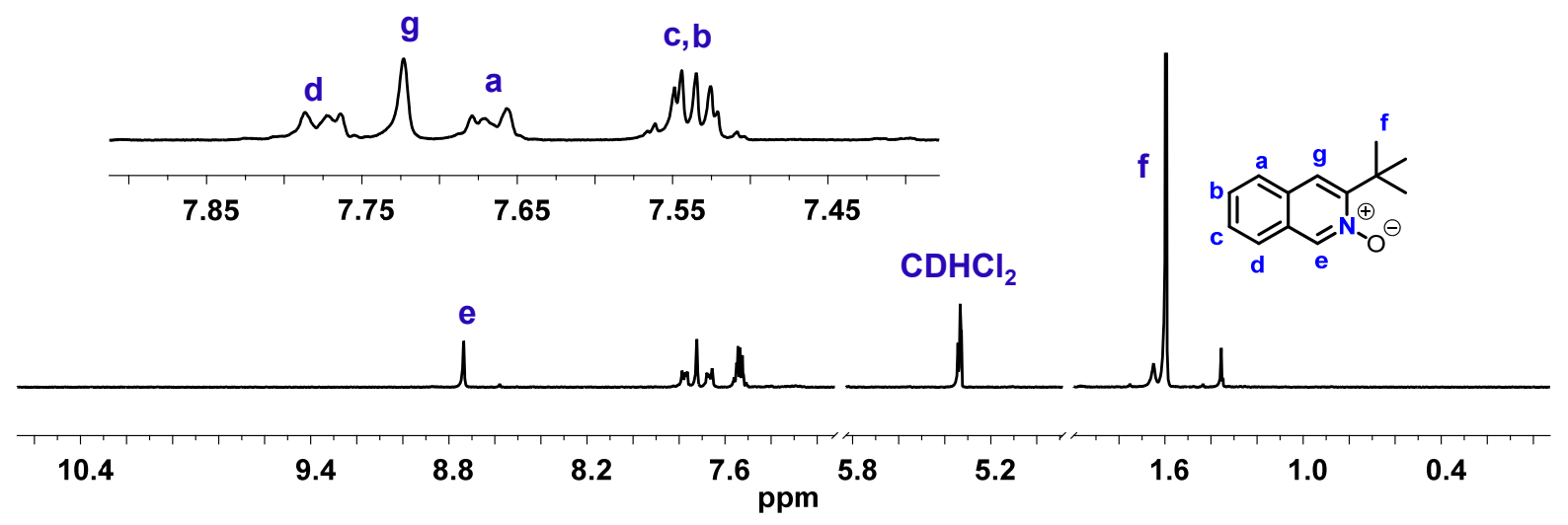

Figure S29. ${ }^{1} \mathrm{H}$ NMR spectrum of $\mathbf{B}$ in $\mathrm{CD}_{2} \mathrm{Cl}_{2}(400 \mathrm{MHz}, 298 \mathrm{~K})$. 


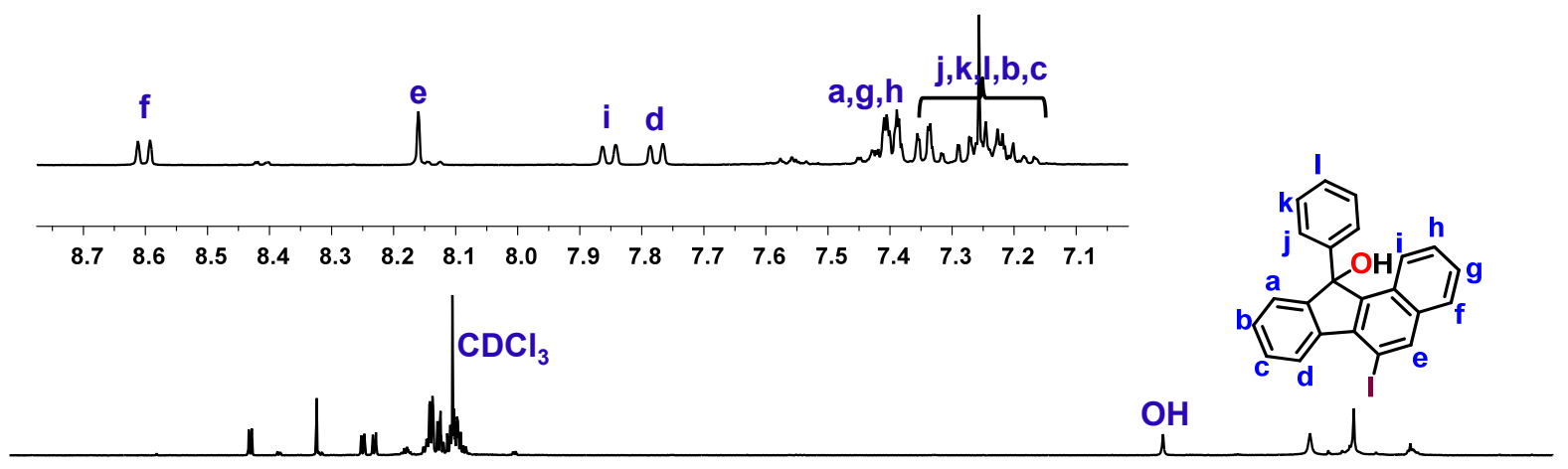

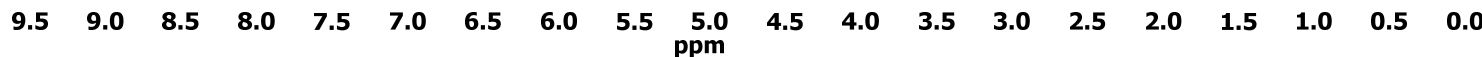

Figure S30. ${ }^{1} \mathrm{H}$ NMR spectrum of $\mathbf{D}$ in $\mathrm{CDCl}_{3}(400 \mathrm{MHz}, 298 \mathrm{~K})$.

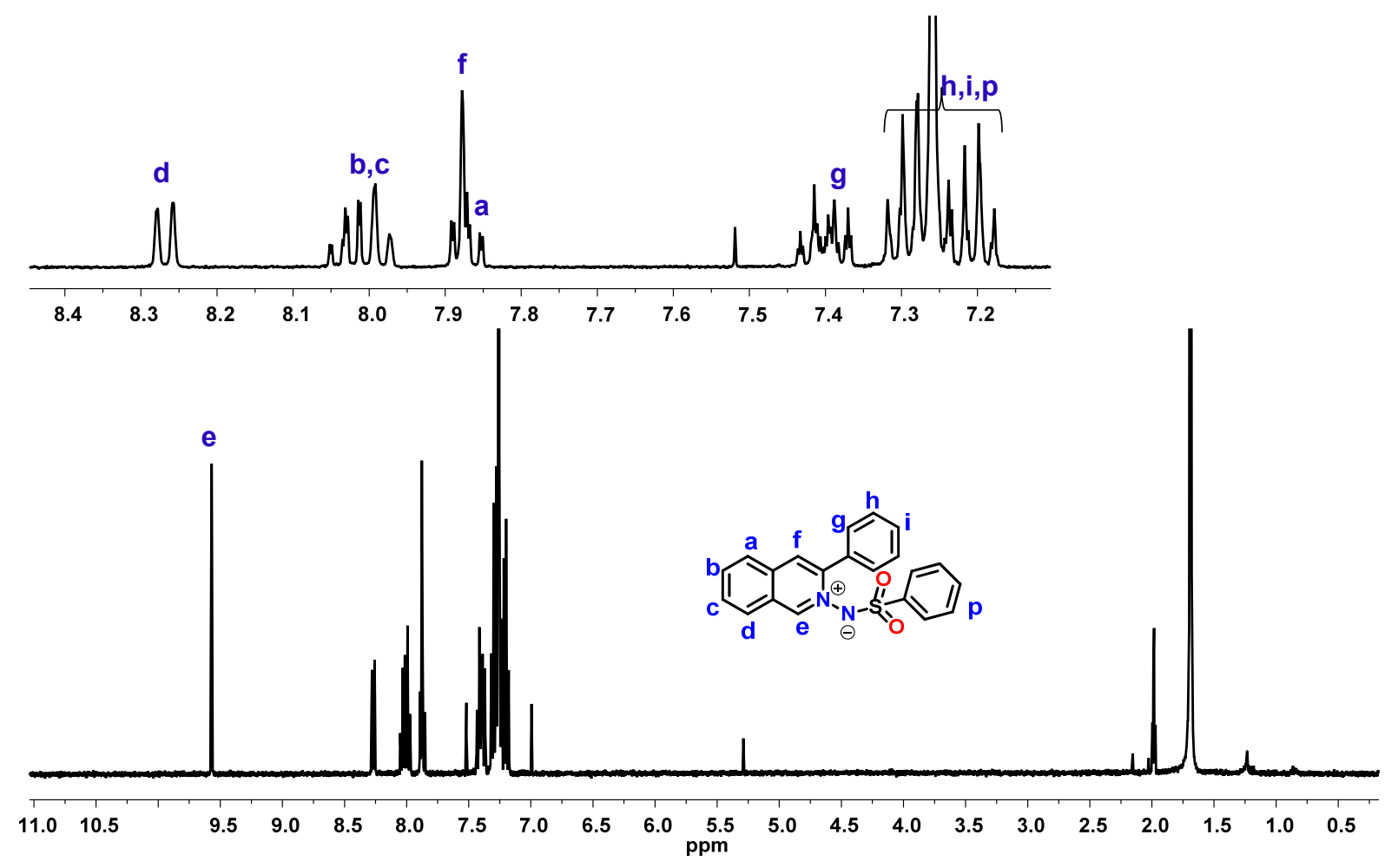

Figure S31. ${ }^{1} \mathrm{H}$ NMR spectrum of $\mathbf{F}$ in $\mathrm{CDCl}_{3}(400 \mathrm{MHz}, 298 \mathrm{~K})$. 


\section{Catalytic Experiments}

\section{General procedure}

All catalytic reactions were performed in $\mathrm{CD}_{2} \mathrm{Cl}_{2}: \mathrm{CD}_{3} \mathrm{CN}=50: 1$ and in an NMR tube. The mixture was heated at $40{ }^{\circ} \mathrm{C}$ for $2 \mathrm{~h}$ in a thermostat. Solids were transferred first, followed by addition of the solvent. Finally liquids were added (as standard solutions in $\mathrm{CDCl}_{2}$ ). Product yields were determined by using 1,1,2,2-tetrachloroethane (TCE) as an internal standard.

\section{Synthesis of compound $B^{4}$}

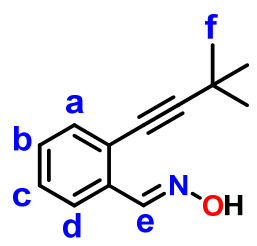

A

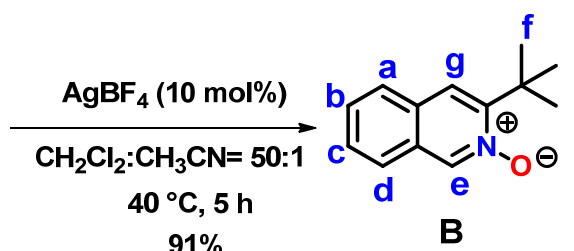

91\%

Compound A (100 mg, $496 \mu \mathrm{mol})$ was dissolved in $5 \mathrm{~mL}$ of DCM, then $\mathrm{AgBF}_{4}(8.00 \mathrm{mg}, 49.6$ $\mu \mathrm{mol}, 10 \mathrm{~mol} \%$ ) in $100 \mu \mathrm{L}$ of acetonitrile was added, and the mixture was heated for $5 \mathrm{~h}$ at 40 ${ }^{\circ} \mathrm{C}$. The solvent was removed and the residue was purified by chromatography (silica gel, $\left.\mathrm{MeOH} / \mathrm{DCM}=1: 10, R_{f}=0.4\right)$ to furnish product $\mathbf{B}(91 \mathrm{mg}, 451 \mu \mathrm{mol}, 91 \%)$ as a white solid. IR (KBr): $\tilde{v}=488,505,559,570,631,660,709,762,796,863,911,915,940,961,1001,1177$, $1219,1280,1301,1369,1385,1442,1478,1488,1591,1932,2232,2869,2921,2967,3001 \mathrm{~cm}^{-}$ 1. ${ }^{1} \mathbf{H}$ NMR (400 MHz, $\left.\mathbf{C D}_{2} \mathbf{C l}_{2}\right): \delta=1.60(\mathrm{~s}, 9 \mathrm{H}, \mathrm{f}-\mathrm{H}), 7.50-7.58(\mathrm{~m}, 2 \mathrm{H}, \mathrm{c}-, \mathrm{b}-\mathrm{H}), 7.65-7.69(\mathrm{~m}$, $1 \mathrm{H}, \mathrm{a}-\mathrm{H}), 7.73(\mathrm{~s}, 1 \mathrm{H}, \mathrm{e}-\mathrm{H}), 7.75-7.8(\mathrm{~m}, 1 \mathrm{H}, \mathrm{d}-\mathrm{H}) \mathrm{ppm}$. Elemental analysis: Calcd. for $\mathrm{C}_{13} \mathrm{H}_{15} \mathrm{NO}$ : C, 77.58; H, 7.51; N, 6.96. Found: C, 77.31; H, 7.46; N, 6.71.

\section{Synthesis of compound $D^{7}$}

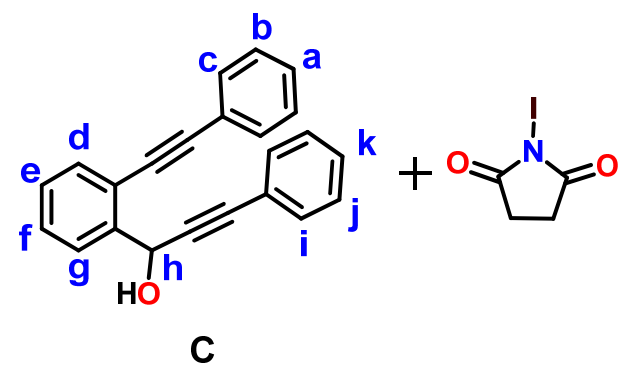

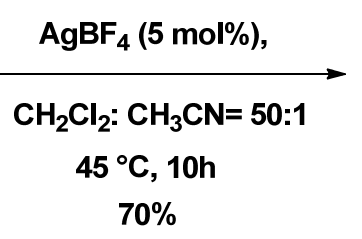

$70 \%$

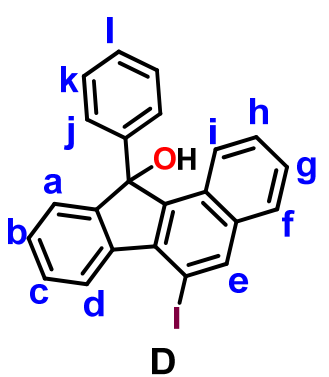

D 
Compound C (100 mg, $324 \mu \mathrm{mol})$ and NIS ( $87.5 \mathrm{mg}, 389 \mu \mathrm{mol}, 1.2$ eqv.) were dissolved in 5 $\mathrm{mL}$ of dichloromethane. Then $\mathrm{AgBF}_{4}(3.10 \mathrm{mg}, 16.2 \mu \mathrm{mol}, 5 \mathrm{~mol} \%)$ in $100 \mu \mathrm{L}$ of acetonitrile was added and the mixture was heated for $10 \mathrm{~h}$ at $45{ }^{\circ} \mathrm{C}$. The solvent was removed and the residue was purified by chromatography ( silica gel, $\mathrm{DCM} /$ hexane $=1: 1, R_{f}=0.5$ ) to give product D (98 mg, $226 \mu \mathrm{mol}, 70 \%)$ as a yellow solid. IR (KBr): $\widetilde{v}=520,541,550,574,624,620,735$, 801, 822, 905, 973, 990, 1021, 1029, 1086, 1149, 1185, 1278, 1310, 1383, 1422, 1490, 1560, 1591, 1620, 1690, $3050 \mathrm{~cm}^{-1}$. ${ }^{1}$ H NMR (400 MHz, $\left.\mathbf{C D C l}_{3}\right): \delta=2.5$ (brs, 1H, OH), 7.15-7.36 (m, 7H, j-, k-, l-, b-, c-H), 7.36-7.45 (m, 3H, a-, g-, h-H), $7.78\left(\mathrm{dd},{ }^{3} J=8.0 \mathrm{~Hz},{ }^{4} J=1.0 \mathrm{~Hz}, 1 \mathrm{H}\right.$, d-H), $7.85\left(\mathrm{dd},{ }^{3} J=8.0 \mathrm{~Hz},{ }^{4} J=1.0 \mathrm{~Hz}, 1 \mathrm{H}, \mathrm{i}-\mathrm{H}\right), 8.16(\mathrm{~s}, 1 \mathrm{H}, \mathrm{e}-\mathrm{H}), 8.60\left(\mathrm{dd},{ }^{3} J=8.0 \mathrm{~Hz},{ }^{4} \mathrm{~J}=\right.$ $1.0 \mathrm{~Hz}, 1 \mathrm{H}, \mathrm{f}-\mathrm{H}) \mathrm{ppm}$.

\section{Synthesis of compound $\mathrm{F}^{8}$}

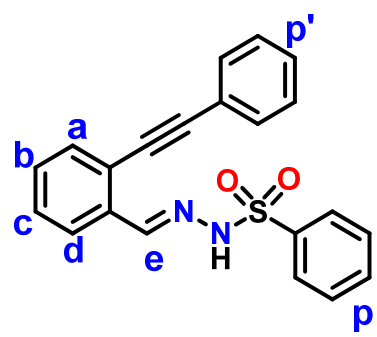

$\mathbf{E}$

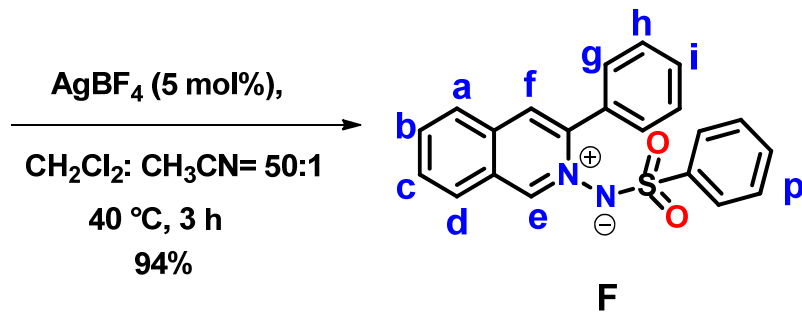

$\mathbf{F}$

Compound $\mathbf{E}$ (50 mg, $138 \mu \mathrm{mol})$ was dissolved in $5 \mathrm{~mL}$ of dichloromethane. Then $\mathrm{AgBF}_{4}(1.35$ $\mathrm{mg}, 6.93 \mu \mathrm{mol}, 5 \mathrm{~mol} \%$ ) in $100 \mu \mathrm{L}$ of acetonitrile was added and the mixture was heated for $3 \mathrm{~h}$ at $40{ }^{\circ} \mathrm{C}$. The solvent was removed and the residue was purified by chromatography (silica gel, $\left.\mathrm{MeOH} / \mathrm{DCM}=1: 8, R_{f}=0.3\right)$ to afford product $\mathbf{F}(47 \mathrm{mg}, 130 \mu \mathrm{mol}, 94 \%)$ as a white solid. IR (KBr): $\tilde{v}=521,560,621,642,723,1027,1051,1092,1121,1132,1176,1191,1221,1233$, $1269,1292,1323,1344,1356,1379,1393,1452,1470,1506,1572,1599,2738,2858,2829$, 2917, $2980 \mathrm{~cm}^{-1} .{ }^{1} \mathbf{H}$ NMR (400 MHz, $\left.\mathbf{C D C l}_{3}\right): \delta=7.17-7.33$ (m, 8H, h-, i-, p-H), 7.35-7.44 (m, 2H, g-H), 7.84-7.89 (m, 2H, f-, a-H), 7.95-8.05 (m, 1H, c-, b-H), 9.57 (s, 1H, e-H) ppm. 


\section{Catalytic experiment A1}

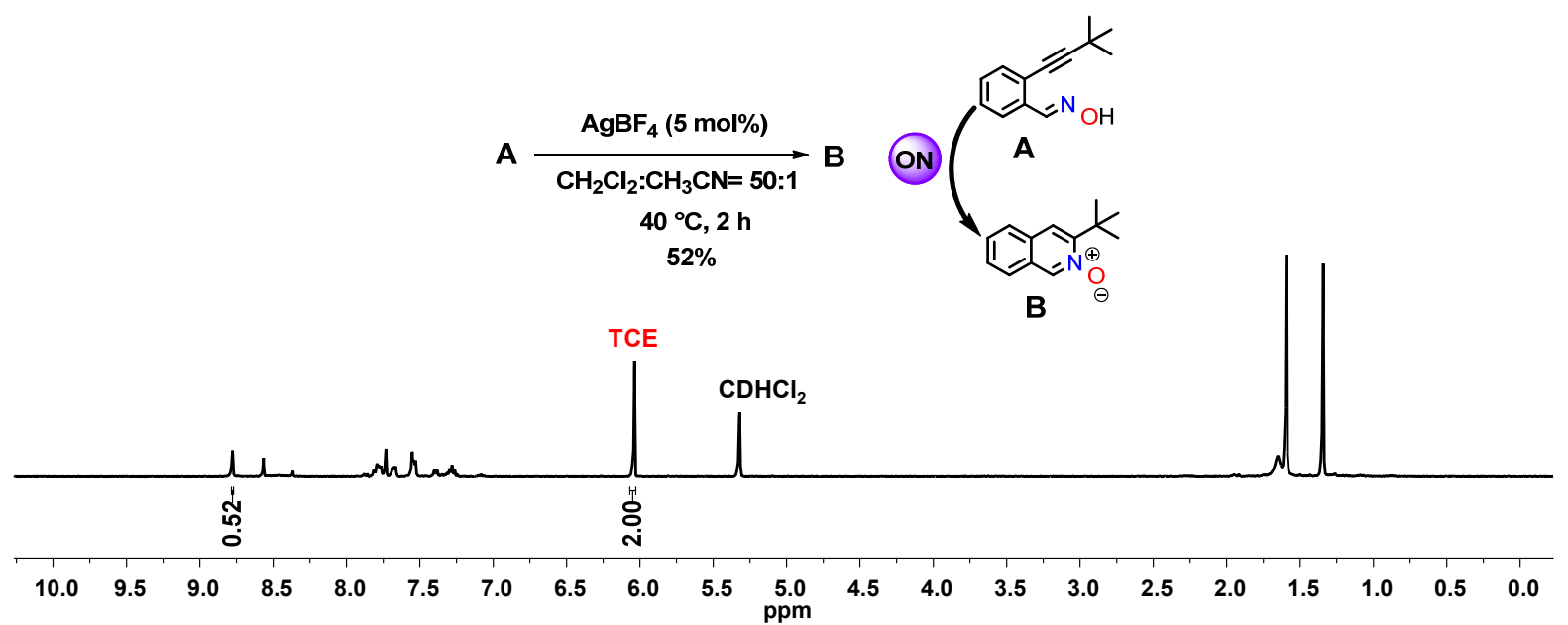

Figure S32. ${ }^{1} \mathrm{H}$ NMR (400 MHz, $\left.298 \mathrm{~K}\right)$ spectrum obtained after heating the 20:1:20 reaction mixture of $\mathbf{A}$ (21.6 $\mathrm{mM}), \mathrm{AgBF}_{4}(1.08 \mathrm{mM}, 5 \mathrm{~mol} \%)$ and 1,1,2,2-tetrachloroethane (TCE) in $500 \mu \mathrm{L}$ of $\mathrm{CD}_{2} \mathrm{Cl}_{2} / \mathrm{CD}_{3} \mathrm{CN}=50: 1$ at $40{ }^{\circ} \mathrm{C}$ for $2 \mathrm{~h}$. The integration demonstrated that $\mathbf{B}$ was formed in $52 \%$ yield.

\section{Catalytic experiment A2}

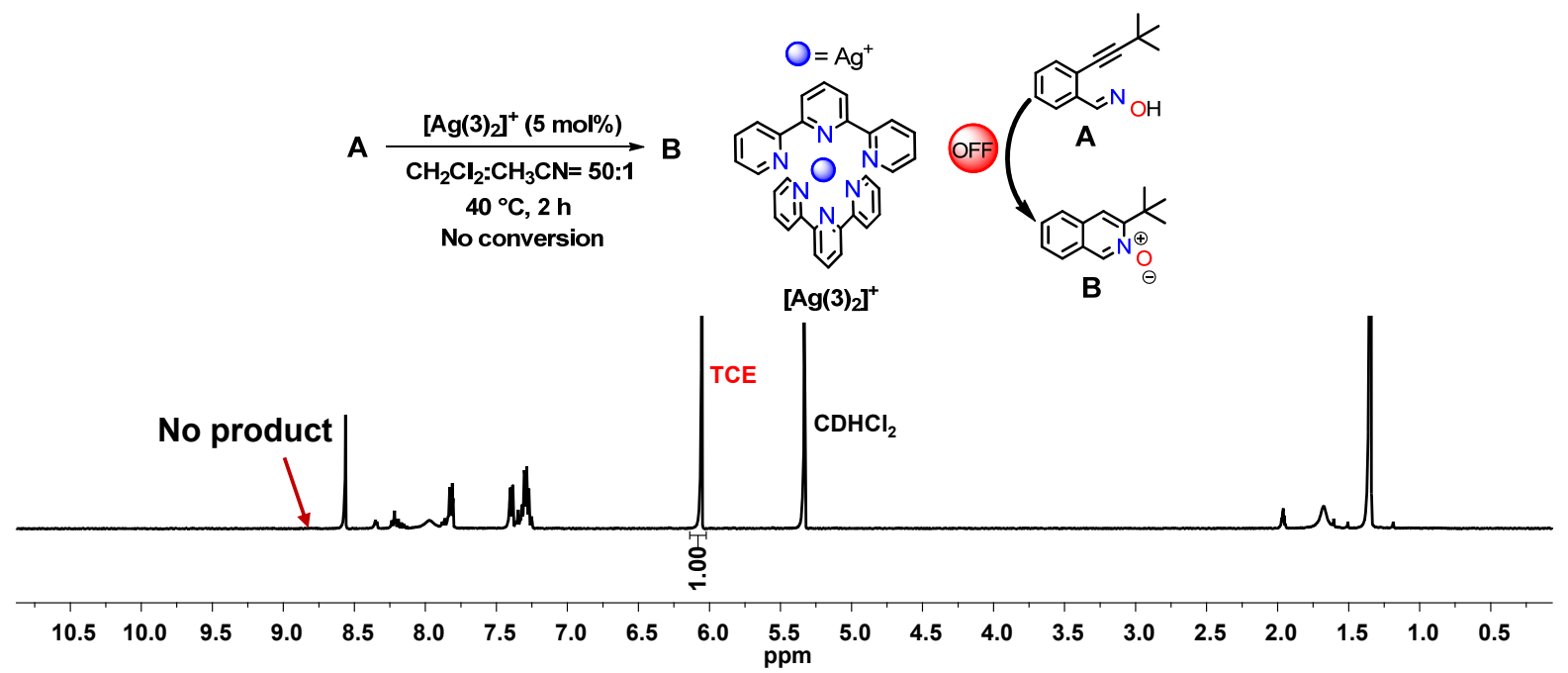

Figure S33. ${ }^{1} \mathrm{H}$ NMR (400 MHz, $\left.\mathrm{CD}_{2} \mathrm{Cl}_{2}: \mathrm{CD}_{3} \mathrm{CN}=50: 1,298 \mathrm{~K}\right)$ spectrum obtained after heating the 20:1:20 reaction mixture of $\mathbf{A}(21.6 \mathrm{mM}),\left[\mathrm{Ag}(3)_{2}\right]^{+}(1.08 \mathrm{mM}, 5 \mathrm{~mol} \%)$ and 1,1,2,2-tetrachloroethane (TCE) in $500 \mu \mathrm{L}$ of $\mathrm{CD}_{2} \mathrm{Cl}_{2} / \mathrm{CD}_{3} \mathrm{CN}=50: 1$ at $40{ }^{\circ} \mathrm{C}$ for $2 \mathrm{~h}$. It didn't show any conversion to product $\mathbf{B}$. 


\section{Catalytic experiment A3}
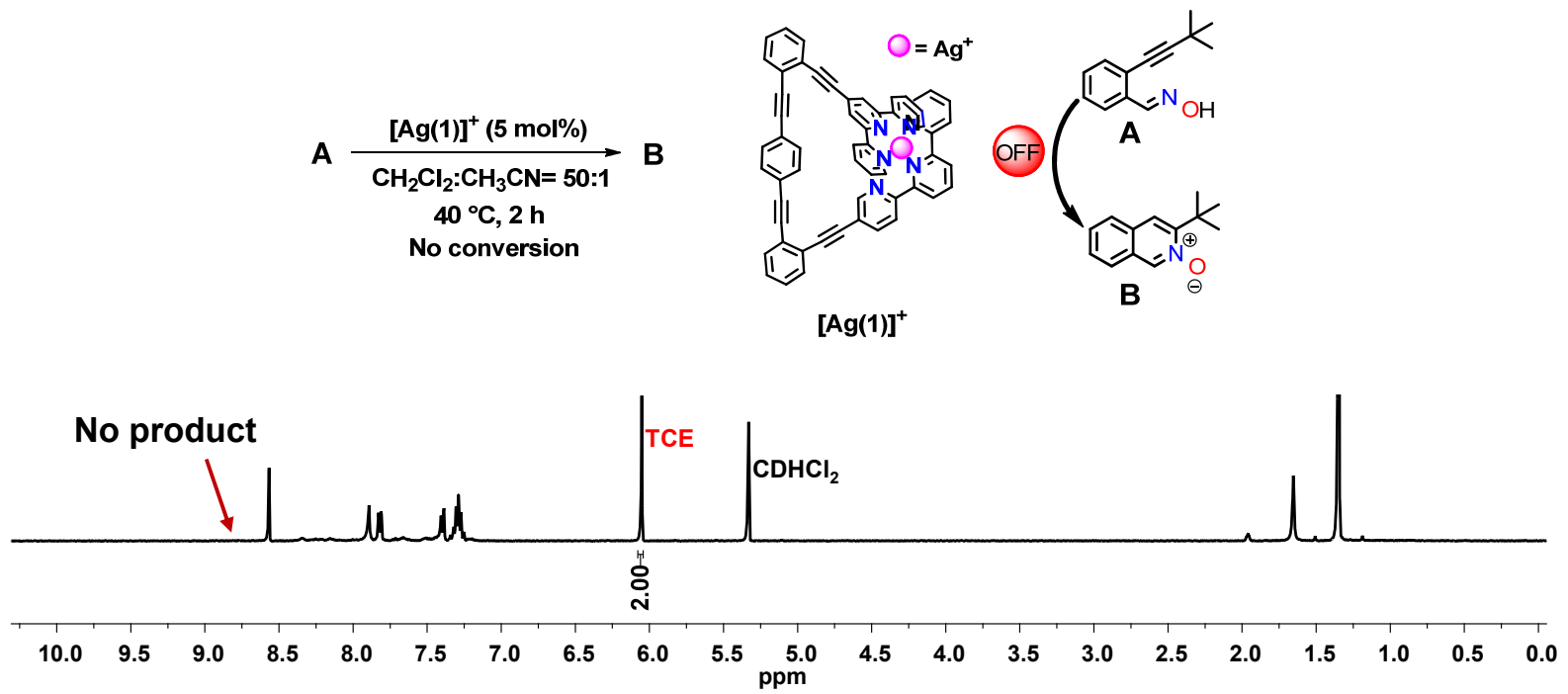

Figure S34. ${ }^{1} \mathrm{H}$ NMR (400 MHz, $\left.298 \mathrm{~K}\right)$ spectrum obtained after heating the 20:1:20 reaction mixture of $\mathbf{A}$ (21.6 $\mathrm{mM}),[\mathrm{Ag}(\mathbf{1})]^{+}(1.08 \mathrm{mM}, 5 \mathrm{~mol} \%)$ and 1,1,2,2-tetrachloroethane (TCE) in $500 \mu \mathrm{L}$ of $\mathrm{CD}_{2} \mathrm{Cl}_{2} / \mathrm{CD}_{3} \mathrm{CN}=50: 1$ at 40 ${ }^{\circ} \mathrm{C}$ for 2. It didn't show any conversion to product $\mathbf{B}$.

\section{Catalytic experiment A4}

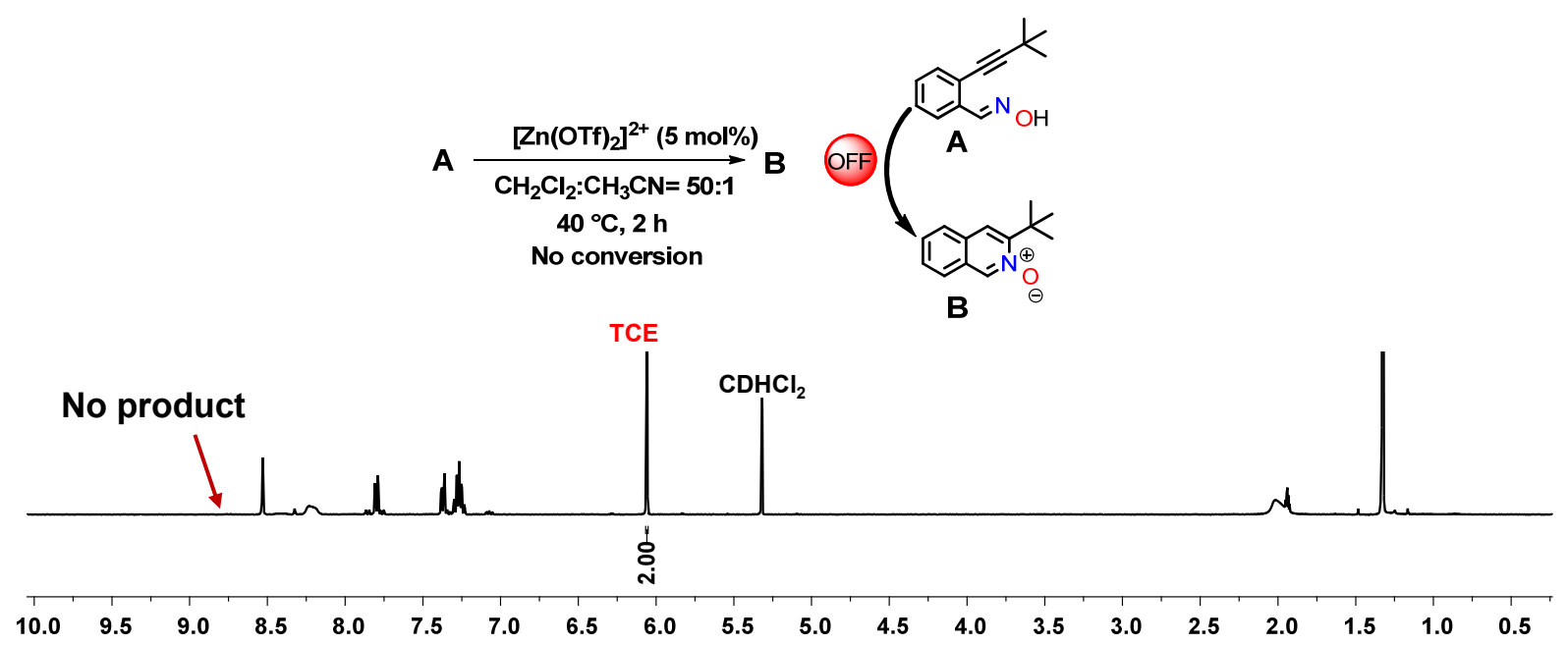

Figure S35. ${ }^{1} \mathrm{H}$ NMR ( $400 \mathrm{MHz}, \mathrm{CD}_{2} \mathrm{Cl}_{2}: \mathrm{CD}_{3} \mathrm{CN}=50: 1,298 \mathrm{~K}$ ) spectrum obtained after heating the 20:1:20 reaction mixture of $\mathbf{A}(21.6 \mathrm{mM}), \mathrm{Zn}(\mathrm{OTf})_{2}(1.08 \mathrm{mM}, 5 \mathrm{~mol} \%)$ and 1,1,2,2-tetrachloroethane (TCE) in $500 \mu \mathrm{L}$ of $\mathrm{CD}_{2} \mathrm{Cl}_{2} / \mathrm{CD}_{3} \mathrm{CN}=50: 1$ at $40{ }^{\circ} \mathrm{C}$ for $2 \mathrm{~h}$. It didn't show any conversion to product $\mathbf{B}$. 


\section{Catalytic experiment A5}

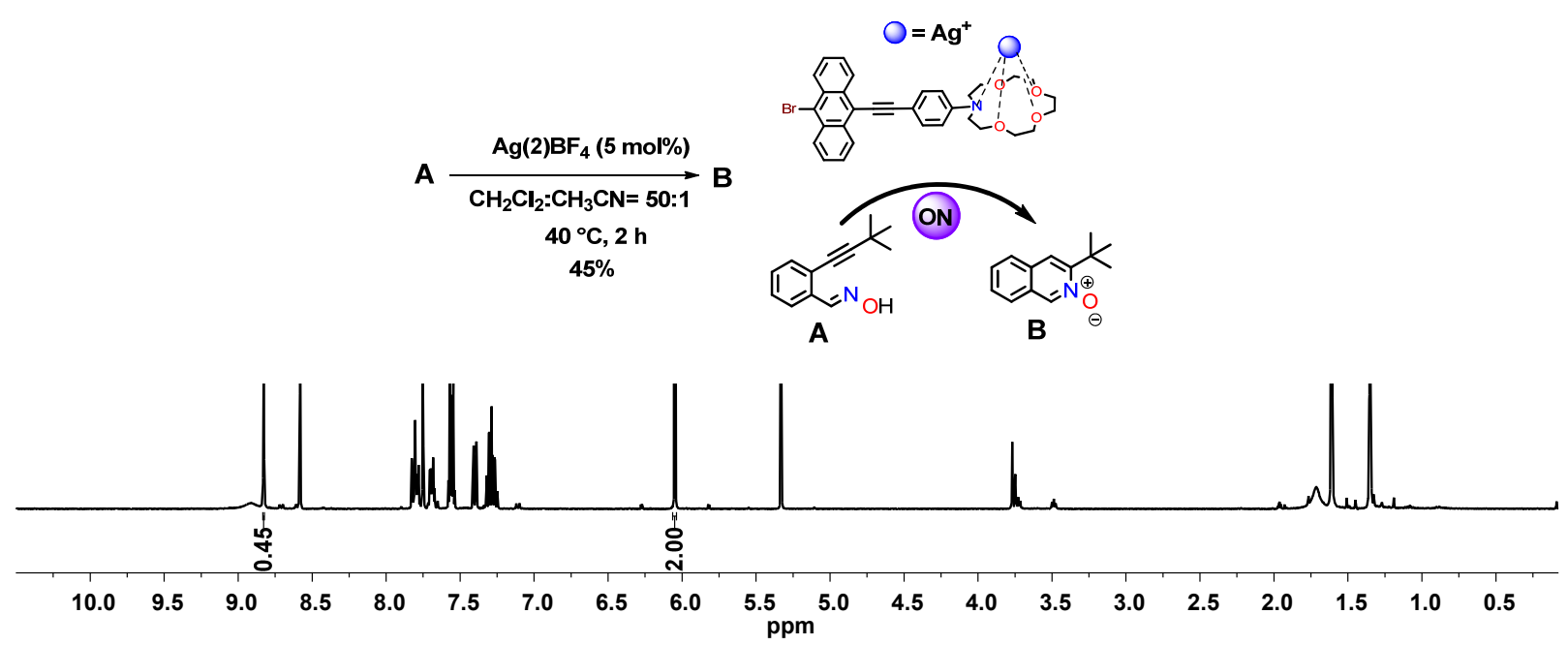

Figure S36. ${ }^{1} \mathrm{H}$ NMR (400 MHz, $\left.\mathrm{CD}_{2} \mathrm{Cl}_{2}: \mathrm{CD}_{3} \mathrm{CN}=50: 1,298 \mathrm{~K}\right)$ spectrum obtained after heating the 20:1:20 reaction mixture of $\mathbf{A}(21.6 \mathrm{mM}),[\mathrm{Ag}(2)] \mathrm{BF}_{4}(1.08 \mathrm{mM}, 5 \mathrm{~mol} \%)$ and 1,1,2,2-tetrachloroethane (TCE) in $500 \mu \mathrm{L}$ of $\mathrm{CD}_{2} \mathrm{Cl}_{2} / \mathrm{CD}_{3} \mathrm{CN}=50: 1$ at $40{ }^{\circ} \mathrm{C}$ for $2 \mathrm{~h}$. The integration demonstrated that $\mathbf{B}$ was formed in $45 \%$ yield. 


\section{Catalytic experiment A6}

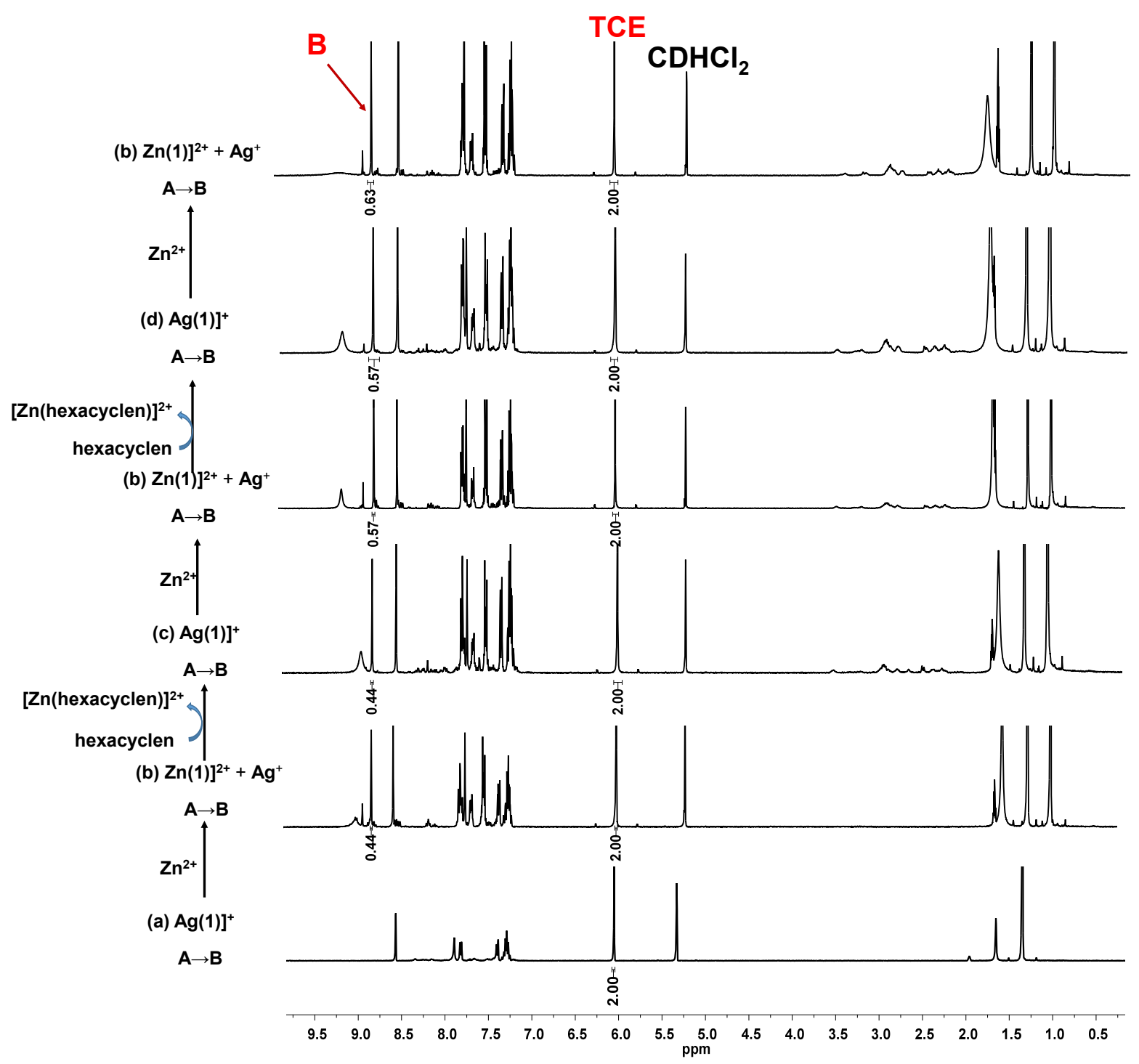

Figure S37. Catalytic cycles. ${ }^{1} \mathrm{H}$ NMR (400 MHz, $\left.\mathrm{CD}_{2} \mathrm{Cl}_{2}: \mathrm{CD}_{3} \mathrm{CN}=50: 1,298 \mathrm{~K}\right)$ spectrum obtained after (a) heating the 1:1:20 reaction mixture of $\mathbf{1}(1.08 \mathrm{mM}, 5 \mathrm{~mol} \%), \mathrm{AgBF}_{4}(1.08 \mathrm{mM}, 5 \mathrm{~mol} \%), \mathbf{A}(21.6 \mathrm{mM}, 100 \mathrm{~mol} \%)$ in $500 \mu \mathrm{L}$ of $\mathrm{CD}_{2} \mathrm{Cl}_{2} / \mathrm{CD}_{3} \mathrm{CN}=50: 1$ at $40{ }^{\circ} \mathrm{C}$ for $2 \mathrm{~h}$ in an NMR tube revealed that formation of cyclized product $\mathbf{B}$ (singlet at $\delta 8.82 \mathrm{ppm}$ ) was OFF. (b) Addition of 1.0 equiv. of $\mathrm{Zn}(\mathrm{OTf})_{2}$ with respect to 1 and heating at $40{ }^{\circ} \mathrm{C}$ for 2 $\mathrm{h}$ resulted in formation of $44 \%$ of cyclized product $\mathbf{B}$ (calculated with respect to internal standard TCE). (c) After addition of 1.0 equiv. of hexacyclen with respect to $\mathbf{1}$ and adding substrate $\mathbf{A}$ (to regenerate starting conc.), the solution was heated at $40{ }^{\circ} \mathrm{C}$ for $2 \mathrm{~h}$. Formation of cyclized product B was OFF. (d) Addition of 1.0 equiv. of $\mathrm{Zn}(\mathrm{OTf})_{2}$ with respect to 1 and heating at $40{ }^{\circ} \mathrm{C}$ for $2 \mathrm{~h}$ resulted in an increase of cyclized product $\mathbf{B}$ by $13 \%$ (total yield 57\%). (e) After addition of 1.0 equiv. of hexacyclen with respect to $\mathbf{1}$ and adding substrate $\mathbf{A}$ (to regenerate starting conc.), the resulting solution was heated at $40{ }^{\circ} \mathrm{C}$ for $2 \mathrm{~h}$. Formation of the cyclized product B was OFF 
(overall yield 57\%). (f) Addition of 1.0 equiv. of $\mathrm{Zn}(\mathrm{OTf})_{2}$ with respect to 1 and heating at $40{ }^{\circ} \mathrm{C}$ for $2 \mathrm{~h}$ resulted in an increase of cyclized product $\mathbf{B}$ by $6 \%$ (total yield $63 \%$ ).

\section{Catalytic experiment A7}

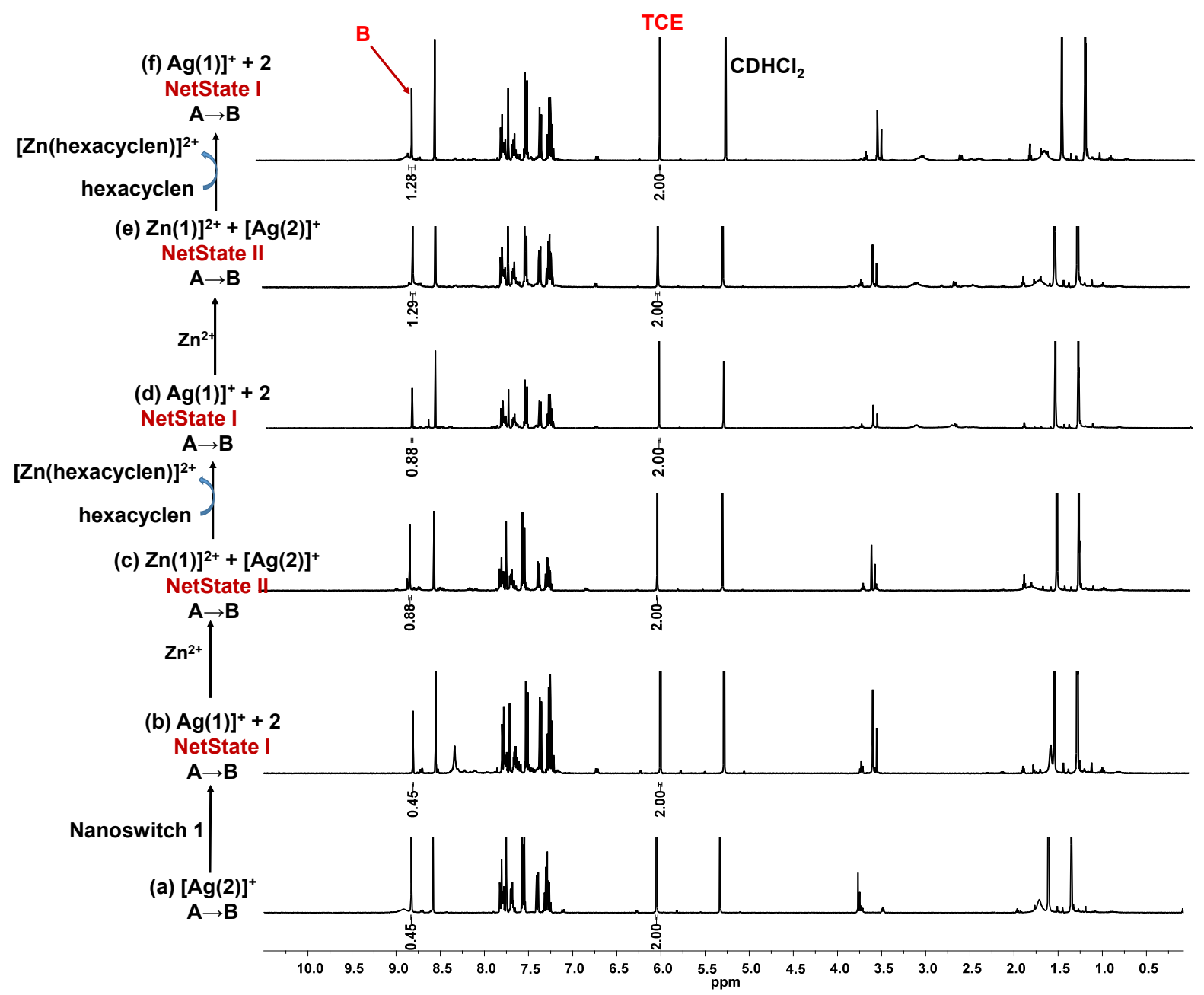

Figure S38. Catalytic cycles. ${ }^{1} \mathrm{H}$ NMR $\left(400 \mathrm{MHz}, \mathrm{CD}_{2} \mathrm{Cl}_{2}: \mathrm{CD}_{3} \mathrm{CN}=50: 1,298 \mathrm{~K}\right)$ spectrum obtained after (a) heating the 1:1:20 reaction mixture of 2 (1.08 mM, $5 \mathrm{~mol} \%), \mathrm{AgBF}_{4}(1.08 \mathrm{mM}, 5 \mathrm{~mol} \%), \mathbf{A}(21.6 \mathrm{mM}, 100 \mathrm{~mol} \%)$ in $500 \mu \mathrm{L}$ of $\mathrm{CD}_{2} \mathrm{Cl}_{2} / \mathrm{CD}_{3} \mathrm{CN}=50: 1$ at $40{ }^{\circ} \mathrm{C}$ for $2 \mathrm{~h}$ in an NMR tube revealed that cyclized product $\mathbf{B}$ (singlet at $\delta$ $8.82 \mathrm{ppm}$ ) was formed (yield $=45 \%$ calculated with respect to internal standard TCE). (b) After addition of 1.0 equiv. of nanoswitch $\mathbf{1}$ with respect to ligand $\mathbf{2}$ and adding substrate $\mathbf{A}$ (to regenerate starting conc.), followed by subsequent heating at $40{ }^{\circ} \mathrm{C}$ for $2 \mathrm{~h}$. Formation of cyclized product $\mathbf{B}$ was OFF. (c) Addition of 1.0 equiv. of $\mathrm{Zn}(\mathrm{OTf})_{2}$ with respect to 1 and heating at $40{ }^{\circ} \mathrm{C}$ for $2 \mathrm{~h}$ resulted in an increase of cyclized product $\mathbf{B}$ by $43 \%$ (total yield 88\%). (d) After addition of 1.0 equiv. of hexacyclen with respect to $\mathbf{1}$ and adding substrate $\mathbf{A}$ (to regenerate starting conc.), the solution was heated at $40{ }^{\circ} \mathrm{C}$ for $2 \mathrm{~h}$. Formation of cyclized product $\mathbf{B}$ was OFF. (e) Addition of 1.0 equiv. of $\mathrm{Zn}(\mathrm{OTf})_{2}$ with respect to 1 and heating at $40^{\circ} \mathrm{C}$ for $2 \mathrm{~h}$ resulted in an increase of cyclized product $\mathbf{B}$ by 
$41 \%$ (total yield 129\%). (f) After addition of 1.0 equiv. of hexacyclen with respect to $\mathbf{1}$ and adding substrate $\mathbf{A}$ (to regenerate starting conc.) the resulting solution was heated at $40{ }^{\circ} \mathrm{C}$ for $2 \mathrm{~h}$. Formation of cyclized product $\mathbf{B}$ was OFF (overall yield 129\%).

\section{Catalytic experiment A8}

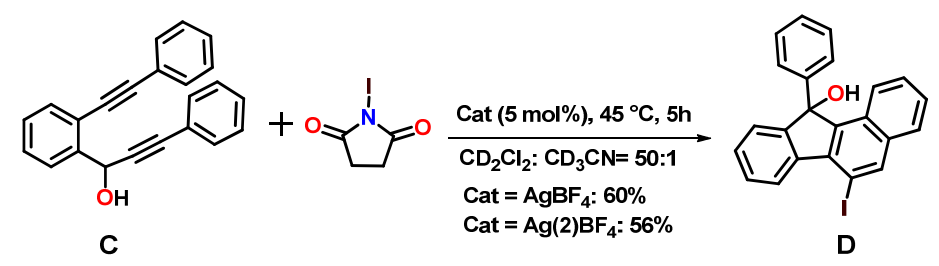

(b) $\mathrm{Cat}=\mathrm{Ag}(2) \mathrm{BF}_{4}$

$\mathrm{C} \rightarrow \mathrm{D}$
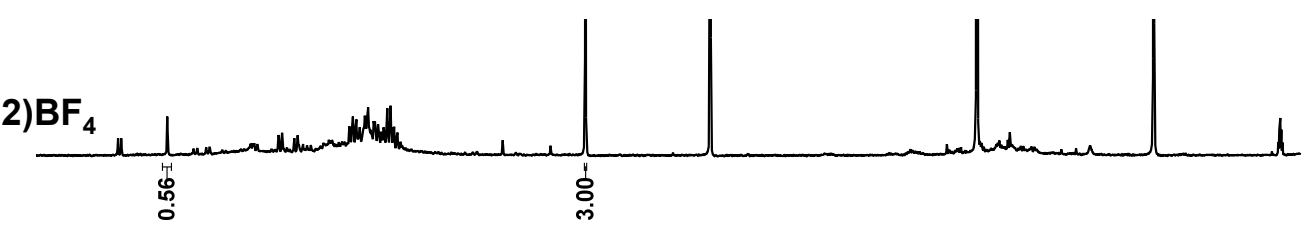

(a) $\mathrm{Cat}=\mathrm{AgBF}_{4}$

$$
C \rightarrow D
$$

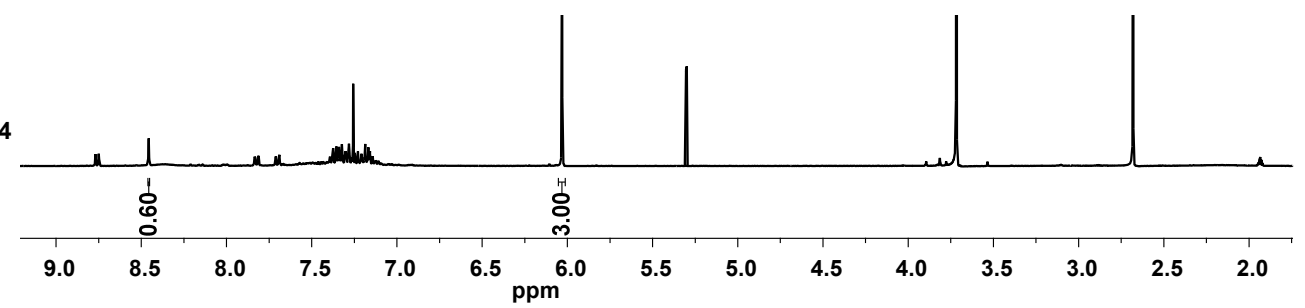

Figure S39. ${ }^{1} \mathrm{H} \mathrm{NMR}\left(400 \mathrm{MHz}, \mathrm{CD}_{2} \mathrm{Cl}_{2}: \mathrm{CD}_{3} \mathrm{CN}=50: 1,298 \mathrm{~K}\right.$ ) spectrum obtained after (a) heating the 20:20:1:20 reaction mixture of $\mathbf{C}(21.6 \mathrm{mM}), N$-iodosuccinimide $(25.9 \mathrm{mM}), \mathrm{AgBF}_{4}(1.08 \mathrm{mM}, 5 \mathrm{~mol} \%)$ and 1,3,5trimethoxybenzene (standard) in $500 \mu \mathrm{L}$ of $\mathrm{CD}_{2} \mathrm{Cl}_{2} / \mathrm{CD}_{3} \mathrm{CN}=50: 1$ at $40{ }^{\circ} \mathrm{C}$ for $2 \mathrm{~h}$. The integration demonstrated that $\mathbf{D}$ was formed in 60\% yield. (b) Heating the 20:20:1:1:20 reaction mixture of $\mathbf{C}(21.6 \mathrm{mM}), N$-iodosuccinimide (25.9 mM), $\mathrm{AgBF}_{4}(1.08 \mathrm{mM}, 5 \mathrm{~mol} \%)$, ligand 2 (1.08 mM, $\left.5 \mathrm{~mol} \%\right)$ and 1,3,5-trimethoxybenzene (standard) in 500 $\mu \mathrm{L}$ of $\mathrm{CD}_{2} \mathrm{Cl}_{2} / \mathrm{CD}_{3} \mathrm{CN}=50: 1$ at $40{ }^{\circ} \mathrm{C}$ for $2 \mathrm{~h}$. The integration demonstrated that $\mathbf{D}$ was formed in $56 \%$ yield. 


\section{Catalytic experiment A9}

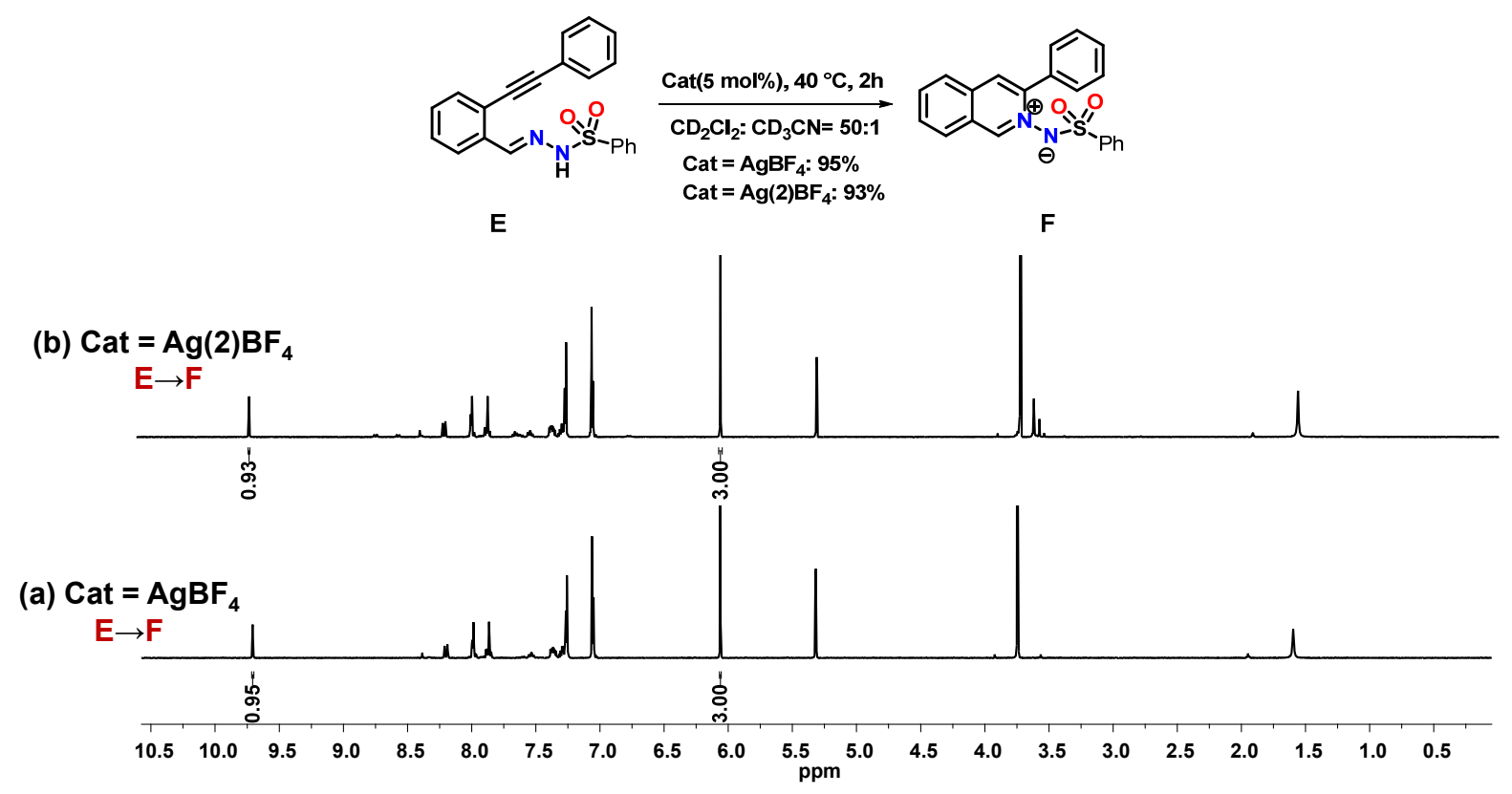

Figure S40. ${ }^{1} \mathrm{H}$ NMR (400 MHz, $\mathrm{CD}_{2} \mathrm{Cl}_{2}: \mathrm{CD}_{3} \mathrm{CN}=50: 1,298 \mathrm{~K}$ ) spectrum obtained after (a) heating the 20:1:20 reaction mixture of $\mathbf{E}(21.6 \mathrm{mM}), \mathrm{AgBF}_{4}(1.08 \mathrm{mM}, 5 \mathrm{~mol} \%)$ and 1,3,5-trimethoxybenzene (standard) in $500 \mu \mathrm{L}$ of $\mathrm{CD}_{2} \mathrm{Cl}_{2} / \mathrm{CD}_{3} \mathrm{CN}=50: 1$ at $40{ }^{\circ} \mathrm{C}$ for $2 \mathrm{~h}$. The integration demonstrated that $\mathbf{F}$ was formed in $95 \%$ yield. (b) Heating the 20:20:1:1:20 reaction mixture of $\mathbf{E}(21.6 \mathrm{mM}), \mathrm{AgBF}_{4}(1.08 \mathrm{mM}, 5 \mathrm{~mol} \%)$, ligand 2 and 1,3,5trimethoxybenzene (standard) in $500 \mu \mathrm{L}$ of $\mathrm{CD}_{2} \mathrm{Cl}_{2} / \mathrm{CD}_{3} \mathrm{CN}=50: 1$ at $40{ }^{\circ} \mathrm{C}$ for $2 \mathrm{~h}$. The integration demonstrated that $\mathbf{F}$ was formed in $93 \%$ yield.

\section{Catalytic experiment A10}

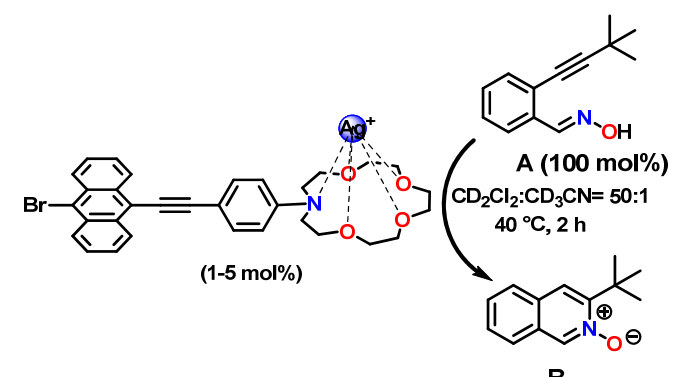

B

Substrate A (21.6 mM) and separately prepared $[\operatorname{Ag}(2)]^{+}(1-5 \mathrm{~mol} \%)$ was dissolved in $500 \mu \mathrm{L}$ $\mathrm{CD}_{2} \mathrm{Cl}_{2}$ and $\mathrm{CD}_{3} \mathrm{CN}$ (50:1) and the reaction was heated at $40{ }^{\circ} \mathrm{C}$ for $2 \mathrm{~h}$, then the mixture was cooled to room temperature and directly subjected to ${ }^{1} \mathrm{H}$ NMR measurements. The yield of product $\mathbf{B}$ depending on the amount of catalyst is shown in Figure S41. 
(e) $5 \mathrm{~mol} \%$

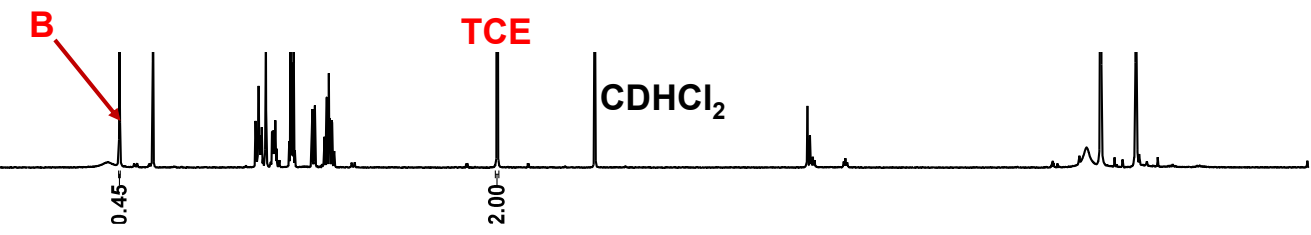

(d) $4 \mathrm{~mol} \%$

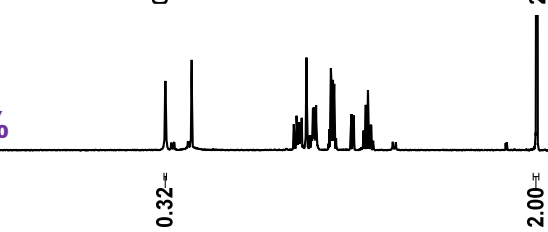

(c) $3 \mathrm{~mol} \%$
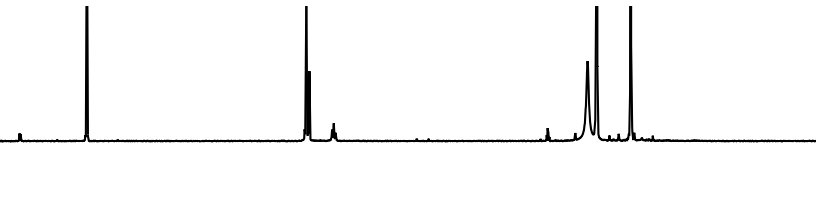

(b) $2 \mathrm{~mol} \%$

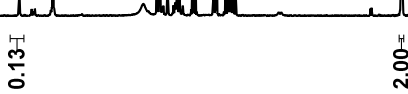

(a) $1 \mathrm{~mol} \%$

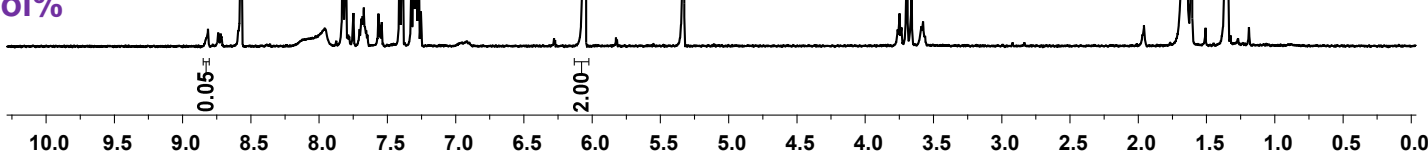

Figure S41. ${ }^{1} \mathrm{H}$ NMR spectra ( $400 \mathrm{MHz}, \mathrm{CD}_{2} \mathrm{Cl}_{2}: \mathrm{CD}_{3} \mathrm{CN}=50: 1,298 \mathrm{~K}$ ) of the reaction of substrate $\mathbf{A}$ in presence of $[\operatorname{Ag}(2)]^{+}(1-5 \mathrm{~mol} \%)$ at $40{ }^{\circ} \mathrm{C}$ for $4 \mathrm{~h}$. Product $\mathbf{B}$ shows up at $8.82 \mathrm{ppm}$ in the spectrum (integration given). 


\section{ESI-MS Spectra}

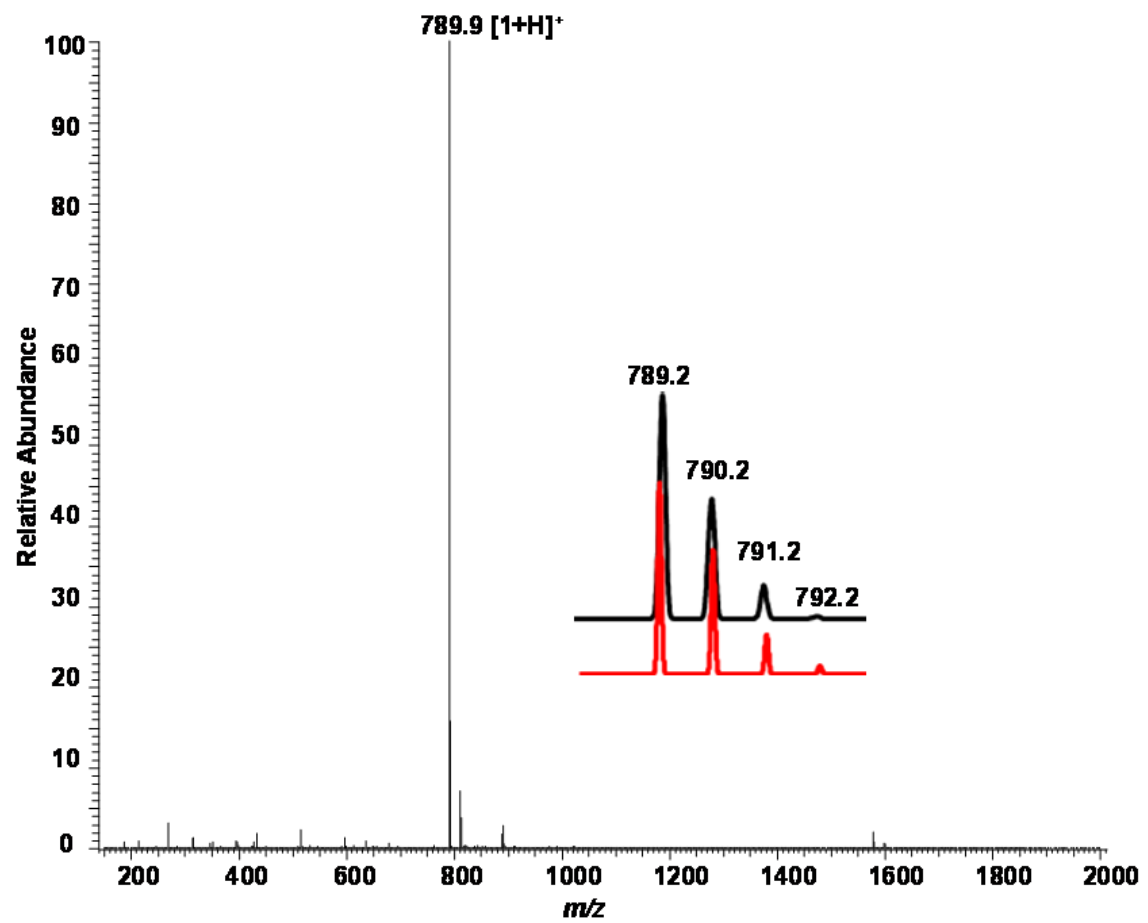

Figure S42. ESI-MS of 1 after protonation.

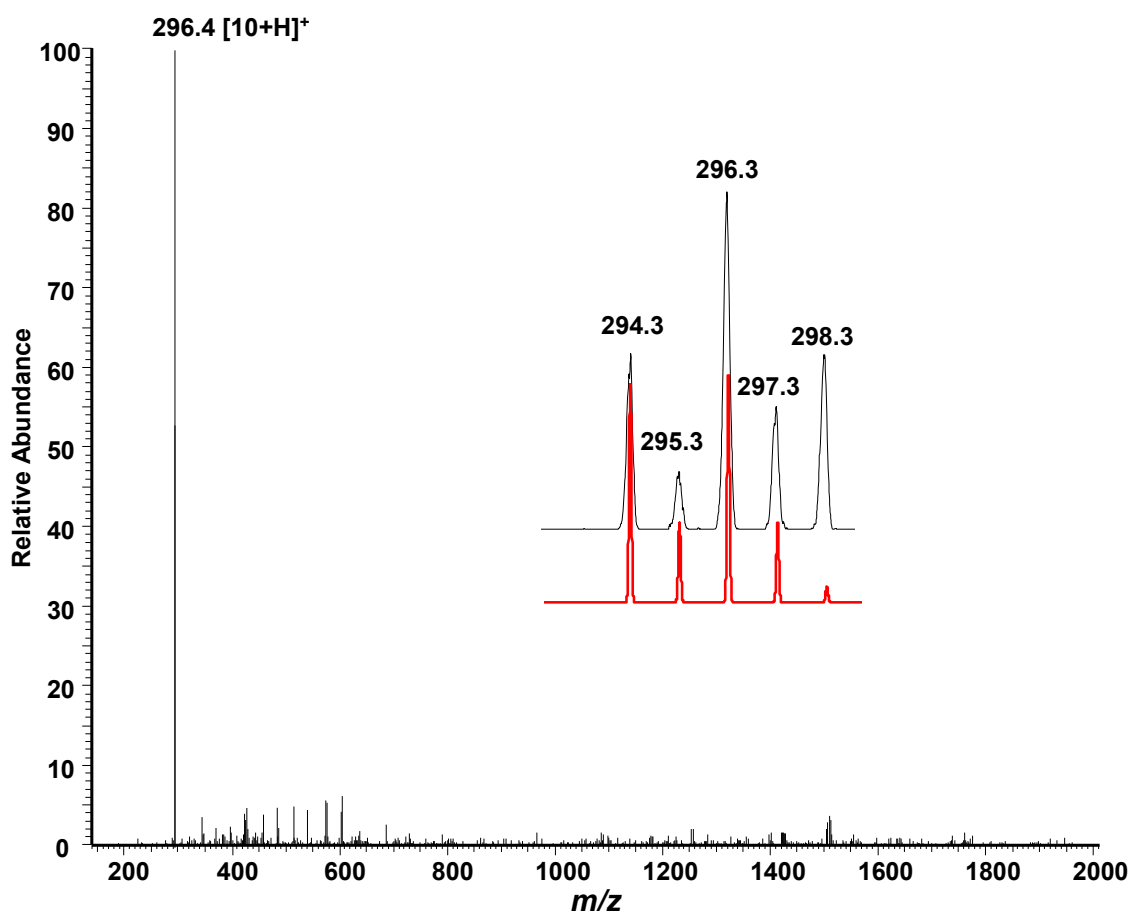

Figure S43. ESI-MS of $\mathbf{1 0}$ after protonation. 


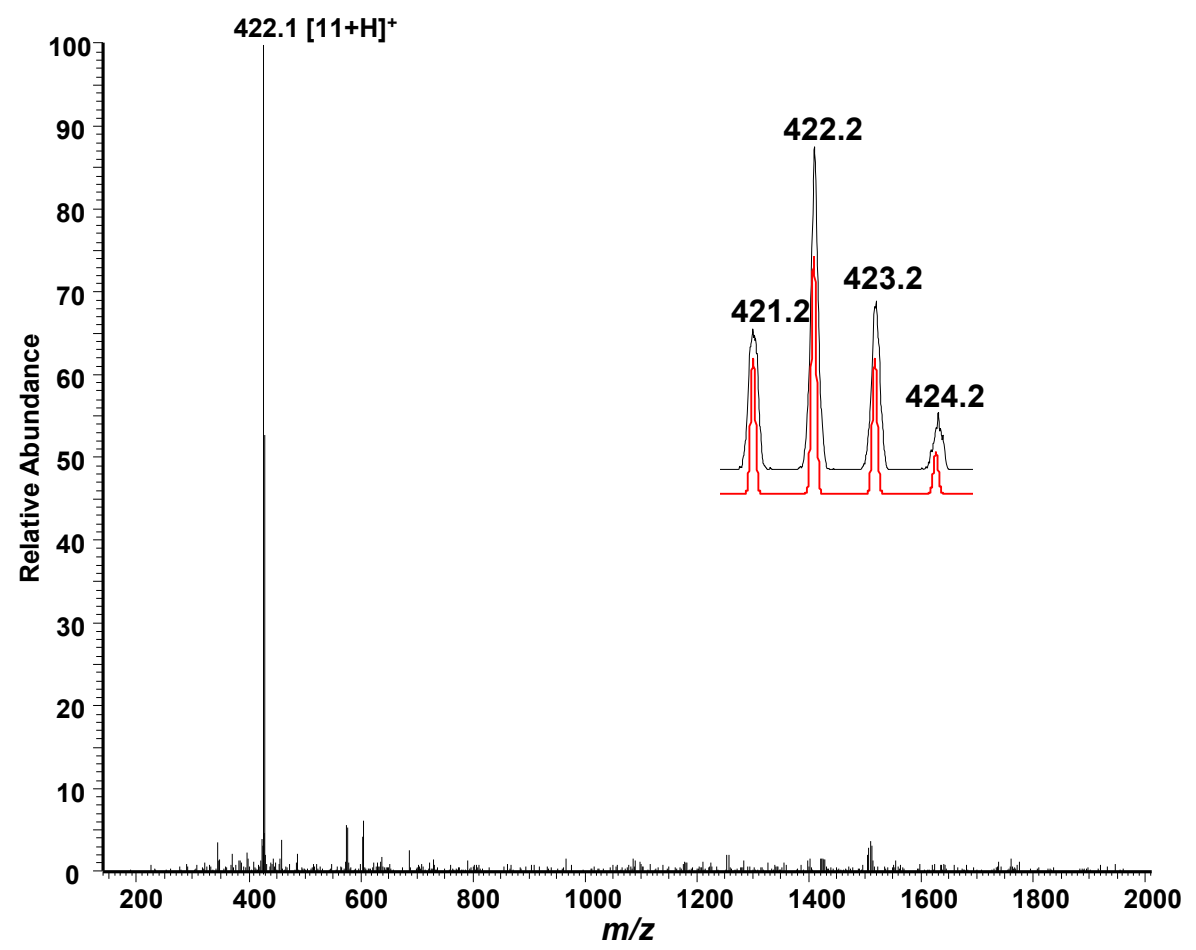

Figure S44. ESI-MS of 11 after protonation.

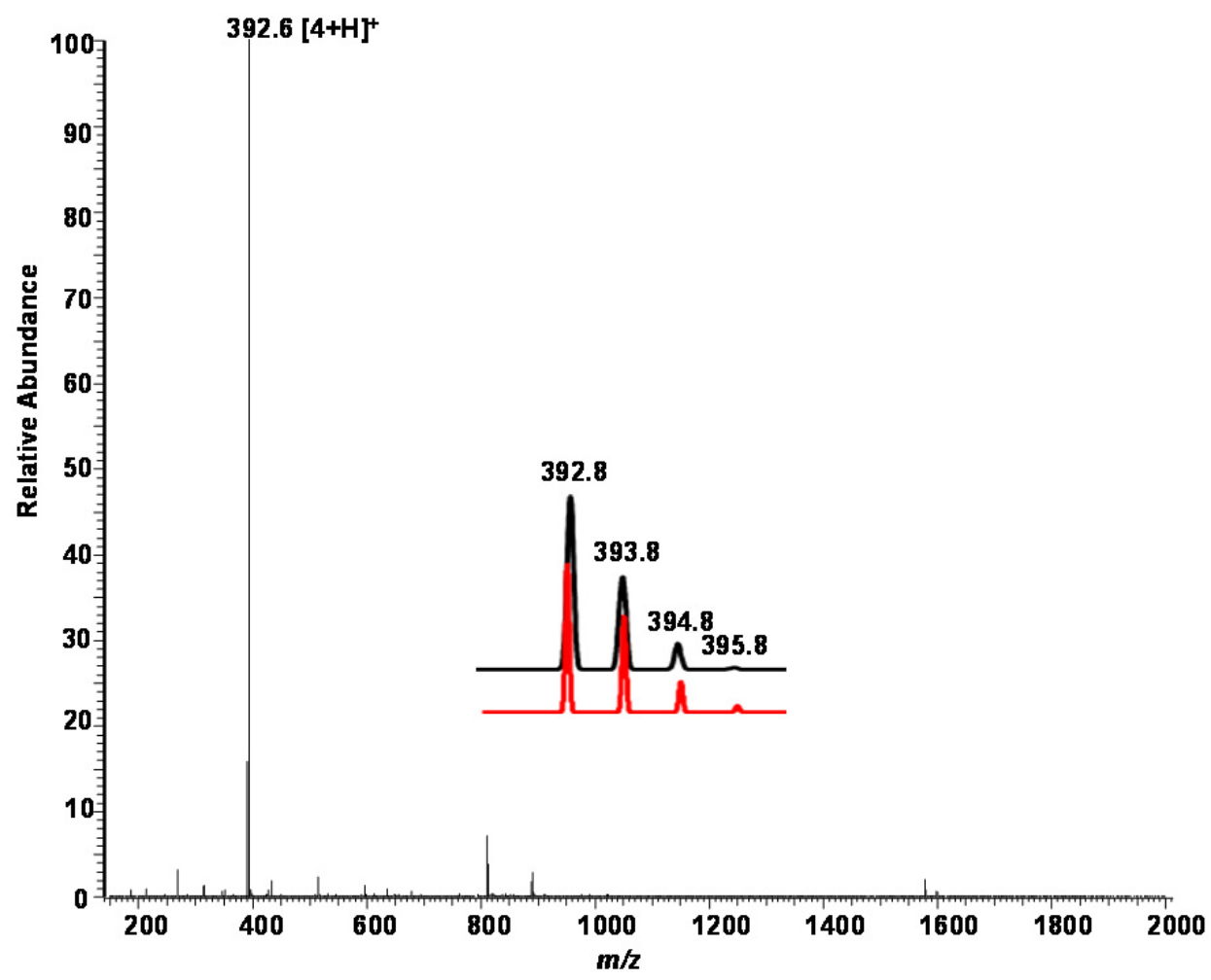

Figure S45. ESI-MS of $\mathbf{4}$ after protonation. 


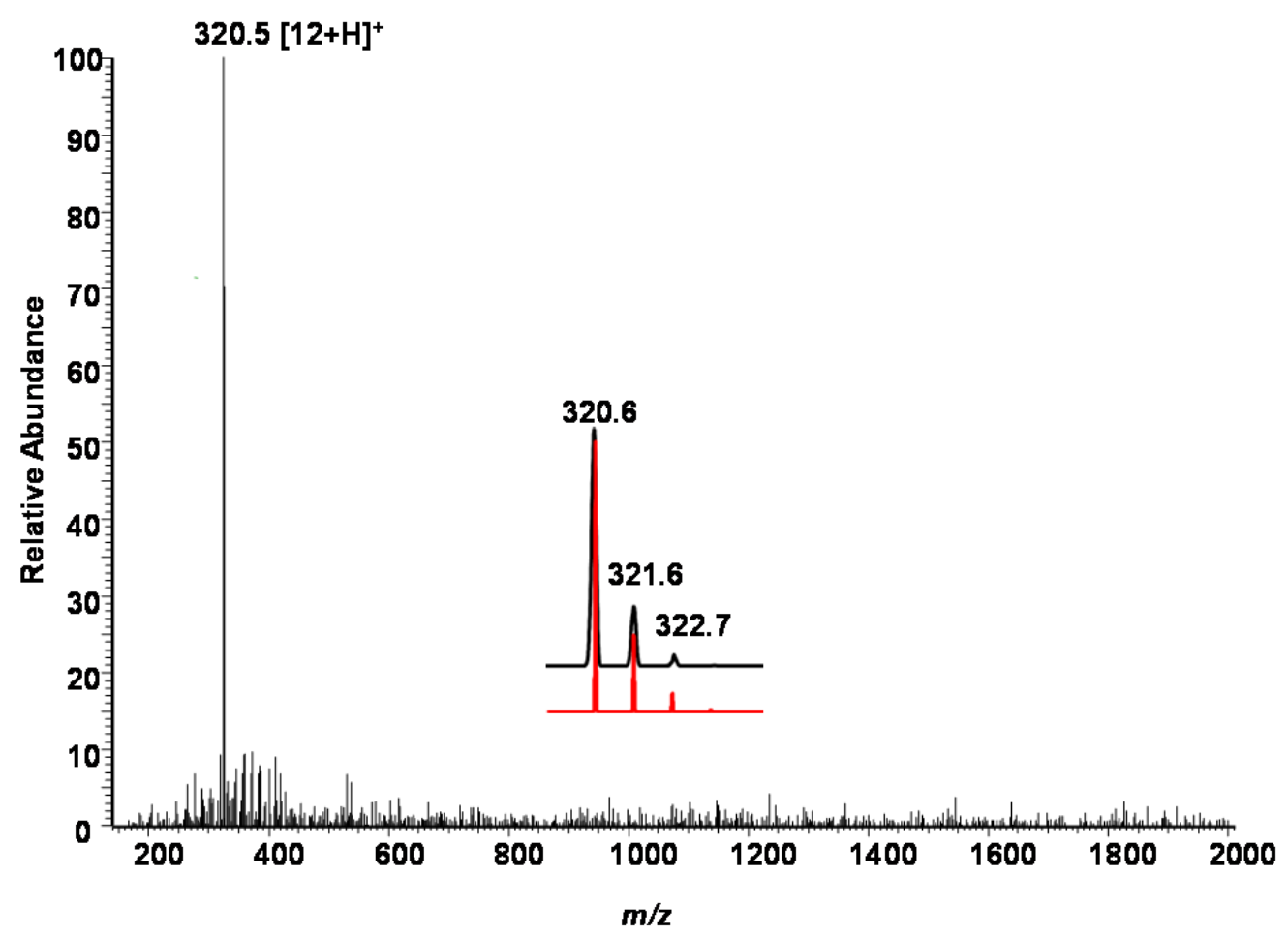

Figure S46. ESI-MS of 12 after protonation.

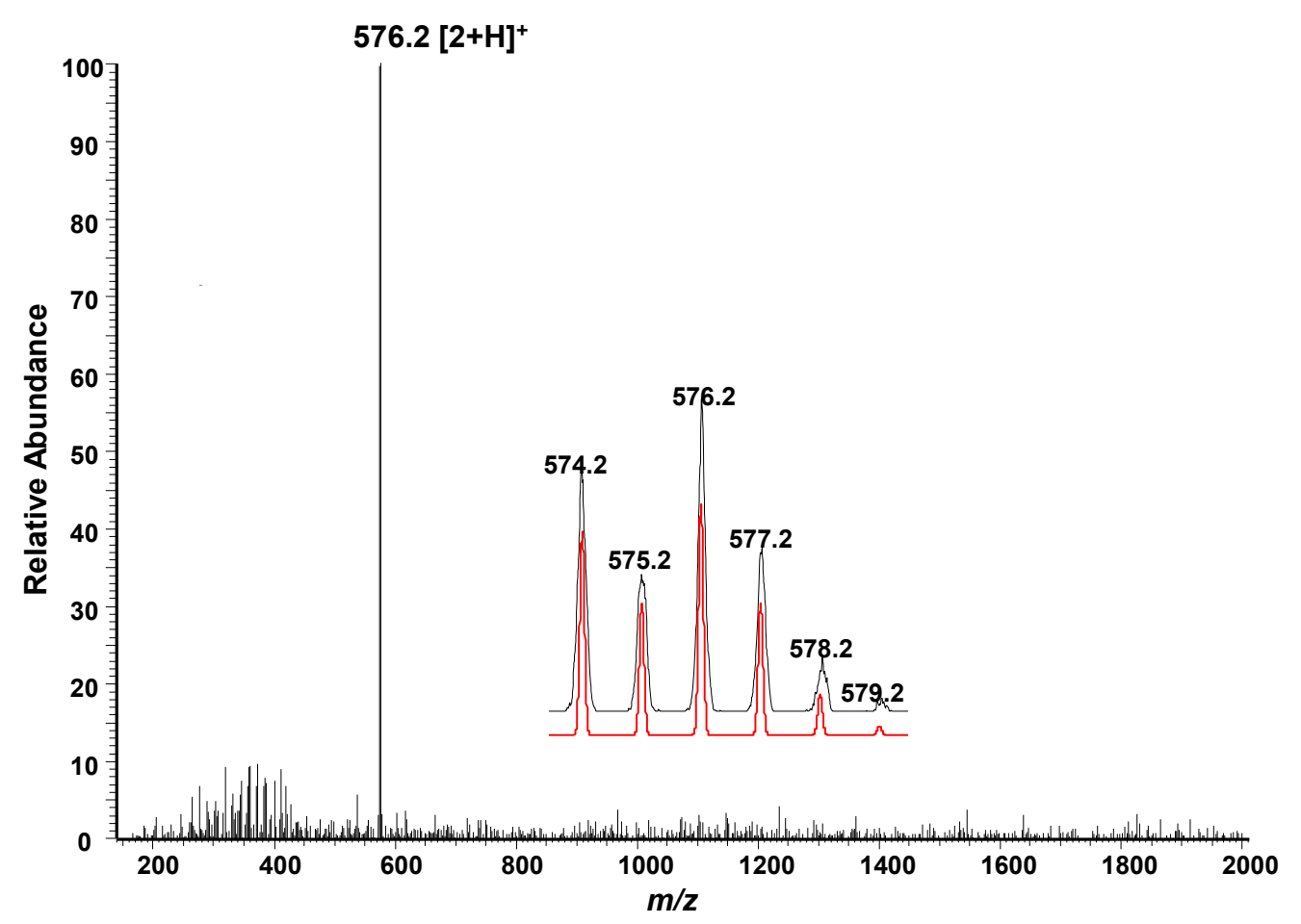

Figure S47. ESI-MS of 2 after protonation. 


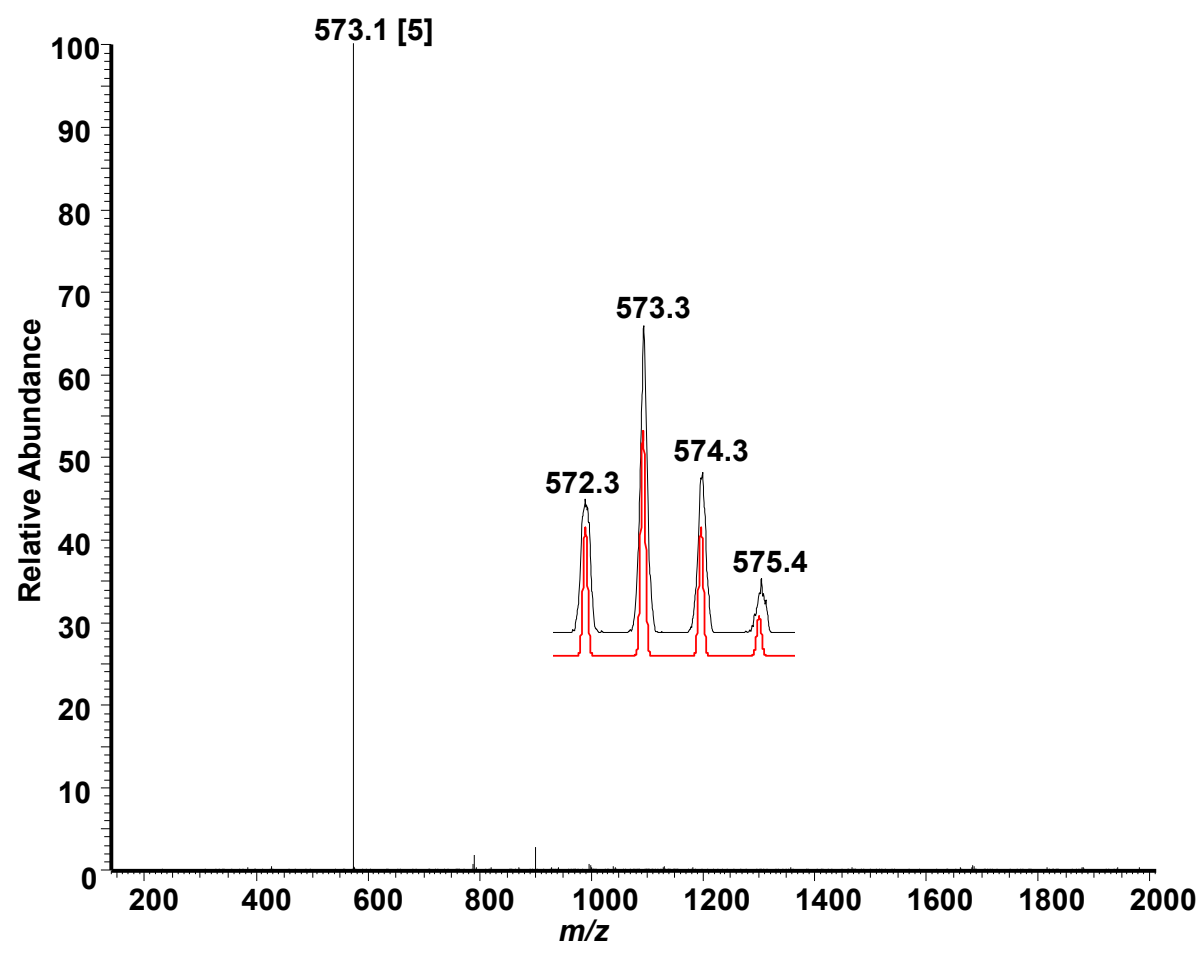

Figure S48. ESI-MS of complex $\left[\operatorname{Ag}(3)_{2}\right]^{+}$.

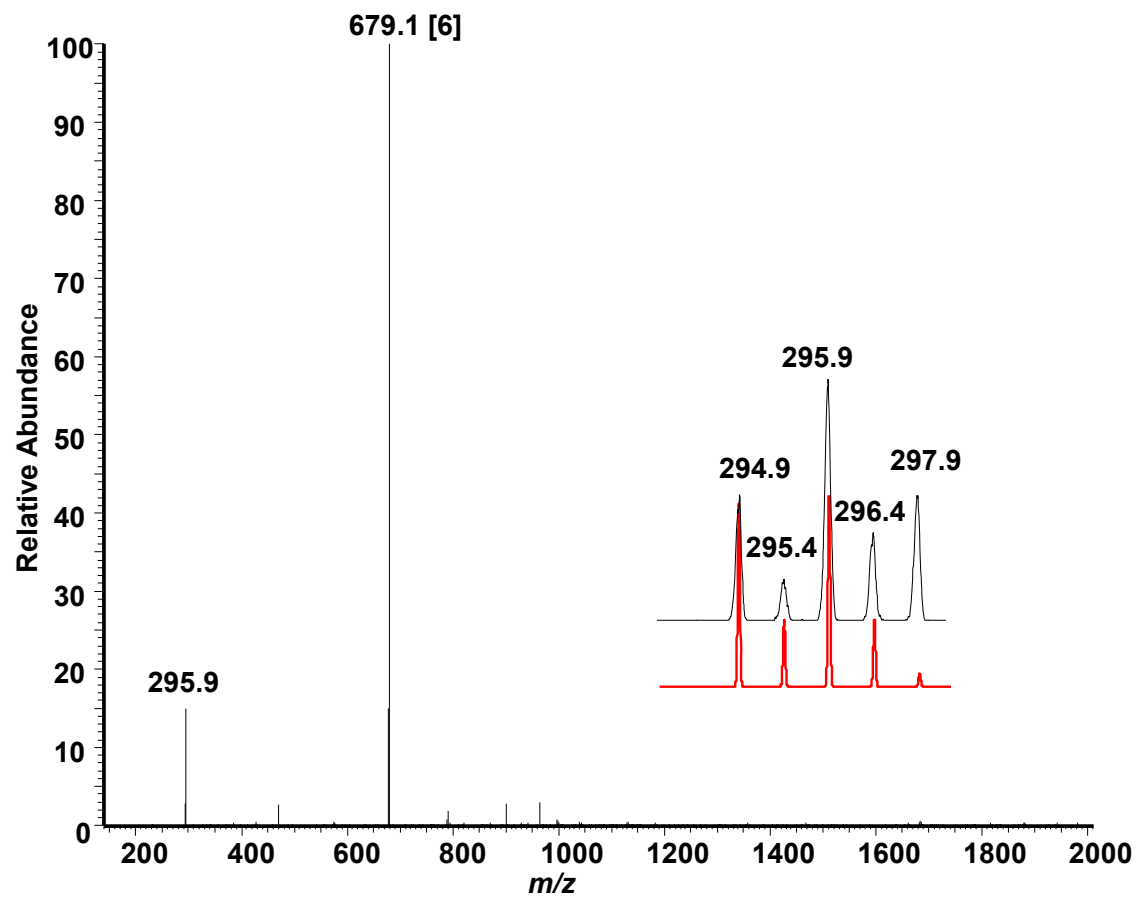

Figure S49. ESI-MS of complex $\left[\mathrm{Zn}(3)_{2}\right]^{2+}$. 


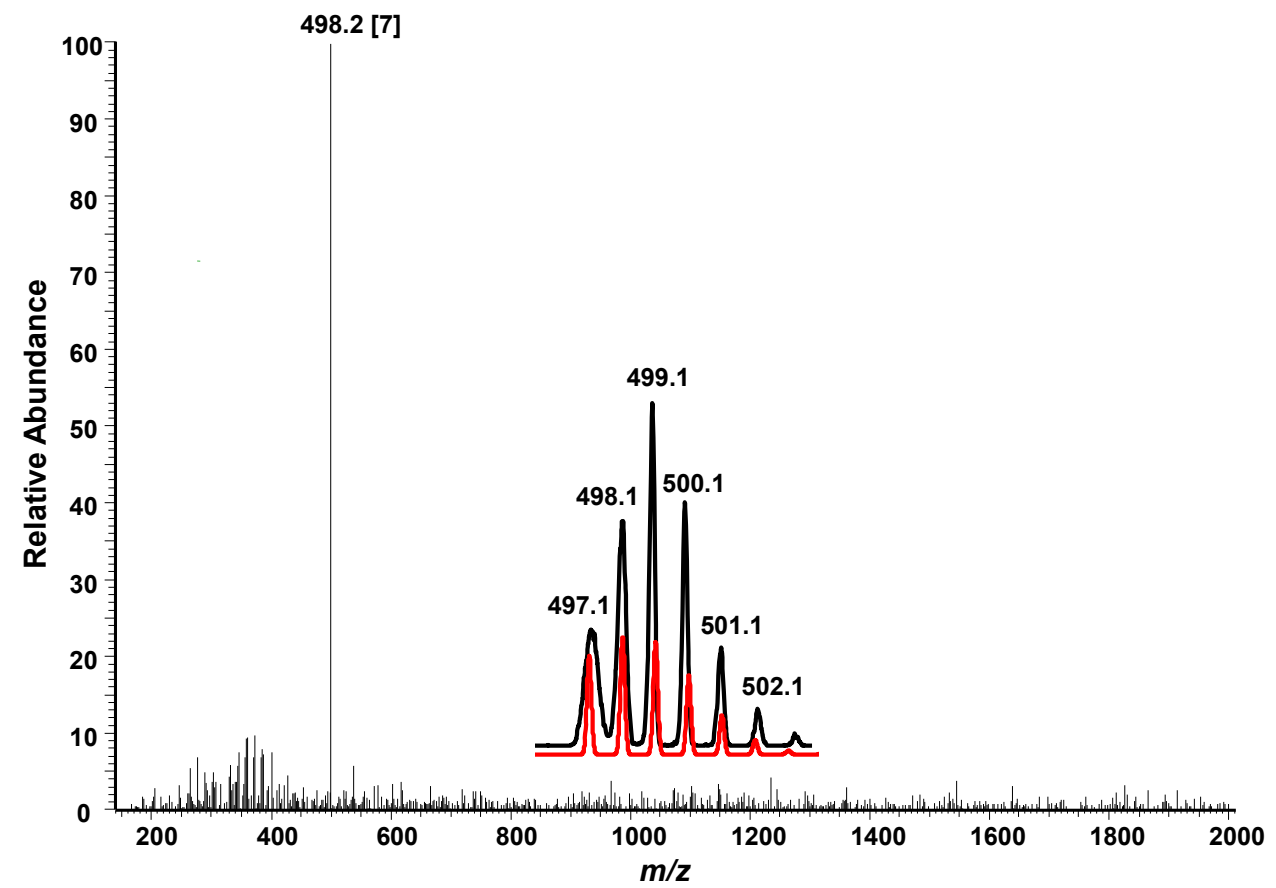

Figure S50. ESI-MS of complex $[\operatorname{Ag}(4)]^{+}$.

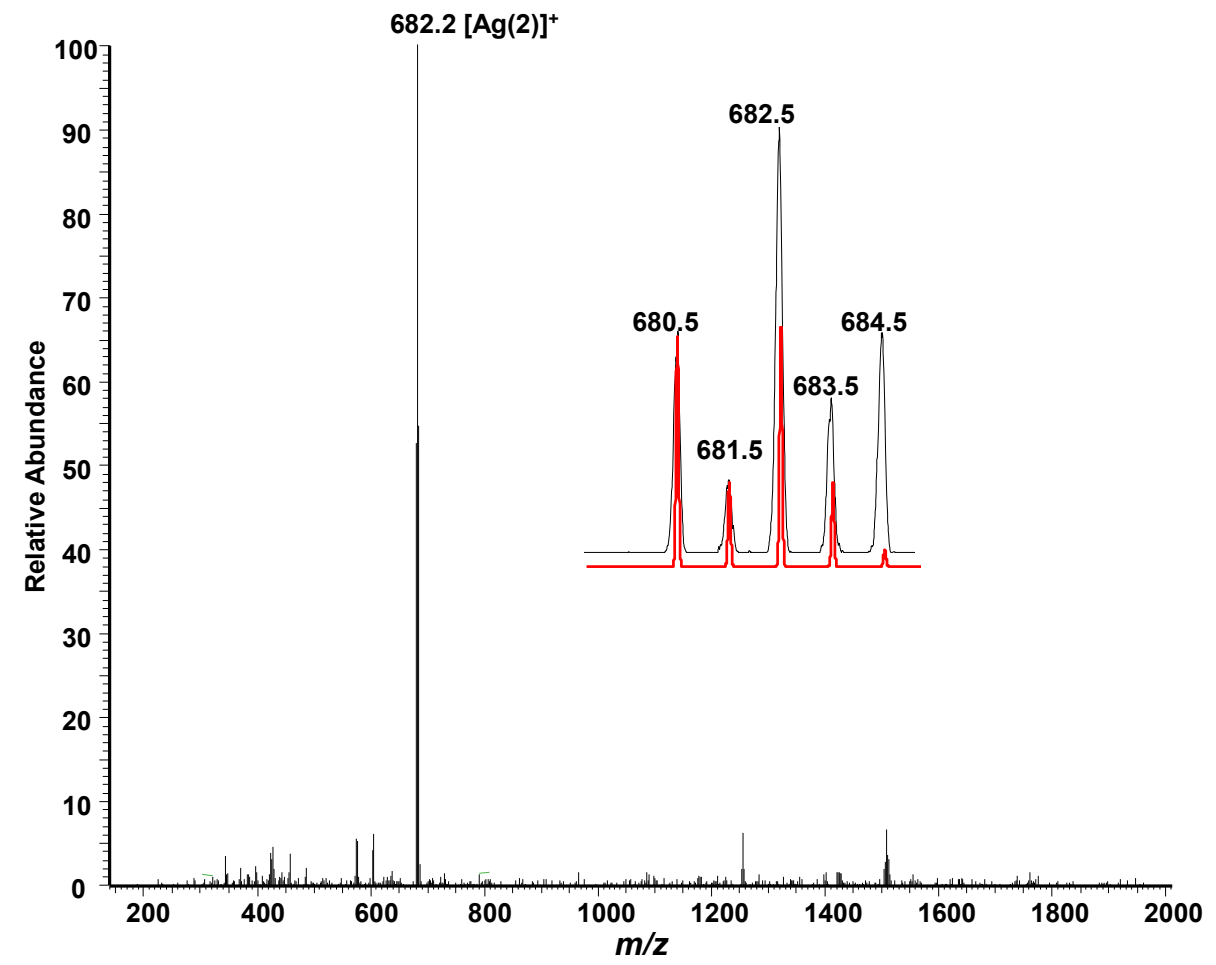

Figure S51. ESI-MS of complex $[\operatorname{Ag}(2)]^{+}$. 


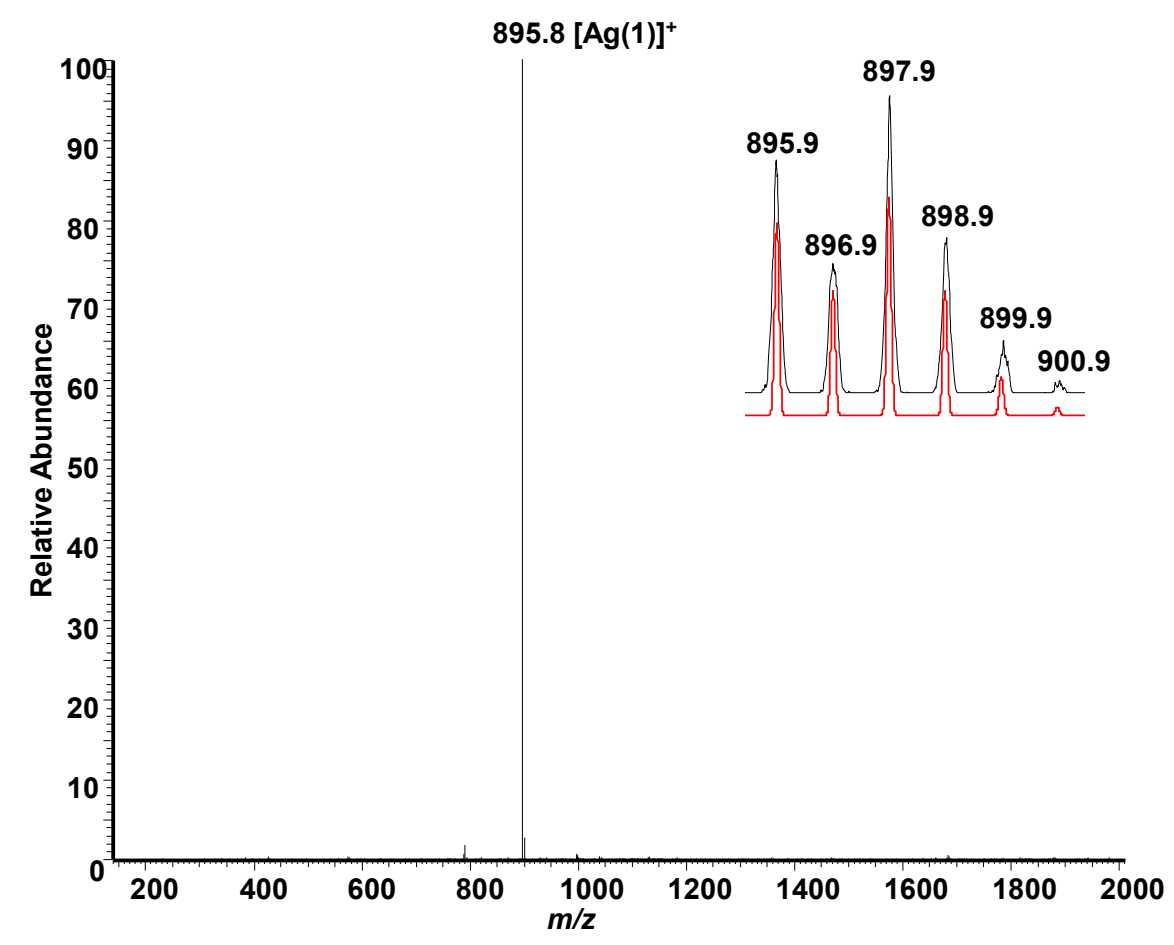

Figure S52. ESI-MS of complex $[\operatorname{Ag}(\mathbf{1})]^{+}$.

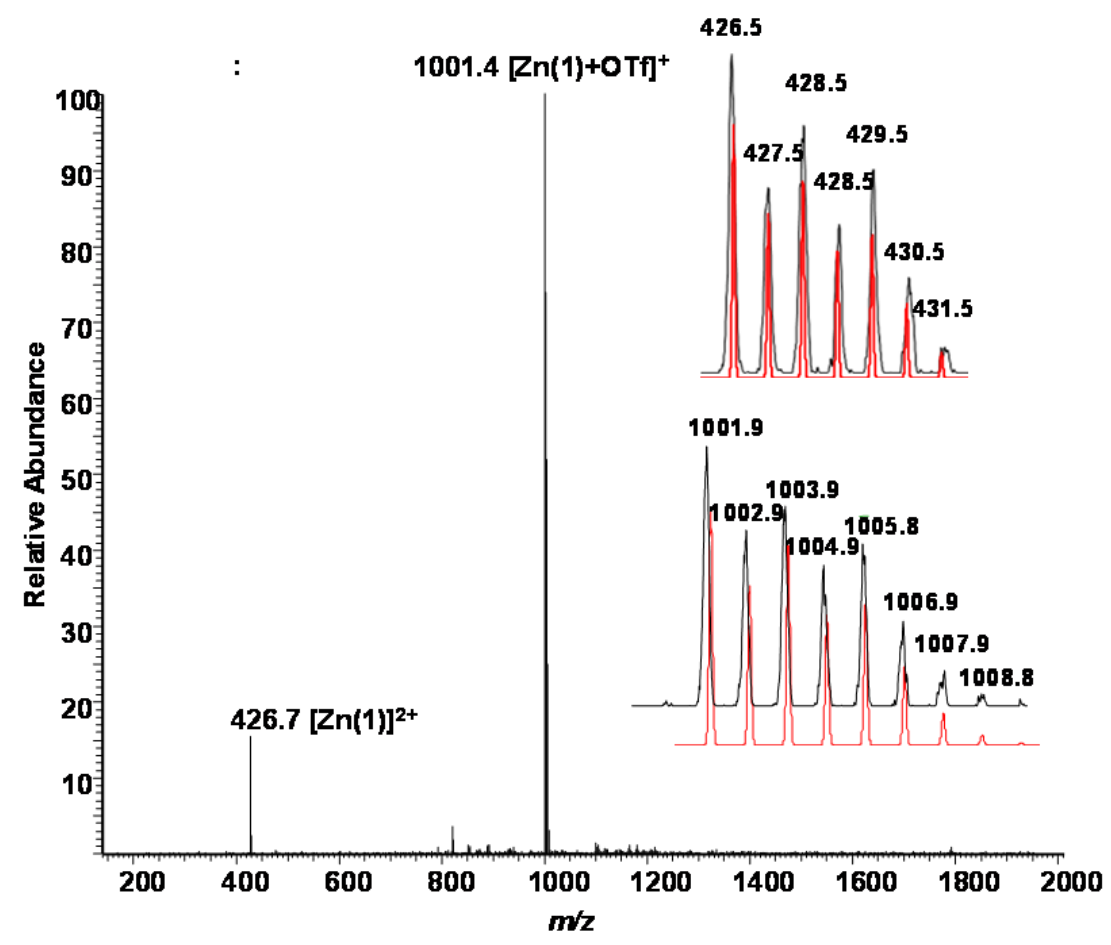

Figure S53. ESI-MS of complex $[\mathrm{Zn}(\mathbf{1})]^{2+}$. 


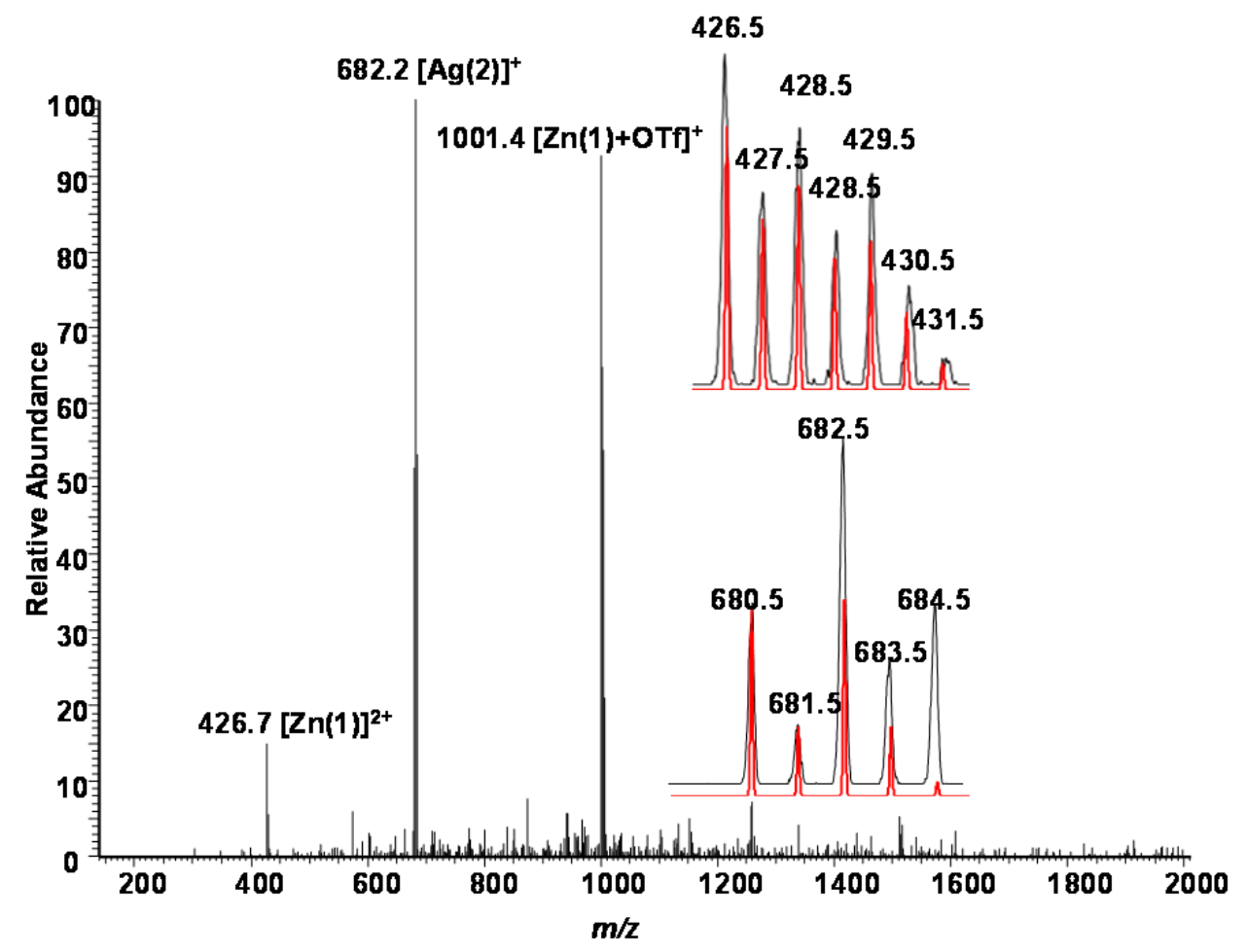

Figure S54. ESI-MS of complex NetState II. 


\section{UV-Vis and Fluorescence Data}

\section{Measurement of binding constant}

The UV-vis titration technique was used to determine the binding constants of complexes. The full data of a selected wavelength region was analyzed using SPECFIT/32 global analysis system (Spectrum Software Associates, Marlborough, MA).

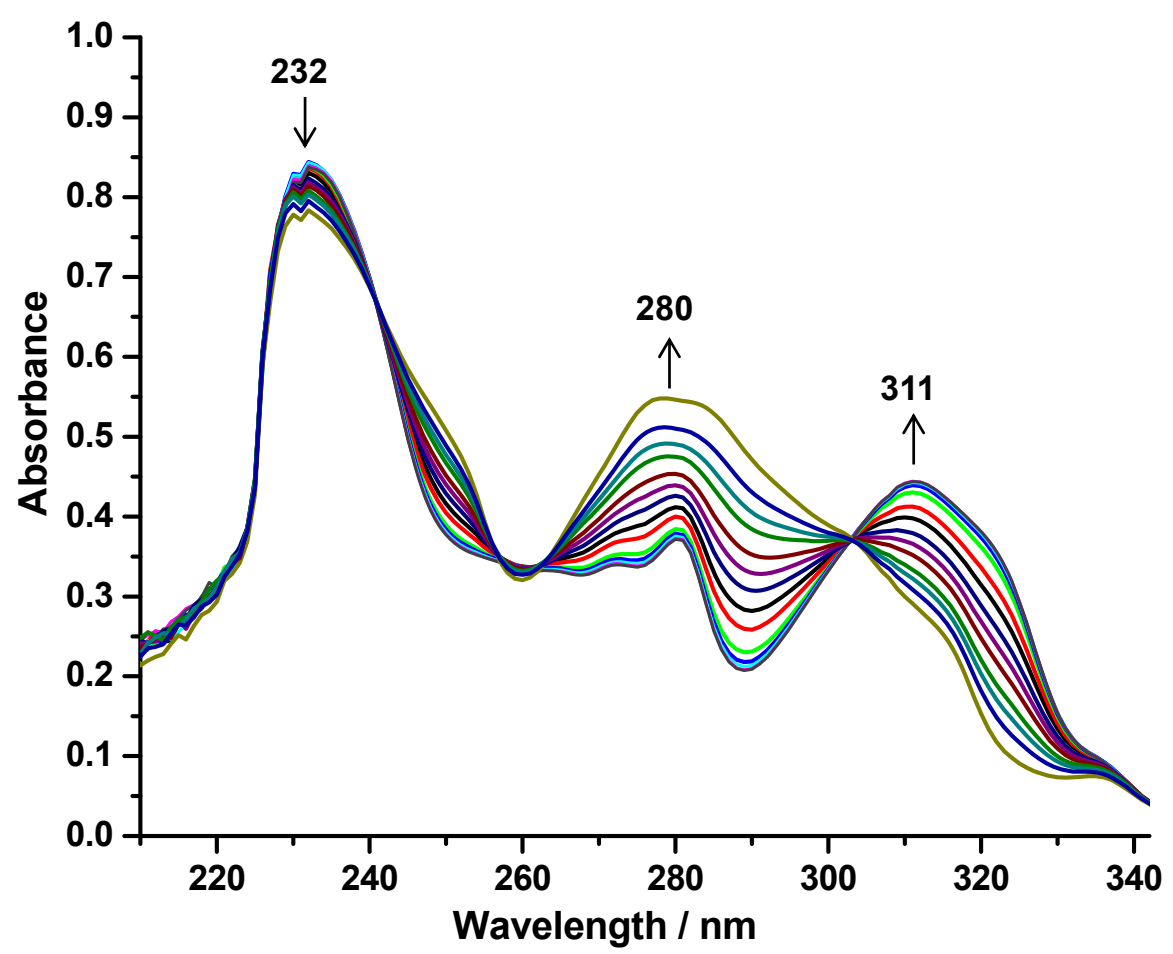

Figure S55. UV-vis spectra of $3\left(2.90 \times 10^{-5} \mathrm{M}\right)$ in $\mathrm{CH}_{2} \mathrm{Cl}_{2}(2 \mathrm{~mL})$ upon addition of $\mathrm{AgBF}_{4}\left(1.16 \times 10^{-3} \mathrm{M}\right)$ in $\mathrm{CH}_{3} \mathrm{CN}$ at $298 \mathrm{~K}$ to afford the complex $\left[\operatorname{Ag}(3)_{2}\right]^{+}$. The wavelength region 200-400 nm was analyzed. Result for $\left[\operatorname{Ag}(3)_{2}\right]^{+}: \log K=6.78 \pm 0.25$. 


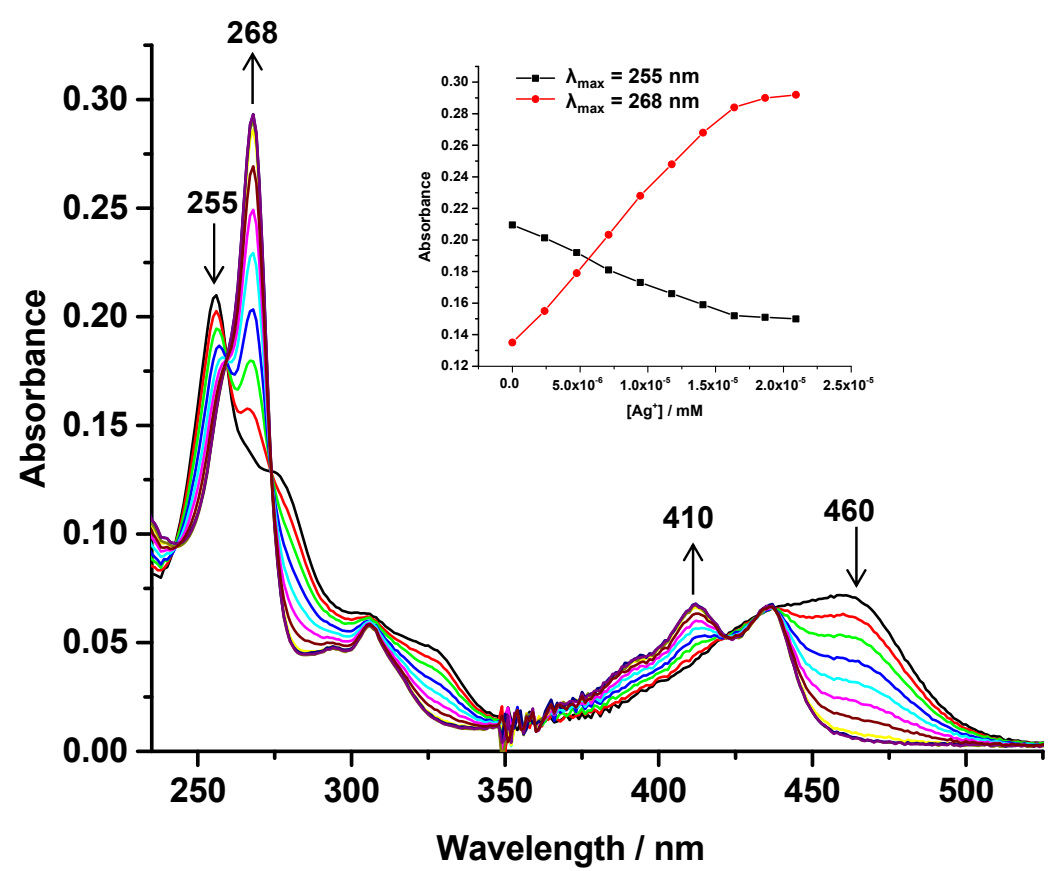

Figure S56. UV-vis spectra of $2\left(2.86 \times 10^{-6} \mathrm{M}\right)$ in $\mathrm{CH}_{2} \mathrm{Cl}_{2}(2 \mathrm{~mL})$ upon addition of $\mathrm{AgBF}_{4}\left(8.46 \times 10^{-4} \mathrm{M}\right)$ in $\mathrm{CH}_{3} \mathrm{CN}$ at $298 \mathrm{~K}$ to afford the complex $[\mathrm{Ag}(2)]^{+}$. The wavelength region $200-400 \mathrm{~nm}$ was analyzed. Result for $[\operatorname{Ag}(2)]^{+}: \log K=3.60 \pm 0.29$.

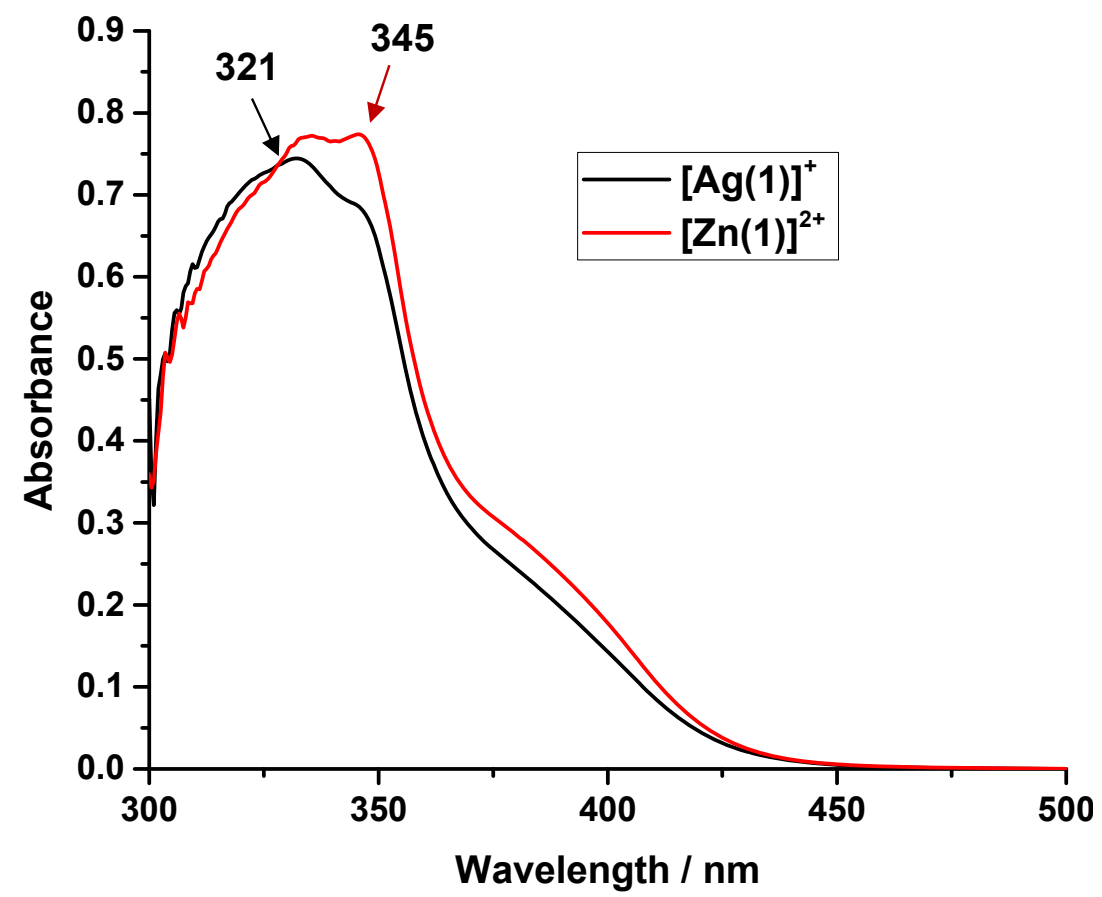

Figure S57. UV-vis spectra of complexes $[\mathrm{Ag}(\mathbf{1})]^{+}$and $[\mathrm{Zn}(\mathbf{1})]^{2+}$ in $\mathrm{CH}_{2} \mathrm{Cl}_{2}: \mathrm{CH}_{3} \mathrm{CN}=50: 1\left(10^{-5} \mathrm{M}\right)$ at $298 \mathrm{~K}$. 


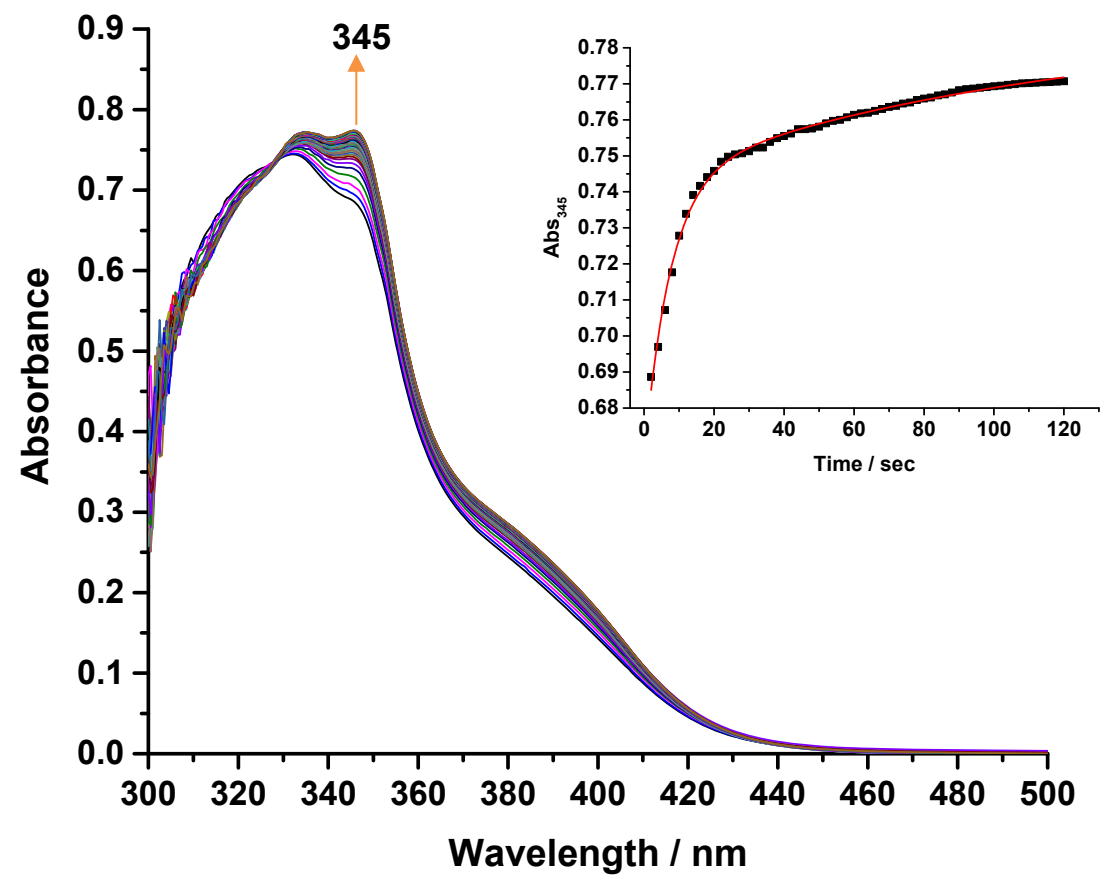

Figure S58. UV-vis spectra taken during the kinetic study of $\mathrm{Ag}(\mathbf{1})]^{+}$upon addition of $\mathrm{Zn}(\mathrm{OTf})_{2}$ to afford $[\mathrm{Zn}(\mathbf{1})]^{2+}$ in $\mathrm{CH}_{2} \mathrm{Cl}_{2}: \mathrm{CH}_{3} \mathrm{CN}=50: 1\left(10^{-5} \mathrm{M}\right)$ at $298 \mathrm{~K}$

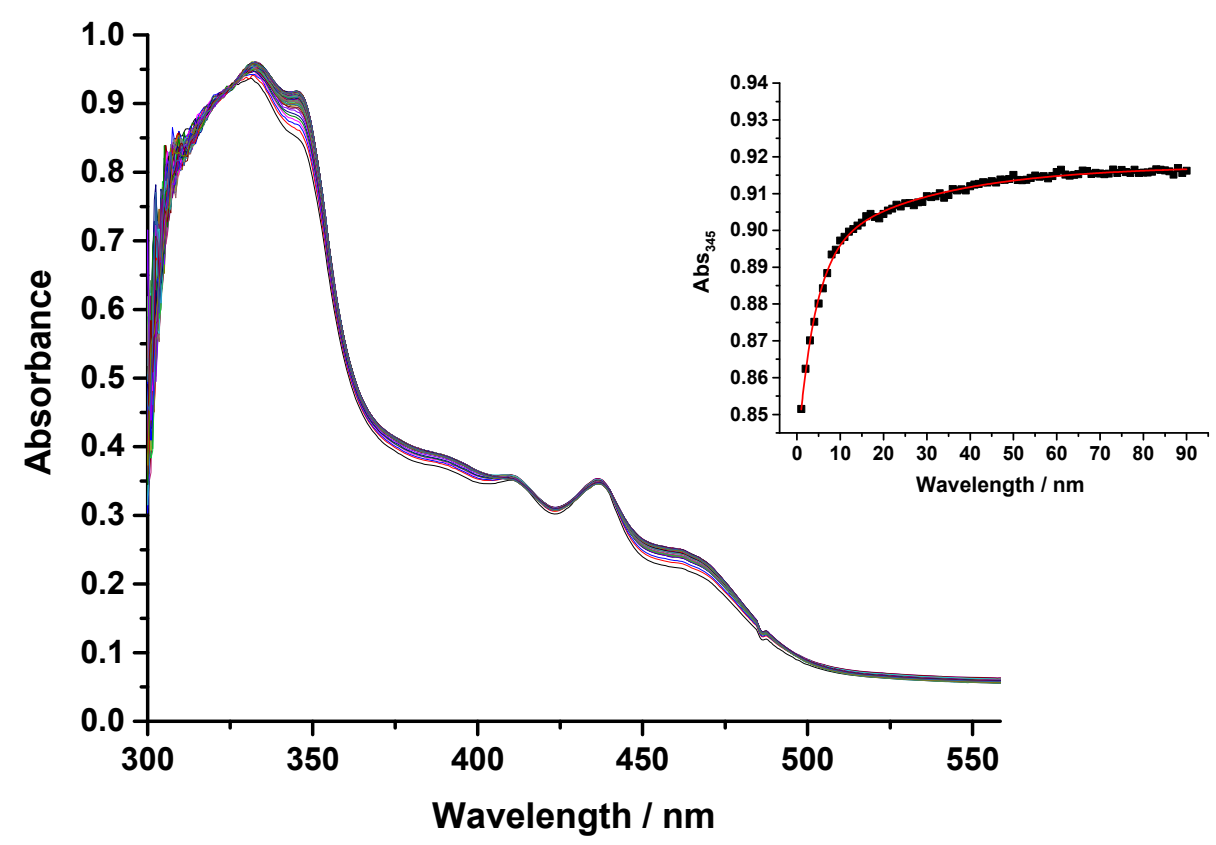

Figure S59. UV-vis spectra taken during the kinetic study of $\left([\operatorname{Ag}(\mathbf{1})]^{+}+2\right)$ upon addition of $\mathrm{Zn}(\mathrm{OTf})_{2}$ to furnish $\left([\mathrm{Zn}(\mathbf{1})]^{2+}+[\mathrm{Ag}(\mathbf{2})]^{+}\right)$in $\mathrm{CH}_{2} \mathrm{Cl}_{2}: \mathrm{CH}_{3} \mathrm{CN}=50: 1\left(10^{-5} \mathrm{M}\right)$ at $298 \mathrm{~K}$. 


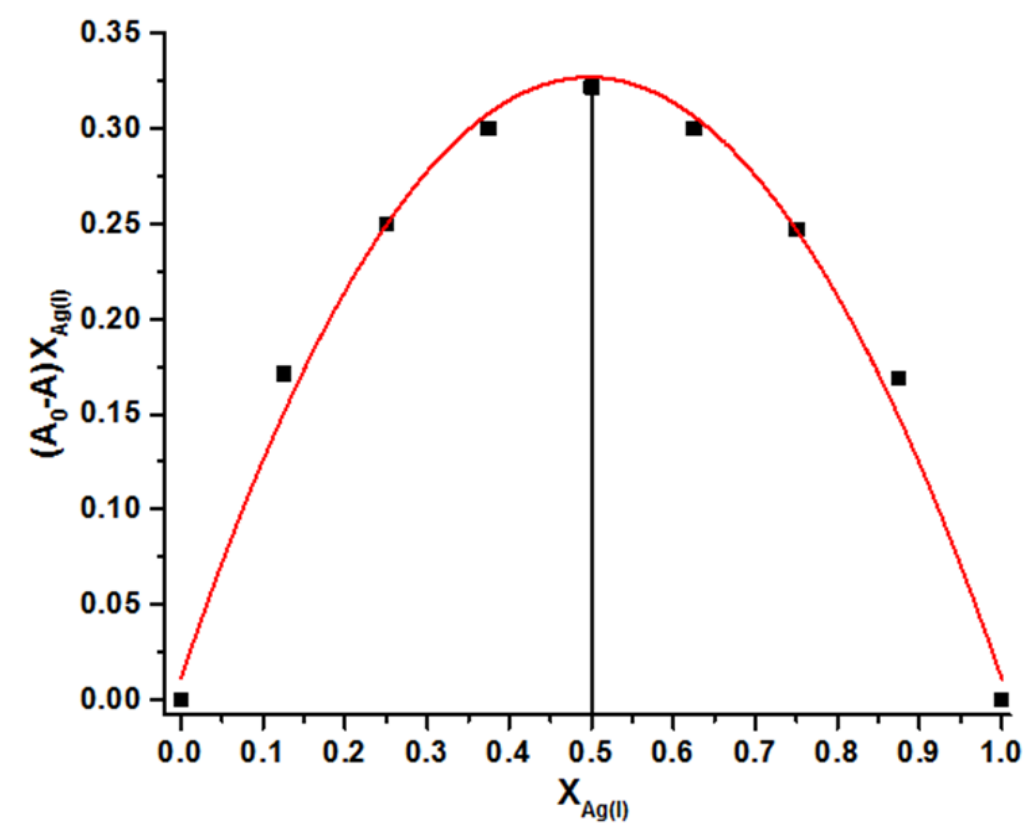

Figure S60. Job plot analysis of complex $[\operatorname{Ag}(\mathbf{2})]^{+}$using UV-vis absorption data at $\lambda=268 \mathrm{~nm}$.

(A)

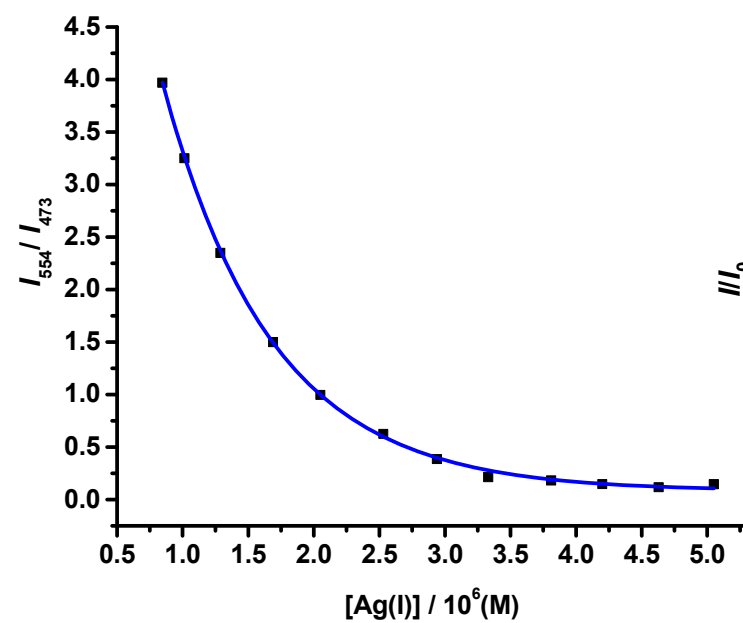

(B)

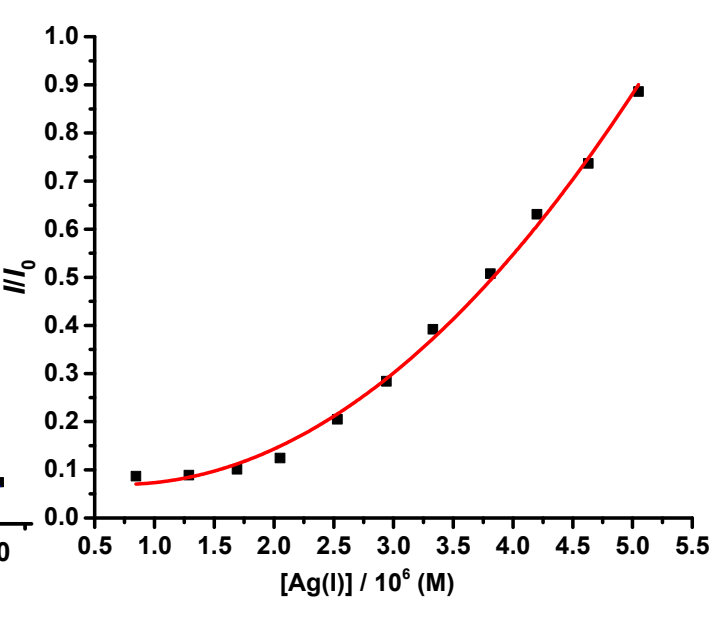

Figure S61. (A) Fluorescence intensity ratio at 554 vs $473 \mathrm{~nm}$ of probe 2 depending on the silver(I) concentration. (B) Change of fluorescence intensity at $554 \mathrm{~nm}$ of probe 2 depending on the silver(I) concentration. $I$ is the measured fluorescence intensity and $I_{0}$ is the emission intensity before the titration. 
(A)

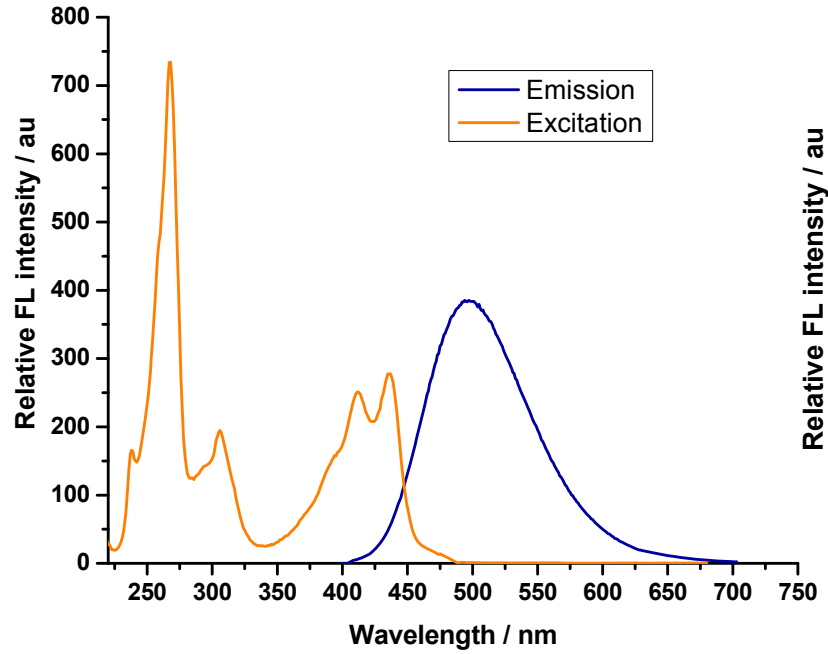

(B)

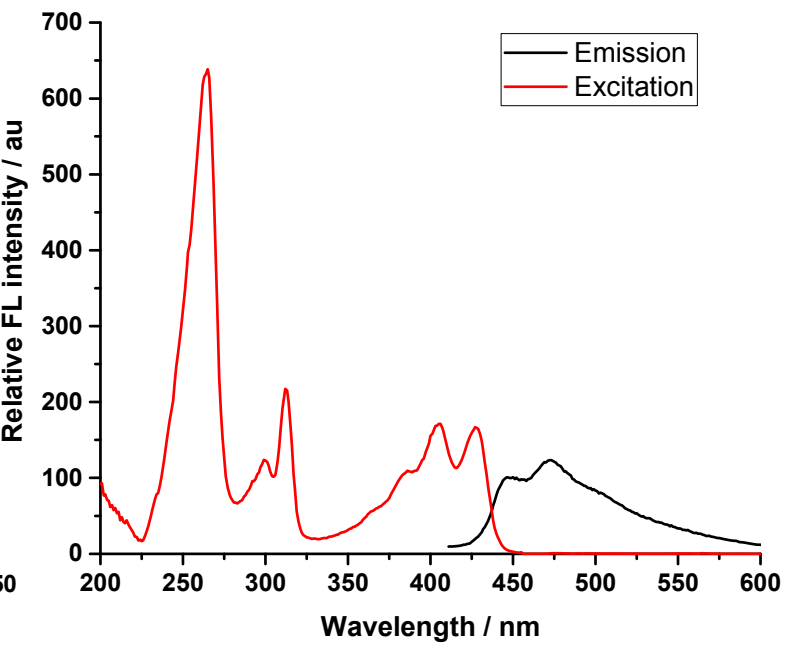

Figure S62. (A) Excitation spectra (recorded based on emission wavelength $\lambda=507 \mathrm{~nm}=$ iso-emission point of 2 and $\left.[\operatorname{Ag}(2)]^{+}\right)$and emission spectra $\left(\lambda_{\mathrm{exc}}=410 \mathrm{~nm}\right.$ ) for ligand 2. (B) Excitation spectra (recorded based on emission wavelength $\lambda=507 \mathrm{~nm}=$ iso-emission point) and emission spectra $\left(\lambda_{\mathrm{exc}}=410 \mathrm{~nm}\right)$ for complex $[\operatorname{Ag}(2)]^{+}$.

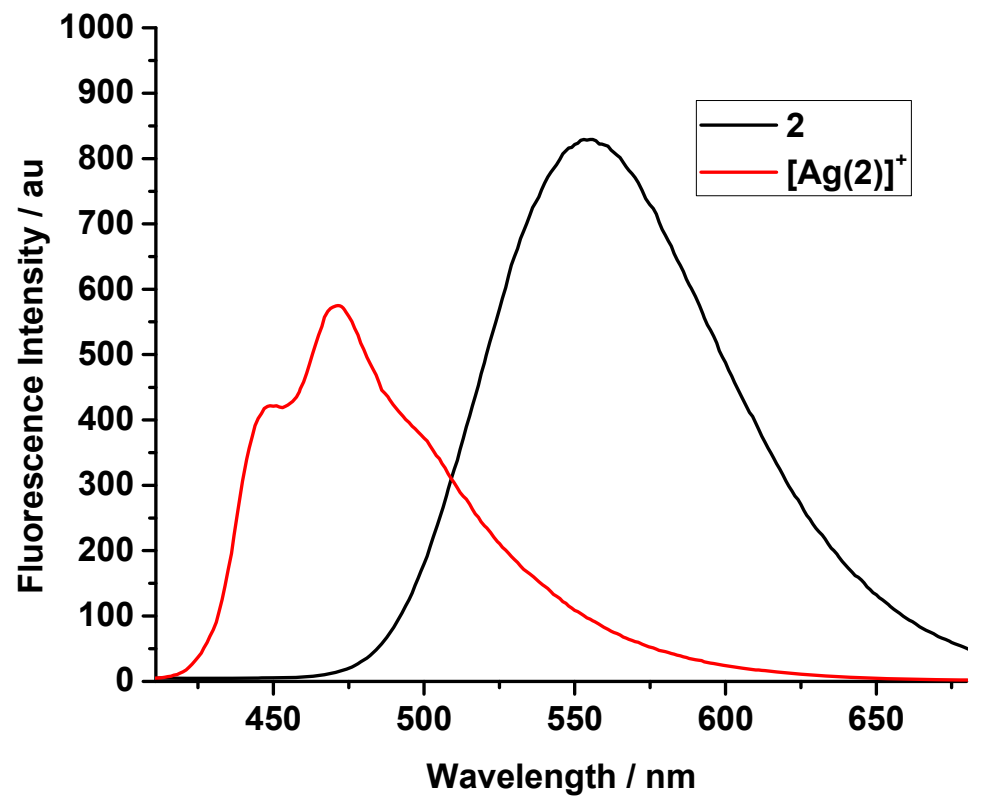

Figure S63. Emission spectra $\left(\lambda_{\mathrm{exc}}=410 \mathrm{~nm}\right)$ for ligand 2 (black) and for complex $[\operatorname{Ag}(2)]^{+}(\mathrm{red})$. 
(A)

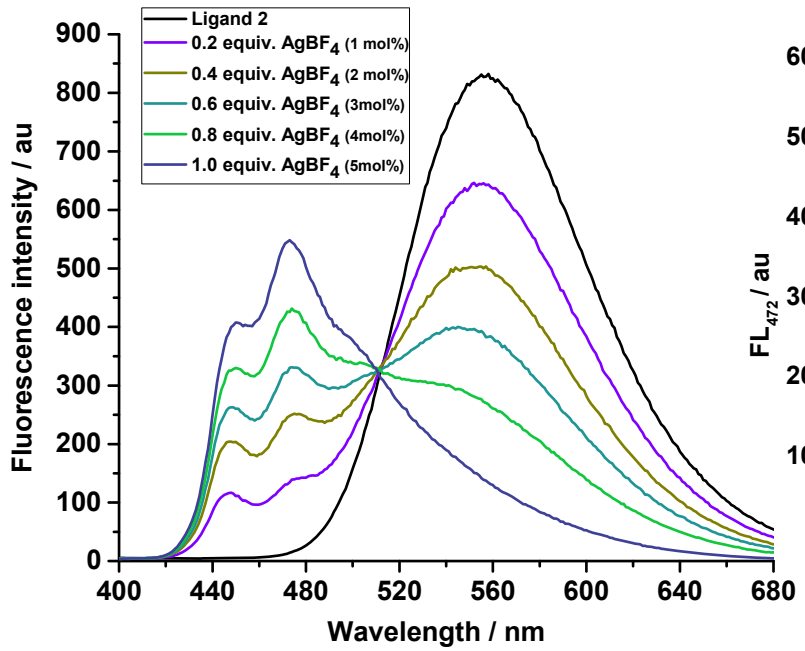

(B)

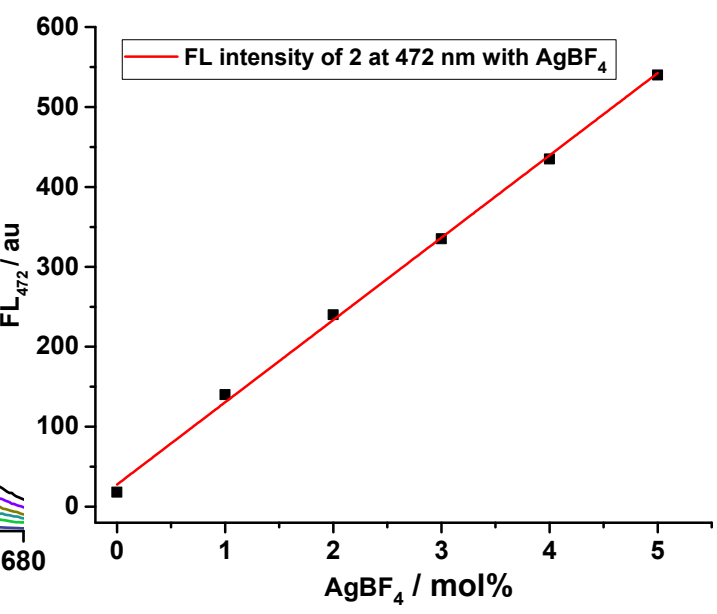

Figure S64. (A) Fluorescence titration of ligand 2 with different equivalents of $\mathrm{AgBF}_{4}(\mathrm{~mol} \%)$. (B) Change of fluorescence intensity of 2 at $\lambda=472 \mathrm{~nm}$ with the amount of $\mathrm{AgBF}_{4}$.

(A)

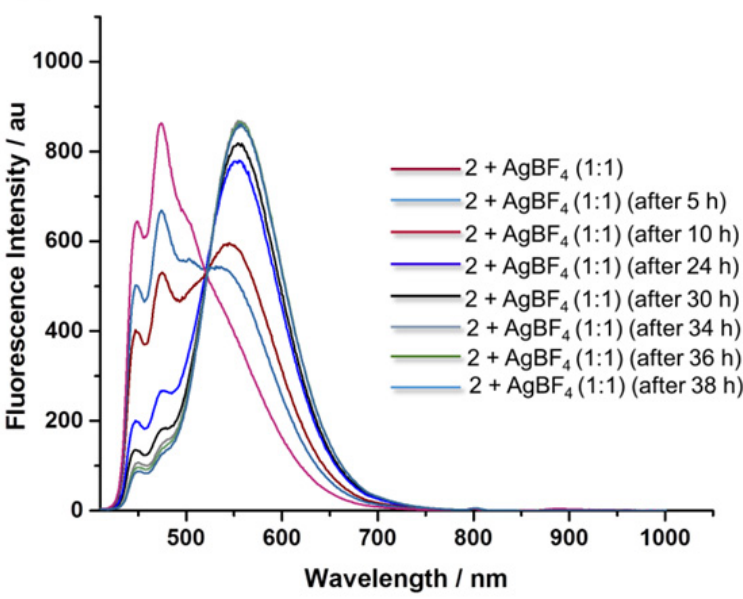

(B)

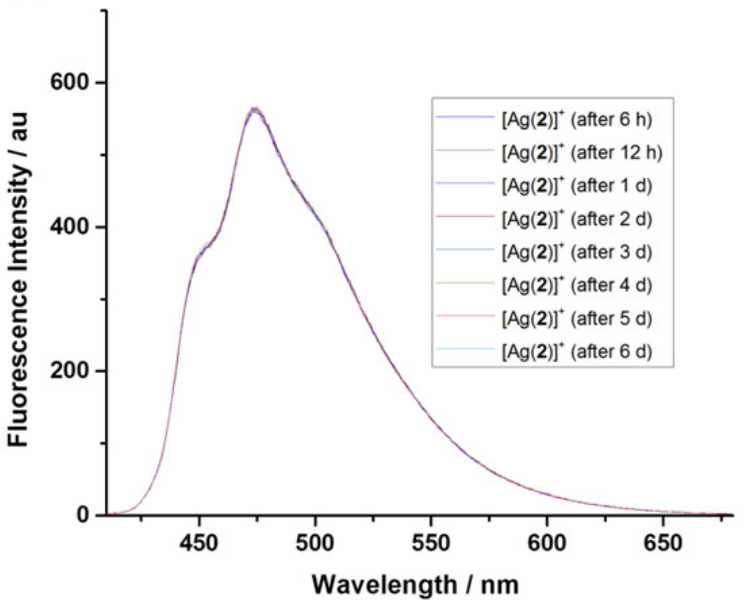

Figure S65. (A) Evaluation of the decomposition of a AgBF4 solution $\left(1.30 \times 10^{-3} \mathrm{M}\right)$ in $\mathrm{CH}_{2} \mathrm{Cl}_{2}$ with time held at ambient conditions. For the assay, an equimolar amount of $\mathbf{2}$ was given to a defined withdrawn sample of the silver(I) solution. The ratiometric response demonstrates the decomposition. (B) Change of fluorescence intensity of $[\operatorname{Ag}(2)]^{+}$solution $(2.78 \mu \mathrm{M})$ with time. In Figure S66 the changes in silver(I) content are depicted with increasing time. 


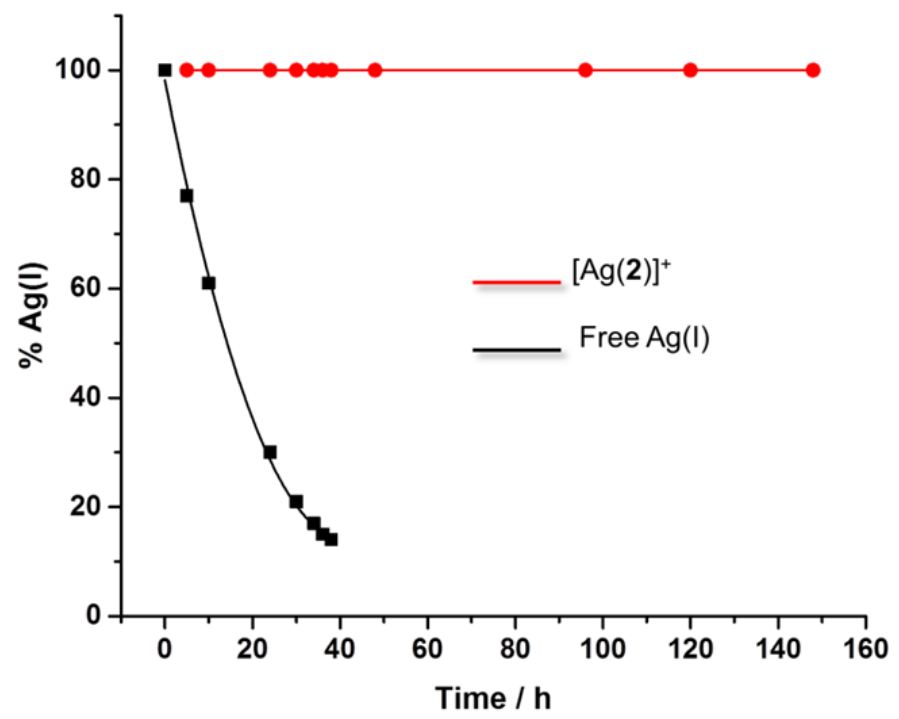

Figure S66. Plot of the changes in silver(I) content with time to document the different stability of both silver catalysts. For details see Figure S65.

\section{X-ray crystallography:}

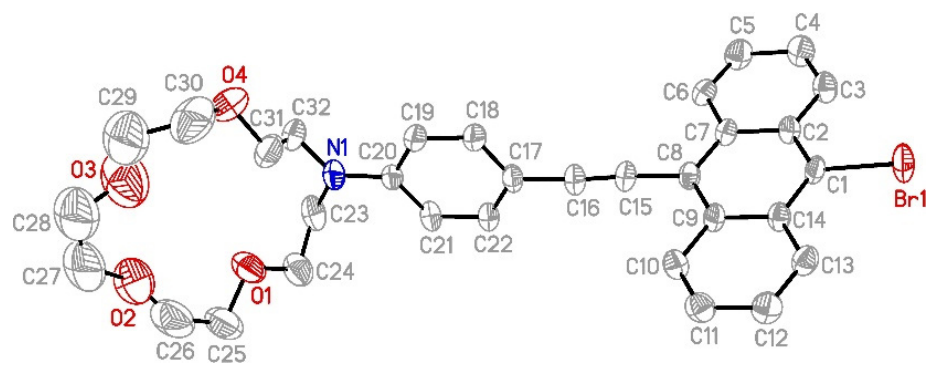

Figure S67. Perspective view of ligand 2 (X-ray structure) with thermal ellipsoids drawn at 50\% probability. Color code: C, gray; N, blue; O, red; Br, red.

Crystal structure determination. Data were collected at 293K on a STOE IPDS 2T two-circle diffractometer with a sealed tube using a graphite monochromated $\operatorname{Mo} K_{\alpha}$ radiation $(\lambda=0.71073$ $\AA$ ) and were scaled using the frame scaling procedure in the $X$-AREA program system. ${ }^{9}$ The structure was solved by direct methods using the program SHELXS ${ }^{10}$ and refined against $F^{2}$ with full-matrix least-squares techniques using the program SHELXL. ${ }^{10}$ The displacement ellipsoids of bonded atoms were restrained to have a similar shape and orientation. 


\section{Crystal data of Ligand 2}

Table 1. Crystal data and structure refinement for Ligand 2.

Identification code

Empirical formula

Formula weight

Temperature

Wavelength

Crystal system

Space group

Unit cell dimensions

Volume

$\mathrm{Z}$

Density (calculated)

Absorption coefficient

$\mathrm{F}(000)$

Crystal size

Theta range for data collection

Index ranges

Reflections collected

Independent reflections

Completeness to theta $=25.000^{\circ}$

Absorption correction

Max. and min. transmission

Refinement method

Data / restraints / parameters

Goodness-of-fit on $\mathrm{F}^{2}$

Final R indices [I $>2 \operatorname{sigma}(\mathrm{I})]$

$\mathrm{R}$ indices (all data)

Largest diff. peak and hole

CCDC deposition number
Ligand 2

$\mathrm{C}_{32} \mathrm{H}_{32} \mathrm{BrNO}_{4}$

574.49

293(2) K

$0.71073 \AA$

Triclinic

$\mathrm{P}-1$

$\mathrm{a}=9.3783(7) \AA \quad \alpha=77.106(7)^{\circ}$.

$\mathrm{b}=17.1472(15) \AA \quad \beta=86.811(7)^{\circ}$.

$\mathrm{c}=27.270(2) \AA \quad \gamma=78.249(6)^{\circ}$.

4184.9(6) $\AA^{3}$

6

$1.368 \mathrm{Mg} / \mathrm{m}^{3}$

$1.511 \mathrm{~mm}^{-1}$

1788

$0.310 \times 0.310 \times 0.110 \mathrm{~mm}^{3}$

2.172 to $25.027^{\circ}$.

$-10<=\mathrm{h}<=11,-20<=\mathrm{k}<=20,-32<=1<=32$

26203

$13985[\mathrm{R}(\mathrm{int})=0.0494]$

$94.6 \%$

Semi-empirical from equivalents

1.000 and 0.767

Full-matrix least-squares on $\mathrm{F}^{2}$

13985 / 1055 / 1027

5.085

$\mathrm{R} 1=0.1471, \mathrm{wR} 2=0.2592$

$\mathrm{R} 1=0.2206, \mathrm{wR} 2=0.2765$

2.682 and -2.798 e. $\AA^{-3}$

1888498 


\section{References}

(1) Chauvin, J.-P. R.; Pratt, D. A. Angew. Chem. Int. Ed. 2017, 56, 6255.

(2) Tomás-Mendivil, E.; Starck, J.; Ortuno, J.-C.; Michelet,V. Org. Lett. 2015, 17, 6126.

(3) Zhu, D.; Wu, Z.; Luo, B.; Du, Y.; Liu, P.; Chen, Y.; Hu, Y.; Huang, P.; Wen, S. Org. Lett. 2018, 20,4815 .

(4) Zhao, X.; Fan, W.; Miao, Z.; Chen, R. Synth. Commun. 2013, 43, 1714-1720

(5) Zhao, J.; Xu, W.; Xie, X.; Sun, N.; Li, X.; Liu, Y. Org. Lett. 2018, 20, 5461.

(6) Brachet, E.; Marzo, L.; Selkti, M.; König, B.; Belmont, P. Chem. Sci. 2016, 7, 5002.

(7) Chen, Z.; Zeng, M.; Yuan, J.; Yang, Q.; Peng, Y. Org. Lett. 2012, 14, 3588.

(8) Cheng, X.; Cao, X.; Xuan, J.; Xiao, W.-J. Org. Lett. 2018, 20, 52.

(9) Stoe \& Cie, $X$-AREA. Diffractometer control program system. Stoe \& Cie, Darmstadt, Germany, 2002.

(10) Sheldrick, G.M.; Acta Crystallogr. Sect. A, 2008, 64, 112-122. 